\title{
Development of the serotonergic cells in murine raphe nuclei and their relations with rhombomeric domains
}

\author{
Antonia Alonso · Paloma Merchán · Juan E. Sandoval • \\ Luisa Sánchez-Arrones · Angels Garcia-Cazorla $\cdot$ Rafael Artuch • \\ José L. Ferrán · Margaret Martínez-de-la-Torre $\cdot$ Luis Puelles
}

Received: 2 August 2012/Accepted: 8 September 2012/Published online: 30 September 2012

(C) The Author(s) 2012. This article is published with open access at Springerlink.com

\begin{abstract}
The raphe nuclei represent the origin of central serotonergic projections. The literature distinguishes seven nuclei grouped into rostral and caudal clusters relative to the pons. The boundaries of these nuclei have not been defined precisely enough, particularly with regard to developmental units, notably hindbrain rhombomeres. We hold that a developmental point of view considering rhombomeres may explain observed differences in connectivity and function. There are twelve rhombomeres characterized by particular genetic profiles, and each develops between one and four distinct serotonergic populations. We have studied the distribution of the
\end{abstract}

A. Alonso · J. L. Ferrán · M. Martínez-de-la-Torre .

L. Puelles $(\varangle)$

Department of Human Anatomy and Psychobiology,

Faculty of Medicine, School of Medicine,

University of Murcia, 30071 Murcia, Spain

e-mail: puelles@um.es

\section{P. Merchán}

Division of Developmental Biology, Cincinnati Children's

Hospital Medical Center, University of Cincinnati College

of Medicine, 3333 Burnet Avenue, Cincinnati, OH 45229, USA

\section{J. E. Sandoval}

Institute du Thorax, Université de Nantes, UMR,

1087 Nantes, France

L. Sánchez-Arrones

Centro de Biología Molecular Severo Ochoa, CSIC-Universidad

Autónoma de Madrid, Cantoblanco, 28049 Madrid, Spain

A. Garcia-Cazorla · R. Artuch

Neuropediatrics and Clinical Biochemistry Departments,

Hospital Sant Joan de Déu, Barcelona, Spain

A. Garcia-Cazorla · R. Artuch

Biomedical Network of Research Centres on Rare Diseases

(CIBER-ER), Instituto de Salud Carlos III, Madrid, Spain conventional seven raphe nuclei among these twelve units. To this aim, we correlated 5-HT-immunoreacted neurons with rhombomeric boundary landmarks in sagittal mouse brain sections at different developmental stages. Furthermore, we performed a partial genoarchitectonic analysis of the developing raphe nuclei, mapping all known serotonergic differentiation markers, and compared these results, jointly with others found in the literature, with our map of serotonin-containing populations, in order to examine regional variations in correspondence. Examples of regionally selective gene patterns were identified. As a result, we produced a rhombomeric classification of some 45 serotonergic populations, and suggested a corresponding modified terminology. Only a minor rostral part of the dorsal raphe nucleus lies in the midbrain. Some serotonergic neurons were found in rhombomere 4 , contrary to the conventional assumption that it lacks such neurons. We expect that our reclassification of raphe nuclei may be useful for causal analysis of their differential molecular specification, as well as for studies of differential connectivity and function.

Keywords Hindbrain - Rhombomeres - Serotonin · Midbrain $\cdot$ Pet $1 \cdot$ Lmx $1 b$

\begin{tabular}{|c|c|}
\hline \multicolumn{2}{|c|}{ Abbreviations } \\
\hline $4 \mathrm{v}$ & Fourth ventricle \\
\hline $5 \mathrm{C}$ & Motor trigeminal nucleus, caudal part \\
\hline $5 n$ & Trigeminal nerve \\
\hline $5 \mathrm{R}$ & Motor trigeminal nucleus, rostral part \\
\hline $7 \mathrm{n}$ & Facial nerve \\
\hline $8 n$ & Vestibulocochlear nerve \\
\hline $10 \mathrm{n}$ & Vagus nerve \\
\hline $11 \mathrm{n}$ & Accessory nerve \\
\hline $\mathrm{Amb}$ & Ambiguus nucleus \\
\hline
\end{tabular}




\begin{tabular}{|c|c|c|c|}
\hline $\mathrm{Cb}$ & Cerebellum & r1 & Rhombomere 1 \\
\hline CLi & Caudal linear nucleus of the raphe & r1c & Caudal part of rhombomere 1 \\
\hline CLiW & Caudal linear nucleus of the raphe, lateral wing & r1DR & r1 part of dorsal raphe nucleus \\
\hline $\mathrm{Cu}$ & Cuneate nucleus & r1DRd & r1 part of dorsal raphe nucleus, dorsal part \\
\hline DR & Dorsal raphe nucleus & r1DRv & r1 part of dorsal raphe nucleus, ventral part \\
\hline $\mathrm{DTg}$ & Dorsal tegmental nucleus & r1DRW & r1 part of dorsal raphe nucleus, lateral wing \\
\hline $\mathrm{ECu}$ & External cuneate nucleus & r1r & Rostral part of rhombomere 1 \\
\hline $\mathrm{Gu}$ & Gustatory nucleus & $\mathrm{r} 2$ & Rhombomere 2 \\
\hline $\mathrm{Gr}$ & Gracile nucleus & r3 & Rhombomere 3 \\
\hline $\mathrm{H}$ & Hindbrain & r3PnR & r3 part of pontine raphe nucleus \\
\hline ICo & Inferior colliculus & $\mathrm{r} 4$ & Rhombomere 4 \\
\hline III & Oculomotor nucleus & $\mathrm{r} 4 \mathrm{PnR}$ & $\mathrm{r} 4$ part of pontine raphe nucleus \\
\hline $\mathrm{IO}$ & Inferior olivary nucleus & r5 & Rhombomere 5 \\
\hline IPA & Interpeduncular nucleus, apical subnucleus & r5RMgD & r5 part of raphe magnus nucleus, dorsal part \\
\hline IPC & Interpeduncular nucleus, caudal subnucleus & $\mathrm{r} 5 \mathrm{RMgV}$ & r5 part of raphe magnus nucleus, ventral part \\
\hline IPPro & Interpeduncular nucleus, prodromal subnucleus & r5SGeR & r5 part of supragenual raphe nucleus \\
\hline IPR & Interpeduncular nucleus, rostral subnucleus & r6 & Rhombomere 6 \\
\hline Is & Isthmus & r6RMgD & r6 part of raphe magnus nucleus, dorsal part \\
\hline isDR & Isthmic part of dorsal raphe nucleus & r6RMgV & r6 part of raphe magnus nucleus, ventral part \\
\hline isDRd & Isthmic part of dorsal raphe nucleus, dorsal part & r6SGeR & r6 part of supragenual raphe nucleus \\
\hline isDRl & Isthmic part of dorsal raphe nucleus, lateral part & $\mathrm{r} 7$ & Rhombomere 7 \\
\hline isDRv & Isthmic part of dorsal raphe nucleus, ventral part & r8 & Rhombomere 8 \\
\hline isDRW & Isthmic part of dorsal raphe nucleus, lateral wing & r9 & Rhombomere 9 \\
\hline IV & Trochlear nucleus & r10 & Rhombomere 10 \\
\hline $\mathrm{LC}$ & Locus coeruleus & r11 & Rhombomere 11 \\
\hline LLV & Ventral nucleus of the lateral lemniscus & $\mathrm{R}$ & Red nucleus \\
\hline LRt & Lateral reticular nucleus & Rbd & Rhabdoid nucleus \\
\hline $\mathrm{LVe}$ & Lateral vestibular nucleus & $\mathrm{RMg}$ & Raphe magnus nucleus \\
\hline M & Midbrain & $\mathrm{RMgD}$ & Raphe magnus nucleus, dorsal part \\
\hline $\mathrm{m} 2$ & Mesomere 2 (preisthmic midbrain) & $\mathrm{RMgV}$ & Raphe magnus nucleus, ventral part \\
\hline $\mathrm{mDR}$ & Dorsal raphe nucleus, preisthmic & $\mathrm{ROb}$ & Raphe obscurus nucleus \\
\hline & mesencephalic part & $\mathrm{RPa}$ & Raphe pallidus nucleus \\
\hline mesV & Mesencephalic trigeminal nucleus & RtTg & Reticular tegmental nucleus of the pons \\
\hline MHB & Midbrain-hindbrain boundary & Sag & Nucleus sagulum \\
\hline mlf & Medial longitudinal fasciculus & $\mathrm{SC}$ & Spinal cord \\
\hline $\mathrm{MnR}$ & Median raphe nucleus & SCo & Superior colliculus \\
\hline MnRc & Median raphe nucleus, caudal part & SGeR & Supragenual raphe nucleus \\
\hline $\mathrm{MnRr}$ & Median raphe nucleus, rostral part & $\mathrm{SO}$ & Superior olive \\
\hline MVe & Medial vestibular nucleus & Sol & Nucleus of the solitary tract \\
\hline my & Myelomere & Sp5C & Spinal trigeminal nucleus, caudal part \\
\hline PB & Parabigeminal nucleus & Sp5I & Spinal trigeminal nucleus, interpolar part \\
\hline $\mathrm{PBr}$ & Parabrachial nucleus & $\mathrm{Sp} 5 \mathrm{O}$ & Spinal trigeminal nucleus, oral part \\
\hline PDTg & Posterodorsal tegmental nucleus & $\mathrm{SpVe}$ & Spinal vestibular nucleus \\
\hline PMB & Pontomedullary boundary & SuL & Supralemniscal raphe complex \\
\hline Pn & Pontine nuclei & $\mathrm{Tz}$ & Trapezoid body \\
\hline PnR & Pontine raphe nucleus & $\mathrm{Ve}$ & Vestibular nucleus \\
\hline $\mathrm{PO}$ & Periolivary area & VI & Abducens nucleus \\
\hline PPnR & Prepontine raphe nucleus & VII & Facial nucleus \\
\hline PPy & Parapyramidal raphe complex & $\mathrm{X}$ & Vagal dorsal motor nucleus \\
\hline Pr5C & Principal sensory trigeminal nucleus, caudal part & $\mathrm{XI}$ & Accessory nucleus \\
\hline Pr5R & Principal sensory trigeminal nucleus, rostral part & XII & Hypoglossal nucleus \\
\hline py & Pyramidal tract & xpn & Pontine decussation \\
\hline
\end{tabular}


xpy Pyramidal decussation

xscp Decussation of the superior cerebellar peduncle

xtz Trapezoid body decussation

\section{Introduction}

Serotonergic neurons associated to raphe nuclei are represented throughout the hindbrain and nowhere else in the brain (with the exception of minor midbrain and spinal additions). Nieuwenhuys (1985) reviewed literature showing that this cell type normally coexists in these nuclei with other sorts of neurons, in variable proportions. In fact, a retropontine 'nucleus raphe interpositus' is mentioned in the literature that holds no serotonergic neurons at all (Büttner-Enever et al. 1998). In the present work we concentrate on the serotonergic populations.

We now know that the hindbrain is organized developmentally in a series of transverse neuromeric units, generically named rhombomeres (Puelles et al. 2007; Nieuwenhuys et al. 2008; Nieuwenhuys 2011; Watson and Paxinos 2010). It can be deduced from known descriptions that each rhombomere probably produces a specific part of the series of raphe nuclei, but the corresponding distribution has not been determined yet. Recently, Jensen et al. (2008) used triple transgenic mappings to locate serotonergic populations derived, respectively, from $\mathrm{r} 1, \mathrm{r} 2, \mathrm{r} 3$ and $\mathrm{r} 5$, concluding that $\mathrm{r} 1-\mathrm{r} 3$ contribute more or less discretely to the classic rostral raphe nuclei (see "Discussion"). This issue is of interest, at least in order to understand the specificities observed in the projection targets and consequent possible function and pathophysiology of the individual raphe nuclei. This implies that observed differential properties derive from the singular molecular identity of their respective neuromeric origins.

Raphe nuclei were subdivided classically into rostral and caudal clusters, and given specific names (Taber et al. 1960; Lidov and Molliver 1982; Aitken and Törk 1988; Table 1). According to Dahlström and Fuxe (1964), the classic raphe nuclei fall into the following alphanumeric classification: the rostral cluster is represented by principal and caudal subdivisions of the dorsal raphe nucleus (DR/ B7 and cDR/B6), jointly with the caudal linear nucleus and the median raphe nucleus, also known as 'central superior raphe nucleus' (CLi $+\mathrm{MnR} / \mathrm{B} 8)$, the supralemniscal raphe nucleus $(\mathrm{SuL} / \mathrm{B} 9)$ and the pontine raphe nucleus (PnR/B5). The classical caudal cluster is formed by the following major groups: supragenual nucleus (SGeR/B4), nucleus raphe magnus $(\mathrm{RMg} / \mathrm{B} 3)$, nucleus raphe obscurus (ROb/B2) and nucleus raphe pallidus $(\mathrm{RPa} / \mathrm{B} 1)$, to which a group of parapyramidal serotonergic neurons can be added.

Our present aim is to advance a complete rhombomeric classification of raphe nuclei, expecting that this may help causal neuromeric analysis of shared and differential aspects of their molecular specification and differentiation. Our approach suggests a modified terminology that contemplates such developmental ascription (Table 1; Fig. 1b). In our analysis, apart of attending to literature data on genoarchitecture, fate mapping (Marín and $\mathrm{Pu}-$ elles 1995; Cambronero and Puelles 2000) and rhombomere-related lineage mapping (Jensen et al. 2008), we essentially followed the rhombomere schema of the Allen Developing Mouse Brain Atlas (http://developingmouse. brain-map.org/), whose reference atlases indicating rhombomeric units at different stages oriented our interpretation (note these reference atlases were elaborated by LP; see similar use by Watson and Paxinos 2010). Such mapping is relatively straightforward and reproducible, due to the abundance of known neuromeric landmarks. A further point of interest was to check whether the molecular profile of developing raphe populations is uniform along the diverse hindbrain neuromeric units, or shows some regional differences, irrespective of the development of a common neurotransmitter phenotype. To this end, we mapped comparatively in sagittal sections at critical developmental stages diverse gene markers previously associated to specification of the serotonergic neuronal phenotype (En1, En2, Gata2, Gata3, Lmx1b, Pet1, Slc6a4 and Tph2).

At early stages, some rhombomeric limits are identifiable as constrictions of the neural tube wall, though these flatten out as development advances and the neural wall thickens. However, there are also so-called crypto-rhombomeres in the medulla oblongata, whose interneuromeric limits are not morphologically distinguishable; these units were first found in the chick via experimental fate-mapping studies (Cambronero and Puelles 2000; in that report they were named 'pseudo-rhombomeres', but the more apt name 'crypto-rhombomeres' was thereafter suggested by R. Nieuwenhuys-personal communication to LP). The existence of crypto-rhombomeres was subsequently corroborated by the observation of corresponding molecular limits, namely step-like arrangement of the rostral borders of expression of Hox genes of the 4-8 paralogous groups, analogously to the patterns of paralogous Hox gene groups 1-3 across the overt rhombomeres (Marín et al. 2008). There are reasons to assume that the mouse medulla has the same hidden partitions (Holstege et al. 2008; Watson et al. 2010; Allen Developing Mouse Brain Atlas; Puelles 2012, in press). To corroborate our identification of interrhombomeric boundaries, we compared our 5-HT-immunoreacted or hybridized sagittal sections 
Table 1 Names and abbreviations proposed for raphe nuclei in this work, compared to classical and alpha-numeric terms

\begin{tabular}{|c|c|c|c|}
\hline $\begin{array}{l}\text { Traditional terminology and } \\
\text { classification }\end{array}$ & $\begin{array}{l}\text { Alpha-numeric } \\
\text { terminology (Dahlström } \\
\text { and Fuxe 1964; Seiger } \\
\text { and Olson 1973) }\end{array}$ & $\begin{array}{l}\text { Presently proposed } \\
\text { terminology and } \\
\text { abbreviations }\end{array}$ & Other denominations \\
\hline \multirow[t]{2}{*}{$\begin{array}{l}\text { Dorsal raphe n. (DR) (Ramón y Cajal } \\
\text { 1909; Brown 1943; Olzewski and } \\
\text { Baxter 1954; Taber et al. 1960; } \\
\text { Steinbuch and Nieuwenhuys 1985; } \\
\text { Jacobs and Azmitia 1992; Paxinos } \\
\text { and Franklin 2007; Paxinos and } \\
\text { Watson 2007) }\end{array}$} & B7 & $\begin{array}{l}\text { Dorsal raphe nn. } \\
\mathrm{mDR} \\
\text { isDRd } \\
\text { isDRl } \\
\text { isDRv } \\
\text { isDRW }\end{array}$ & \multirow[t]{2}{*}{$\begin{array}{l}\text { n. supratrochlearis (Olszewski and } \\
\text { Baxter 1954) } \\
\text { DRD/DRV/DRW-DRVL/DRL } \\
\text { (Steinbusch 1981; Diaz-Cintra } \\
\text { et al. 1981; Agnati et al. 1982; } \\
\text { Paxinos and Franklin 2007; } \\
\text { Abrams et al. 2004) }\end{array}$} \\
\hline & B6 & $\begin{array}{l}\text { r1DRd } \\
\text { r1DRv } \\
\text { r1DRW }\end{array}$ & \\
\hline $\begin{array}{l}\text { Caudal linear n. (CLi) (Castaldi 1923; } \\
\text { Steinbuch and Nieuwenhuys 1985; } \\
\text { Jacobs and Azmitia 1992) }\end{array}$ & \multirow[t]{2}{*}{ B8 } & $\begin{array}{l}\text { Caudal linear n. (Is) } \\
\text { CLi } \\
\text { Caudal linear wing } \\
\text { n. (Is) } \\
\text { CLiW }\end{array}$ & $\begin{array}{l}\text { Linear intermediate n. (Brown } \\
\text { 1943; Taber et al. 1960) } \\
\text { n. pontis oralis, pars rostralis } \\
\text { (Jacobs et al. 1984; Azmitia and } \\
\text { Gannon 1986; Hornung and } \\
\text { Fritschy 1988; Törk and } \\
\text { Hornung 1990) }\end{array}$ \\
\hline $\begin{array}{l}\text { Central superior raphe n. (CS) } \\
\text { (Bechterew 1899; Olszewski and } \\
\text { Baxter 1954; Taber et al. 1960; } \\
\text { Valverde 1962) }\end{array}$ & & $\begin{array}{l}\text { Prepontine raphe nn. } \\
\text { (r2) } \\
\text { PPnR }\end{array}$ & $\begin{array}{l}\text { Median raphe n. (Jacobs and } \\
\text { Azmitia 1992; Paxinos and } \\
\text { Franklin 2007; Paxinos and } \\
\text { Watson 2007) } \\
\text { n. linearis caudalis (Brown 1943) } \\
\text { Caudal portion of the central } \\
\text { superior raphe n. Vertes et al. } \\
1999\end{array}$ \\
\hline $\begin{array}{l}\text { Supralemniscal n. (SuL) (Törk 1990; } \\
\text { Jacobs and Azmitia 1992; Vertes } \\
\text { and Crane 1997) }\end{array}$ & B9 & $\begin{array}{l}\text { Supralemniscal } \\
\text { raphe nn. } \\
\text { r1SuL } \\
\text { r2SuL } \\
\text { r3SuL }\end{array}$ & $\begin{array}{l}\text { Caudal portion of the pontis oralis } \\
\text { n. (Törk and Hornung 1990; } \\
\text { Hornung 2003) }\end{array}$ \\
\hline $\begin{array}{l}\text { Raphe pontis n. (PnR) (Olszewski and } \\
\text { Baxter 1954; Taber et al. 1960; } \\
\text { Valverde 1962; Steinbusch 1981; } \\
\text { Törk and Hornung 1990; Harding } \\
\text { et al. 2004) }\end{array}$ & B5 & $\begin{array}{l}\text { Pontine raphe nn. } \\
\text { r3PnR } \\
\text { r4PnR }\end{array}$ & $\begin{array}{l}\text { Pontine raphe n. (Brown 1943) } \\
\text { Rostral raphe magnus n. (Taber } \\
\text { et al. 1960; Skagerberg and } \\
\text { Björklund 1985; Hornung and } \\
\text { Fritschy 1988) }\end{array}$ \\
\hline $\begin{array}{l}\text { Extraraphe cells (Olszewski and } \\
\text { Baxter 1954) }\end{array}$ & B4 & $\begin{array}{l}\text { Supragenual raphe } \\
\text { nn. } \\
\text { r5SGeR } \\
\text { r6SGeR }\end{array}$ & $\begin{array}{l}\text { Supragenual n. (Paxinos and } \\
\text { Watson 2007) }\end{array}$ \\
\hline \multirow[t]{2}{*}{$\begin{array}{l}\text { Raphe magnus n. (RMg) (Meessen } \\
\text { and Olszewski 1949; Taber et al. } \\
\text { 1960; Valverde 1962; Lidov and } \\
\text { Molliver 1982; Törk and Hornung } \\
\text { 1990; Paxinos and Franklin 2007; } \\
\text { Paxinos and Watson 2007) }\end{array}$} & \multirow[t]{2}{*}{ B3 } & $\begin{array}{l}\text { Raphe magnus nn. } \\
\text { r5RMgD } \\
\text { r6RMgD } \\
\text { r5RMgV } \\
\text { r6RMgV }\end{array}$ & $\begin{array}{l}\text { Rostral raphe obscurus n. (Taber } \\
\text { et al. 1960) } \\
\text { Central inferior raphe n. (Marburg } \\
\text { 1910) } \\
\text { Magnocellular nucleus ventralis } \\
\text { raphe (Winkler and Potter 1914) }\end{array}$ \\
\hline & & $\begin{array}{l}\text { Parapyramidal raphe } \\
\text { nn. (retropontine } \\
\text { parts) } \\
\text { r5PPy } \\
\text { r6PPy }\end{array}$ & $\begin{array}{l}\text { Lateral paragigantocell. (Jacobs } \\
\text { and Azmitia 1992) } \\
\text { Rostral ventrolateral (Törk 1990; } \\
\text { Harding et al. 2004) }\end{array}$ \\
\hline
\end{tabular}


Table 1 continued

\begin{tabular}{|c|c|c|c|}
\hline $\begin{array}{l}\text { Traditional terminology and } \\
\text { classification }\end{array}$ & $\begin{array}{l}\text { Alpha-numeric } \\
\text { terminology (Dahlström } \\
\text { and Fuxe 1964; Seiger } \\
\text { and Olson 1973) }\end{array}$ & $\begin{array}{l}\text { Presently proposed } \\
\text { terminology and } \\
\text { abbreviations }\end{array}$ & Other denominations \\
\hline \multirow{2}{*}{$\begin{array}{l}\text { Raphe obscurus n. (ROb) (Olszewski } \\
\text { and Baxter 1954; Taber et al. 1960; } \\
\text { Valverde 1962; Steinbusch and } \\
\text { Nieuwenhuys 1985; Hornung and } \\
\text { Fritschy 1988; Nieuwenhuys et al. } \\
\text { 2008) }\end{array}$} & \multirow[t]{2}{*}{ B2 } & Raphe obscurus nn. & $\begin{array}{l}\text { Posterior raphe group Ramón y } \\
\text { Cajal } 1909\end{array}$ \\
\hline & & r7-r11ROb & $\begin{array}{l}\text { Raphe parvus n. (Meessen and } \\
\text { Olszewski 1949) }\end{array}$ \\
\hline \multirow{3}{*}{$\begin{array}{l}\text { Raphe pallidus n. (RPa) (Olszewski } \\
\text { and Baxter 1954; Taber et al. 1960; } \\
\text { Valverde 1962; Steinbusch and } \\
\text { Nieuwenhuys 1985; Hornung and } \\
\text { Fritschy 1988; Nieuwenhuys et al. } \\
\text { 2008) }\end{array}$} & \multirow[t]{3}{*}{ B1 } & $\begin{array}{l}\text { Raphe pallidus nn. } \\
\text { r7-r11RPa }\end{array}$ & $\begin{array}{l}\text { Ventral nucleus. Winkler and } \\
\text { Potter (1914) }\end{array}$ \\
\hline & & $\begin{array}{l}\text { Parapyramidal raphe } \\
\text { nn. (medullary } \\
\text { parts) }\end{array}$ & $\begin{array}{l}\text { Caudal ventrolateral. (Törk1990; } \\
\text { Harding et al. 2004) }\end{array}$ \\
\hline & & r7-r11PPy & \\
\hline
\end{tabular}

with equivalent sections with mapped homeobox gene expression patterns found in the Allen Atlas database, thus correlating our data with the relevant molecular boundary landmarks (data not shown). Such expression patterns were more useful at early embryonic stages (E10.5 to E14.5), since at later stages (E16.5 to P10) many marker genes gradually downregulate their expression. However, the late developmental period is precisely when anatomical landmarks with known rhombomeric location become more distinct (nerve roots, characteristic nuclei with known neuromeric position, various decussations).

In general, the major groupings or aggregates of raphe neurons were sufficiently discrete that their neuromeric position could be resolved with reasonable reproducibility. Comparison with available literature on the connections of the raphe nuclei suggests that differential raphe projections are indeed arranged segmentally and may thus have a fundament in the differential molecular identities of the rhombomeric raphe units. Moreover, our analysis of the developmental emergence of characteristic raphe molecular typology revealed some interesting regional differences.

It has been previously reported that serotonin deficiency is a relatively common finding in neuropaediatric patients with different congenital disorders, including sudden infant death syndrome, fetal alcohol syndrome and autism (Jensen et al. 2008; De Grandis et al. 2010). However, etiological diagnosis is not achieved in most cases. This suggests that investigations of genes and histogenetic mechanisms involved in the development and maturation of functional raphe nuclei may provide in the long run new insights on the etiology of impaired serotonin transmission in the central nervous system.

\section{Materials and methods}

Animals

All mice were treated according to the stipulations and laws of the European Union (86/609/EEC) and the Spanish Government (Royal Decree 223/1998) on the care and handling of research animals. The strain used was Swiss Albino. The day of the vaginal post-coital plug formation was regarded as embryonic day 0.5 (E0.5). All procedures were performed according to protocols approved by the University of Murcia Committee for Animal Experimental Ethics. Embryos from E12.5 to E18.5 and postnatal mice from P0 to P10 were used (six embryos or mice per stage).

\section{Tissue preparation}

Embryos at E12.5 and E14.5 (stage corroborated according to Theiler 1989), were killed and fixed by immersion in phosphate-buffered $4 \%$ paraformaldehyde (0.1 M PB; pH7.4) at $4{ }^{\circ} \mathrm{C}$ for $24 \mathrm{~h}$. E18.5 embryos and postnatal animals were anesthetized on ice and perfused transcardially with PB and the fixative solution. The brains were then dissected out and postfixed for $24 \mathrm{~h}$ at $4{ }^{\circ} \mathrm{C}$.

For in situ hybridization (ISH), the brains were embedded in $4 \%$ agarose in PBS (phosphate-buffered saline solution) and sectioned $80-\mu \mathrm{m}$ thick in the sagittal or coronal planes using a vibratome (Leica Microsystems, Nussloch, Germany).

\section{Reverse-transcription polymerase chain reaction} (RT-PCR)

In order to obtain the cDNA of mouse Gata2 and Gata3 genes, we extracted RNA with Trizol reagent (Invitrogen, 
Fig. 1 Partial overview of mouse raphe nuclei in a paramedian sagittal section at $\mathrm{P} 10$. a The staining involves 5-HT-immunoreaction (brown) plus Pet1 in situ hybridization (blue). Interrhombomeric boundaries are drawn as white dashed lines (smaller dashes separate the rostral and caudal halves of r1). b Higher magnification of the periventricular area boxed in a, showing the 5-HT-positive cells of the supragenual raphe cells in r5-r6. c Schema according to a, interpreting the topological and topographic relations of the illustrated raphe nuclei within the rhombomeric map. A colorcode was applied to facilitate group distinction. Some characteristic adjacent grisea are indicated as contours for topographic reference. Note that laterally placed raphe nuclei are not shown in this figure. For abbreviations see "List of abbreviations". Scale bar $500 \mu \mathrm{m}$ in $\mathbf{a}$ and $\mathbf{c}$, and $150 \mu \mathrm{m}$ in $\mathbf{b}$

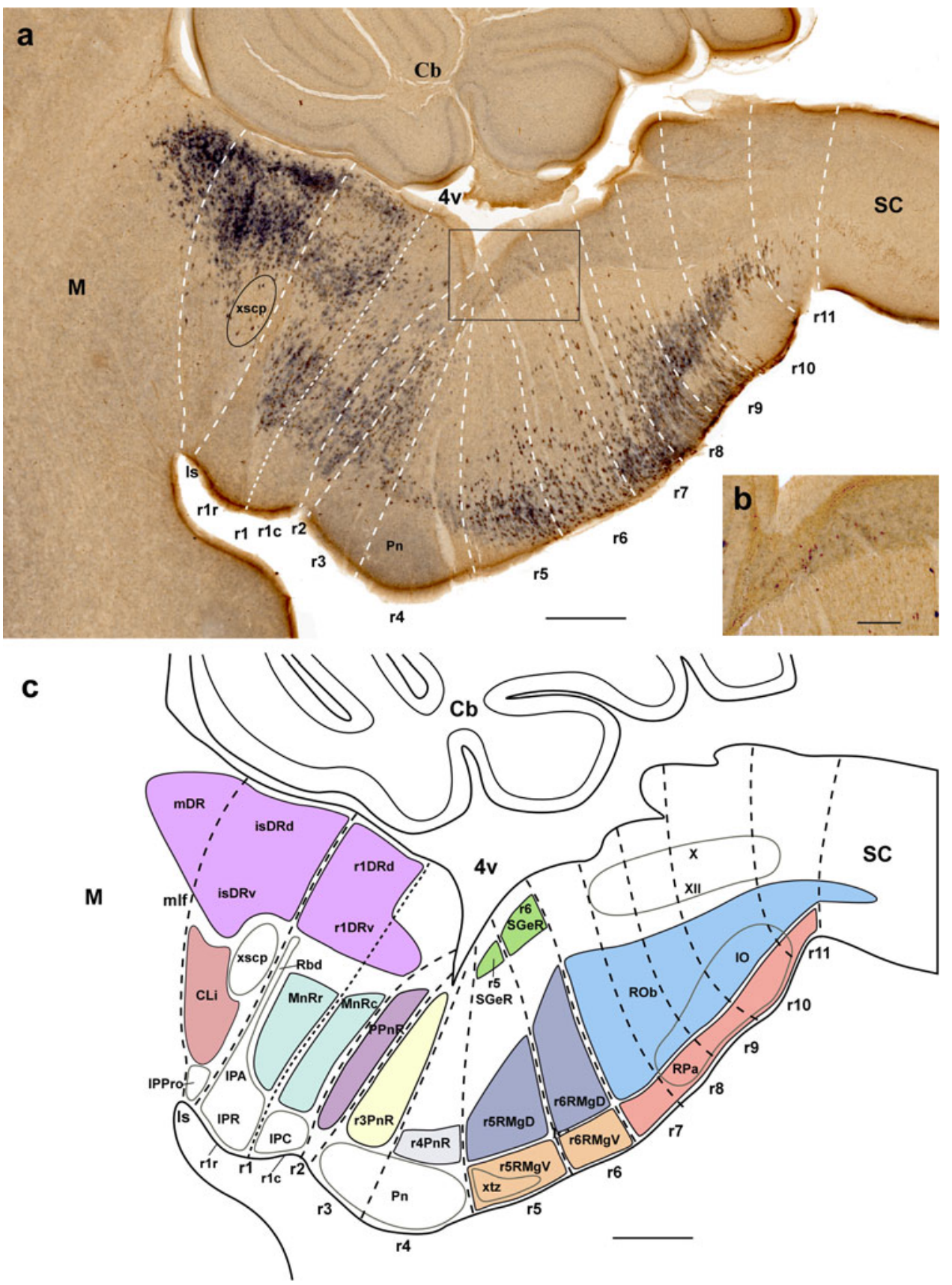

La Jolla, CA, USA) from freshly dissected mouse brains at E12.5 and E14.5 stages. The RNA was treated with DNase I (Invitrogen) for $15 \mathrm{~min}$ at room temperature, followed by enzyme inactivation at $65{ }^{\circ} \mathrm{C}$. The cDNA was obtained by reverse transcription from RNA with Superscript II reverse transcriptase and random hexamer primers (SuperScript First-Strand Synthesis System for RT-PCR; Invitrogen).

The resulting first-strand cDNA $(0.5 \mu \mathrm{L}$ of the reverse transcription reaction) was used as a template for PCR, performed with Taq polymerase (M8305, Promega,
Madison, WI) and specific primers for Gata2 (forward primer: $5^{\prime}$-cttcctccagtctctcttttgg- $3^{\prime}$, reverse primer: $5^{\prime}$-tacac cagctttggcetctg-3') and Gata3 (forward primer: $5^{\prime}$-ctgcaaa ccattaaacga- $3^{\prime}$, reverse primer: $5^{\prime}$-acgtctccagcttcatgctatc) mRNAs. PCR conditions were as follows: $5 \mathrm{~min}$ at $94{ }^{\circ} \mathrm{C}$, then 35 cycles $\left(30 \mathrm{~s}\right.$ at $94{ }^{\circ} \mathrm{C}$, plus $1 \mathrm{~min}$ at $T_{\mathrm{m}}$ temperature, $58{ }^{\circ} \mathrm{C}$, and $1 \mathrm{~min}$ at $72{ }^{\circ} \mathrm{C}$ ), followed by $10 \mathrm{~min}$ at $72{ }^{\circ} \mathrm{C}$. The PCR products were cloned into pGEM-T Easy Vector (Promega) and sequenced (SAI, University of Murcia). 
In situ hybridization

The Enl (NM010133.2, positions 147-2032), En2 (NM 010134.3, positions 1316-2098), Lmxlb (NM 010725.1, positions 1-898), Otx2 (NM 144841.2, positions 592-1165) and Petl (NM 153111.1, positions 897-1396) riboprobes were synthesized from plasmids kindly provided by $\mathrm{K}$. Schughart (En1), J. Rossant (En2), R. Johnson (Lmxlb), A. Simeone (Otx2) and W. Wurst (Pet1). The riboprobes Tph2 and Slc6a4 were supplied by ImaGenes (Berlin, Germany) from their Mouse EST collection (Tph2, clone RZPDp981F09257D, NM 173391.1, positions 47-649; Slc6a4, clone RZPDp981H09201D, NM 010484.1, positions 59-389). The Gata2 (NM 008090, positions 1680-2441) and Gata3 (NM 008091.2, positions 68-785) riboprobes were synthesized from cDNA cloned at our laboratory. All cDNA used in this work was sequenced (SAI, University of Murcia) and specificity was checked using the BLAST tool (NCBI).

The hybridizations on floating vibratome-sections were done according to the protocol of Shimamura et al. (1994). As general in situ hybridization (ISH) controls, sense and antisense probes were applied to adjacent representative sections (the signal was present only with antisense probe), and some sections were processed without either sense or antisense probes, to check for possible background due to the other reactives used in the standard ISH procedure. To detect the hybridized product, the sections were incubated overnight with alkaline phosphatase-conjugated antidigoxigenin Fab fragments (1:3,500, Roche Diagnostics, Manheim, Germany), and nitroblue tetrazolium/bromochloroindolyl phosphate (NBT/BCIP) was used as chromogenic substrate for the final alkaline phosphatase reaction (Boehringer, Mannheim, Germany).

\section{Immunohistochemistry}

All immunoreacted sections were processed following the same free-floating protocol, including those processed after ISH. Sections were washed in PBS and then treated with $0.1 \%$ hydrogen peroxide in PBS for $1 \mathrm{~h}$ in the dark to inactivate endogenous peroxidase activity. After several rinses in PBT (PBS with $0.2 \%$ Triton X-100), sections were blocked with $0.5 \%$ goat serum, $0.2 \%$ bovine serum albumin (BSA) and $0.2 \%$ Triton X-100 (Sigma, St. Louis, MO, USA) in PBS for $4 \mathrm{~h}$, and then, incubated overnight at $4{ }^{\circ} \mathrm{C}$ with polyclonal rabbit anti-5-HT antibody $(1: 1,000$; ImmunoStar, Hudson, USA; cat. no 20080), prepared in the same blocking solution. This primary reaction was developed with biotinylated goat anti-rabbit secondary antibody (1:200, $2 \mathrm{~h}$ of incubation; Vector Laboratories, Burlin- game, CA, USA), and then with streptavidin/horseradish peroxidase (HRP) complex (1:200, $2 \mathrm{~h}$ of incubation; Vectastain-ABC kit; Vector Laboratories, Burlingame, CA, USA). All antibodies were diluted in the same blocking solution as the primary antibody. The histochemical detection of the peroxidase activity was carried out using $0.03 \%$ diaminobenzidine $(\mathrm{DAB})$ and $0.005 \% \mathrm{H}_{2} \mathrm{O}_{2}$. After immunoreaction, the sections were mounted, dehydrated and then coverslipped with Eukitt (Fluka, Buchs, Switzerland).

Antisera characterization

The commercial rabbit anti-5-HT polyclonal antibody employed in this report was raised against serotonin derived from rat brain, coupled to bovine serum albumin (BSA) with paraformaldehyde (Immunostar, Hudson, WI, USA; catalog number 20080; manufacturer's technical information). This antibody was shown to recognize specifically serotonin molecules (5-hydroxytryptamine) in mouse brainstem (Fortune and Lurie 2009). The 5-HT immunoreactions obtained in our mouse brainstem tissue reveal a virtually identical staining pattern as the Petl riboprobe, which is a specific marker of the serotonergic neuronal phenotype (Hendricks et al. 1999). Moreover, pre-adsorption of the diluted antibody with $25 \mu \mathrm{g} / \mathrm{mL}$ of serotonin/BSA complex eliminated completely the reaction, whereas pretreatment with BSA did not affect the immunostaining.

Imaging

Digital microphotographs were obtained with a Zeiss Axiocam camera (Carl Zeiss, Oberkochen, Germany) or with a ScanScope digital slide scanner (Aperio, Vista, CA, USA) and the images were corrected for contrast and brightness using Photoshop CS3 (Adobe Systems, San Jose, CA, USA). All plates were produced and labeled in Adobe Illustrator CS3 software (Adobe Systems, San Jose, CA, USA).

\section{Results}

Generalities

We first addressed serotonergic cell distribution at P0 and $\mathrm{P} 10$, when the morphology of raphe nuclei is essentially definitive (Figs. 1, 2, 3; Watson and Paxinos 2010). The traditional mammalian raphe nuclei (Taber et al. 1960, and other authors; see Table 1), or B1 to B9 groups of Dahlström and Fuxe (1964), were assigned tentatively to indi- 
vidual rhombomere-derived territories. A small rostral part of the dorsal raphe nucleus maps within the caudal midbrain (Fig. 1); see comments on this below.

According to the schema of rhombomeres employed in the Allen Developing Mouse Brain Atlas, which is based on the experimental fate-mapping or Hox-gene-mapping works of Marín and Puelles (1995), Cambronero and Puelles (2000), Marín et al. (2008), and Holstege et al. (2008), we contemplate 12 rhombomeric units, ranging from the isthmus (r0) to $\mathrm{r} 11$, next to the rhombo-spinal junction. Note there are authors that alternatively encompass our r8-r11 units within a large ' $r 8$ ' rhombomere, and also lump our Is-r1 units into an extra-large ' $r 1$ ' domain, due to variant criteria about how to define a rhombomere (Lumsden and Krumlauf 1996; compare Puelles and Rubenstein 2003; Puelles 2009a, b); this controversy is irrelevant for our present purpose, since readers can lump our conclusions according to their own rhombomeric schema. We agree with Vaage (1969, 1973) in distinguishing rostral and caudal parts of $r 1$ (r1r, r1c; Fig. 1). Both Puelles et al. (2007) and the Allen Developing Mouse Brain Atlas propose prepontine (Is, $\mathrm{r} 1-\mathrm{r} 2)$, pontine (r3-r4), pontomedullary or retropontine (r5-r6) and medullary (r7-r11) hindbrain subregions. Raphe nuclei have been subdivided classically into rostral and caudal clusters (Lidov and Molliver 1982; Aitken and Törk 1988), which correspond to blocks developing within Is-r4 and r5-r11, respectively. It is often stated in the literature that $\mathrm{r} 4$ lacks altogether a serotonergic raphe population (e.g., Pattyn et al. 2003). However, we do observe a small population at this locus (e.g., Fig. 1).

In Fig. 1, we mapped serotonin immunoreaction jointly with ISH for the gene Petl, a serotonergic marker, at P10. Visibly, postnatal serotonergic neurons are not grouped uniformly along the rostro-caudal series of rhombomeres. In some rhombomeres (e.g., r4) there is only a sparse raphe population, whereas in others we find several separate dense aggregates (e.g., in r1). These are described in detail in the next section. The serotonergic nuclei mainly occupy the paramedian basal region of the rhombomere they belong to (only occasionally penetrating secondarily the median raphe region proper, which is a cell-poor, floorplate-derived, astroglial palisade). Individual raphe populations differ in their radial position (stratification) within the paramedian basal plate, occupying positions at either the periventricular, intermediate or superficial strata. In addition, there is also some mediolateral dispersion. Some serotonergic elements appear displaced laterally from the raphe neighborhood, at periventricular, intermediate or superficial positions, but nevertheless remain always within the basal plate.
Fig. 2 Pet 1 expression detected by in situ hybridization across the midbrain-hindbrain continuum, shown in a series of 14 nonconsecutive standard 'cross sections' at P10 (these are first horizontal and then transversal to the rhombomeres; see schema of section levels and planes in $\mathbf{o}$, based on Fig. 1c). The rhombomeric boundaries are traced as thin dashed lines. Selected other structures are identified for topographic reference. a-c Levels through rostral midbrain and ventral parts of prepontine/pontine hindbrain (sections are here distinctly horizontal). d-f Levels through caudal midbrain, dorsal parts of prepontine/pontine hindbrain and rostral cerebellum (sections are still largely horizontal). g-n Levels through retropontine and medullary hindbrain (transversal sections). o Schema representing the different levels of section shown in a-n. Black stars in $\mathbf{h}-\mathbf{m}$ mark subpial pet1-positive cells found superficial to the pyramidal tract (py). Arrowheads in $\mathbf{m}$ and $\mathbf{n}$ point out petl-positive cells associated to the pyramidal decussation (xpy). For abbreviations see "List of abbreviations". scale bar $350 \mu \mathrm{m}$ in a-n, and $150 \mu \mathrm{m}$ in $\mathbf{o}$

Rhombomeric pattern of mouse raphe nuclei at $\mathrm{P} 0$ and P10

\section{The rostral cluster}

This cluster is characterized by a stereotyped distribution of its elements in the periventricular, intermediate and superficial strata, largely within the four rostralmost rhombomeres (Is, r1-r3), to which must be added the mentioned minor population in $\mathrm{r} 4$ and the small subpopulation found within the periaqueductal gray of the caudal midbrain (Figs. 1, 2a-f, 3). We will deal separately with the latter.

Isthmic raphe nuclei The present identification of these elements rests upon the capacity to identify the isthmic territory of the brainstem as distinct from the midbrain (see Puelles et al. 2012b) and rhombomere 1 (note that several authors assume that the isthmus is merely a vaguely defined rostral part of $\mathrm{r} 1$; see "Discussion"). We used Otx2 expression selectively present in the midbrain to identify the rostral boundary of the Is (Simeone et al. 1992, 1994). The caudal isthmic limit was estimated by the selective presence of numerous Pax7, Pax3 and Otp expressing cells in the $\mathrm{r} 1$ mantle; these do not extend into the Is mantle (Allen Developing Mouse Brain Atlas, http://developing mouse.brain-map.org/). Additional isthmic landmarks are provided by the ventricular isthmic fossa at the midline floor area, plus the paramedian trochlear motor nucleus, which jointly allowed us to delimit the Is tegmentum from that of the ventral midbrain (Palmgren 1921; Vaage 1969, 1973; Kuhlenbeck 1973). Moreover, the decussation of the superior cerebellar peduncle (xscp) was estimated to lie just rostral to the Is/r 1 boundary as defined by the mentioned gene markers. The isthmic serotonergic populations are represented mainly by what we call the isthmic dorsal raphe nucleus (isDR), which is the largest part of the conventional dorsal raphe nucleus (commonly wrongly 

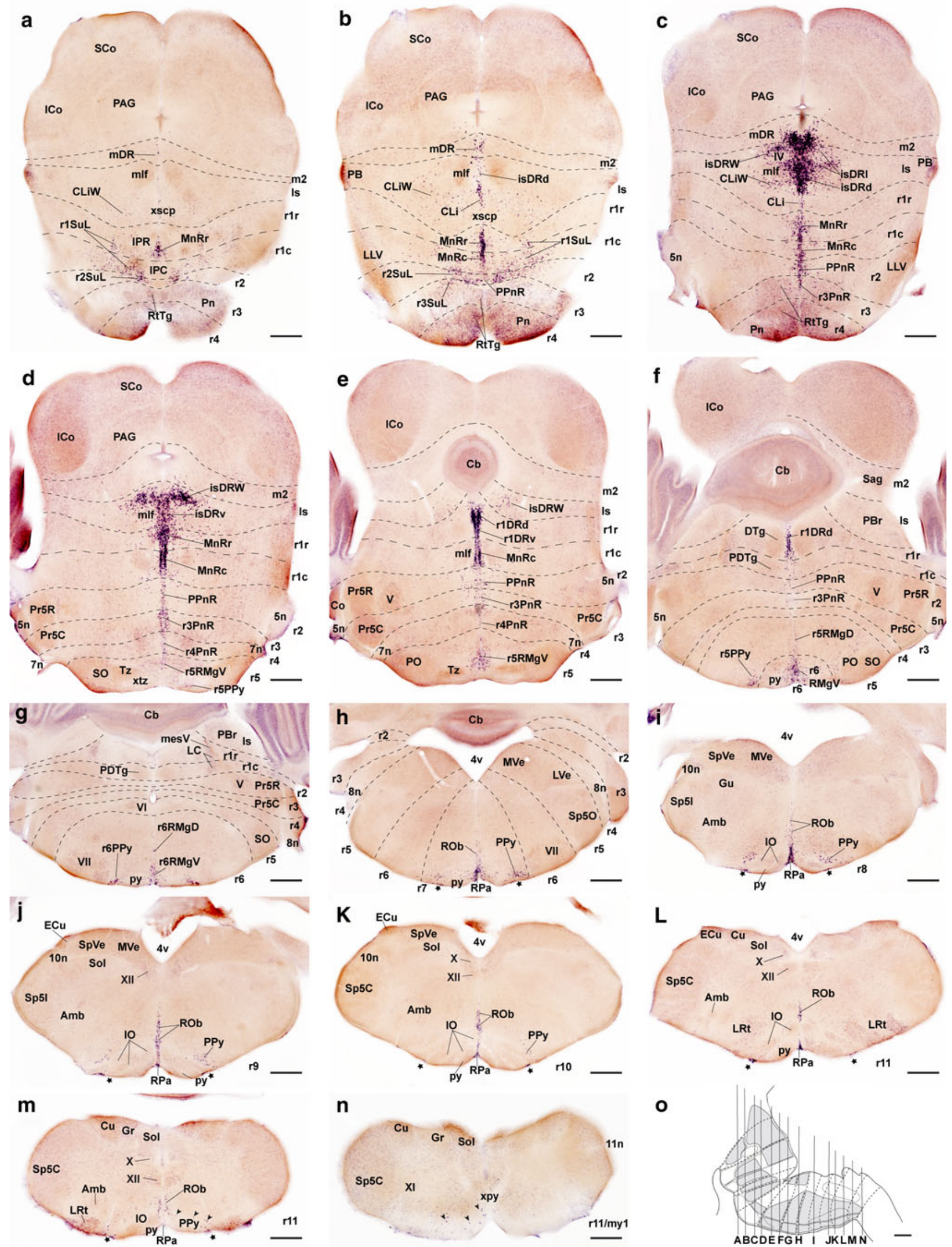

o

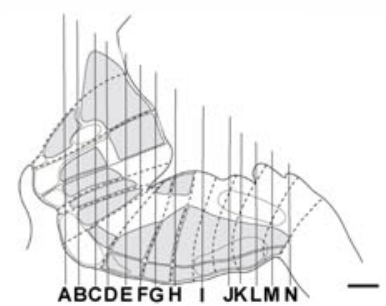


held to lie in the midbrain; compare Fig. 1); this is composed of dorsal, ventral, lateral and wing subdivisions (isDRd, isDRv, isDRl, isDRW). The isDR is complemented by a much sparser serotonergic population found at the caudal linear nucleus (CLi), including laterally dispersed cells, or wing portion of the CLi (CLiW).

The isDR is easily recognizable in paramedian sagittal sections, because it shows a considerable density of its 5-HT-positive cells, contrasting with its caudal neighbor, the less populated rl dorsal raphe nucleus (r1DR) (Figs. 1a-c, 2b-f, 3a). The isDRd occupies the ventral midline of the isthmic periaqueductal stratum, while the isDRv lies ventral to the former at the corresponding deep intermediate stratum, just medial to the trochlear motor nucleus; the isDRl caps bilaterally the trochlear nucleus and the medial longitudinal fascicle (IV, mlf; Figs. 1, 2b-d, 3a-d). Similar neurons appear dispersed even more laterally, in what may be classified as wings of the isDR (isDRW); these cells lie either inside or outside the periaqueductal gray (ventrolateral DR of Paxinos and Franklin 2007). Comparison of isDR with the isthmomesencephalic boundary (passing in sagittal sections between the oculomotor and trochlear nuclei; Palmgren 1921; Vaage 1973; Joyner et al. 2000; Zervas et al. 2004; Puelles et al. 2012b) strongly suggests that a rostral subgroup of 5-HT-positive cells of the DR complex actually lies within the caudal midbrain (Figs. 1a, 3a, b). Puelles et al. (2012b) have recently proposed that this midbrain DR formation lies specifically at the periaqueductal midline within the mesomere 2 , that is, at the caudalmost, preisthmic developmental subregion of the midbrain, a locus also rich in dopaminergic neurons. We will examine below the issue whether these cells arise in the midbrain, or result from a migration originated in the isthmus, as was already suggested by Zervas et al. (2004). In any case, we named this rostral pole of the DR complex the midbrain dorsal raphe nucleus (mDR), according to its adult topography (note the literature often takes the entire DR complex as being mesencephalic; see "Discussion").

The CLi is populated by dispersed serotonin-immunoreactive neurons distributed at intermediate radial levels of the isthmic paramedian tegmentum (they do not extend into the superficially placed isthmic part of the interpeduncular nucleus; Fig. 1). This paramedian isthmic tegmental area is intercalated rostrocaudally between the dorsal and ventral tegmental decussations of the midbrain and the decussation of the superior cerebellar peduncle found within caudal Is. The CLi cells are less abundant and compact than those of the isDR, and do not invade the midbrain (Figs. 1a, 2b, c, 3a-e). Laterally to the CLi nucleus, a serotonergic winglike population appears, with similar cell density than the CLi. We named this serotonergic group the caudal linear wing nucleus (CLiW; Fig. 2b, c).
Fig. 3 Pet 1 detected by in situ hybridization in a series of consecutive sagittal sections proceeding from medial to lateral at $\mathrm{P} 0$. The rhombomeric boundaries are traced as white dashed lines (smaller dashes separate the rostral and caudal halves of r1). a, b Paramedian levels; compare a with the P10 distribution shown in Fig. 1a. c-h More lateral levels, showing the migrated wing and ventrolateral raphe formations. For abbreviations see "List of abbreviations". Scale bar $500 \mu \mathrm{m}$

R1 raphe nuclei The periventricular $\mathrm{r} 1$ domain contains what we call the caudal or $\mathrm{r} 1$ portion of the dorsal raphe nucleus (r1DR), as well as the median raphe group (MnR) and a large superficial ventrolateral aggregate ( $1 \mathrm{SuL})$. The MnR (a.k.a. central superior nucleus; Table 1) extends as defined conventionally also into $\mathrm{r} 2$, but for clarity we propose recognizing two distinct nuclei here, reserving the MnR name for $\mathrm{r} 1$ (see below as regards $\mathrm{r}$ ). The supralemniscal $\mathrm{r} 1$ raphe nucleus (r1SuL) corresponds to the larger rostral part of a ventrolaterally displaced cell population that extends likewise into $r 2$ and $r 3$, at least (Table 1). To pinpoint the rostro-caudal limit of $\mathrm{r} 1$ and, therefore, of these nuclei, we used as before the selective widespread tegmental distribution of $\operatorname{Pax} 7$ and Pax3 positive cells in the $\mathrm{r} 1$ mantle layer, as well as the selective expression of Otx2 in a basal paramedian population restricted to the caudal half of $\mathrm{r} 1$ (unlabeled radial patch in Fig. 4b; Lorente-Cánovas et al. 2012; Puelles, unpublished observations; Allen Developing Mouse Brain Atlas, http://developingmouse.brain-map.org/). Remarkably, the Otx2 pattern corroborates molecularly distinct rostral and caudal halves of $\mathrm{r} 1$ (a point previously deduced from differential histogenetic patterns by Vaage 1973; this author noted that $\mathrm{r} 1$ is about double as large as a normal rhombomere); we identify here these parts as $\mathrm{r} 1 \mathrm{r}$ and $\mathrm{rlc}$ (Fig. 1). Moreover, the early expression of Hoxa 2 characteristically ends rostrally at the r1-r2 boundary (Irving and Mason 2000; Moens and Prince 2002; Aroca and Puelles 2005; Oury et al. 2006; Lorente-Cánovas et al. 2012; Puelles, unpublished observations).

The r1DR portion of the dorsal raphe nucleus is on the whole less populated than the isthmic portion, and is found largely in paramedian periventricular position within the rostral and caudal parts of $\mathrm{r} 1$, though predominantly in the rostral part (r1DR; Figs. 1, 2e, f, 3a). In contrast to the isDR, the r1DR shows only a sparse laterally placed wing portion (r1DRW), but dorsal (periaqueductal) and ventral (intermediate) subnuclei can be distinguished, as in the isthmus (r1DRd; r1DRv; Figs. 1, 2e, f, 3a); the r1DRv is the only component that extends into r1c (Fig. 1b). Its periaqueductal or dorsal cell mass is intercalated at the midline between the bilateral dorsal tegmental nuclei, whereas the ventral portion separates similarly the ventral tegmental in r1c nuclei (DTg, VTg; Figs. 1, 2e, f, 3a); the 

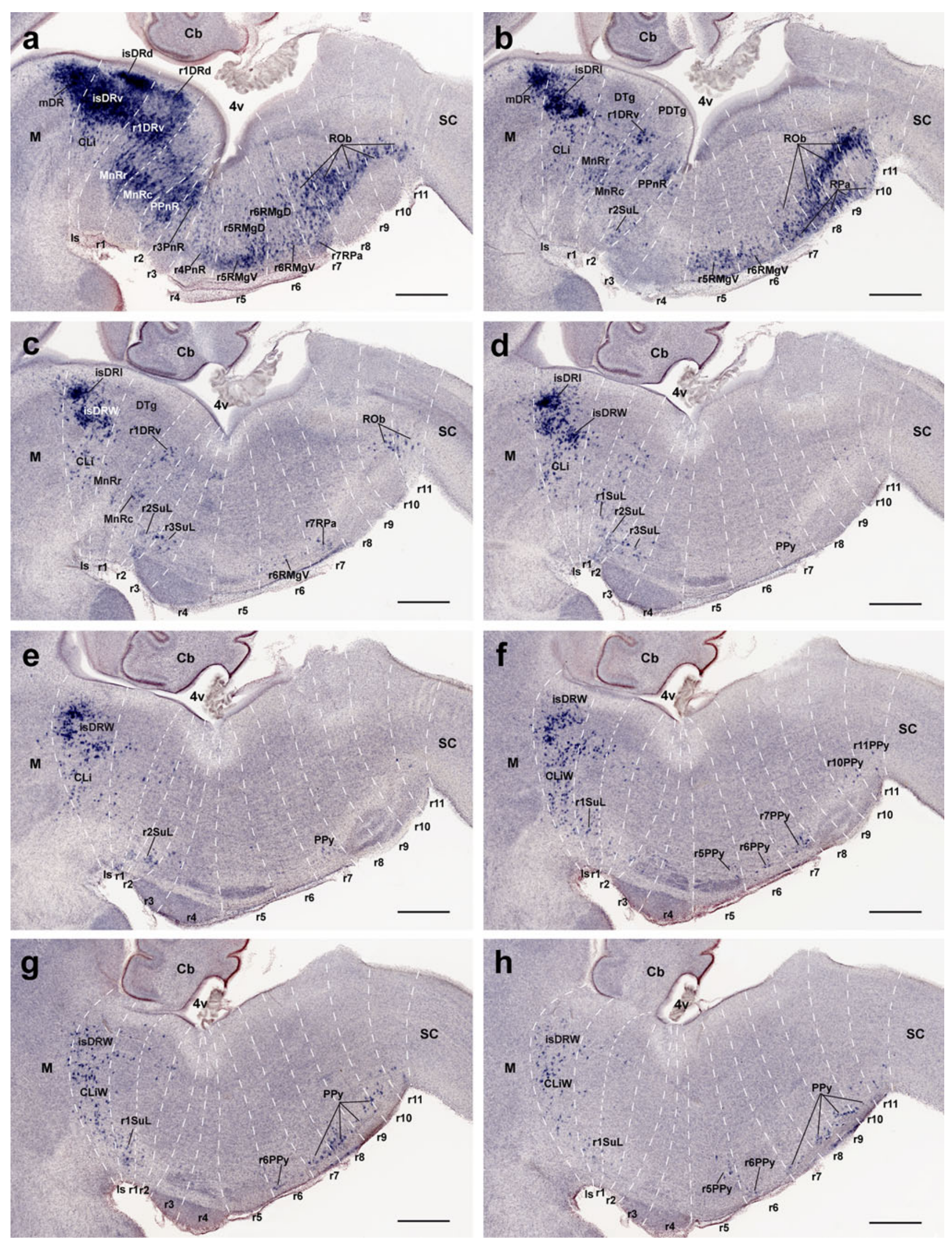
r1DRv relates to the posterodorsal tegmental nucleus (PDTg; Fig. 15). There are very few midline serotonergic cells at the caudal half of $\mathrm{r} 1$, next to the posterodorsal tegmental nucleus (Figs. 1, 3a, 15). It does not seem meaningful to identify these sparse caudal $r 1$ neurons as a straightforward raphe nucleus population, associated or not to the DR.

In contrast, the median raphe nucleus (MnR), presently redefined by us so that the $\mathrm{r} 2$ analog is excluded from this concept, occupies both halves of $r 1$; there is no obvious separation between the $\mathrm{r} 1 \mathrm{r}$ and $\mathrm{r} 1 \mathrm{c}$ moieties at postnatal stages. This cell group lies across the median glial raphe at intermediate levels of the $\mathrm{r} 1$ tegmentum, deep to the $\mathrm{r} 1$ parts of the interpeduncular nucleus (MnR, IPR, IPC; Fig. 1). Its neurons appear sharply separated from the periaqueductal r1DR formation (Figs. 1, 2a-f, 3a-d), though sparser serotonergic cells can be found occasionally in-between. The r1r part of $\mathrm{MnR}(\mathrm{MnRr})$ respects rostrally the paramedian space occupied by the apical interpeduncular and rhabdoid nuclei, and similarly respects superficially (ventrally) the space occupied by the rostral interpeduncular nucleus (IPA, Rbd, IPR; Fig. 1). The MnRr is slightly larger than its r1c counterpart $(\mathrm{MnRc})$, and apparently contains more 5-HTimmunoreactive cells (Figs. 1, 2c-f, 3a). The MnRc lies medial to the Otx2-positive tegmental population in the paramedian basal plate of caudal r1 (data not shown; see Allen Atlas; Lorente-Cánovas et al. 2012), and is separated from the pial surface by the caudal interpeduncular nucleus (IPC; Figs. 1a, 2a, 3a).

Apart of MnR, there is also a sizeable population of $\mathrm{r} 1$ serotonergic cells that appear displaced laterally from the median raphe neighborhood. Such elements are rather dispersed at intermediate radial levels, where they appear typically just outside of the paramedian locus identified classically as 'paramedian raphe nucleus' (itself devoid of serotonergic neurons). Similar lateral cells tend to form a larger aggregate more superficially (ventrally), abutting the rostral part of the decussation of the trigeminal lemniscus (therefore the 'supralemniscal' descriptor applied by some authors; Table 1), lateral to the interpeduncular nucleus. Given that similar lateral cells appear as well in $\mathrm{r} 2$ and $\mathrm{r} 3$, we identify it as the supralemniscal $r l$ raphe nucleus (r1SuL; Figs. 1, 2a-b, 3d). Similar supralemniscal raphe populations are distinguished in $\mathrm{r} 2$ and $\mathrm{r} 3$ (see below).

$R 2$ raphe nuclei The $\mathrm{r} 2$ basal plate is delimited rostrally by the Pax7/Pax3/Otx2-expressing cells of the caudal interpeduncular subnucleus and overlying basal tegmentum within r1c (see above), and caudally by the pontine nuclei, the related pontine decussation (xpn) and the reticulotegmental nucleus in r3-r4 (Puelles et al. 2007; Fig. 1). In postnatal mice, the $\mathrm{r} 2$ floorplate is much compressed rostrocaudally between the IPC (r1c) and the basilar pons in r3-r4; it typically contains the major part of the decussation of the trigeminal lemniscus. We compared the location of these landmarks with the expression pattern of Hoxa2 and Hoxb2 genes from the Allen Developing Mouse Brain Atlas (http://developingmouse.brain-map.org/). The expression domains of Hoxa2 and Hoxb2, respectively, stop rostrally at the $\mathrm{r} 1 / \mathrm{r} 2$ and $\mathrm{r} 2 / \mathrm{r} 3$ boundaries. Hoxa 2 ISH signal abuts rostrally the $O t \times 2$-positive $\mathrm{r} 1$ domain.

We commented above that the caudal portion of the classic MnR nucleus lies across the $\mathrm{r} 2$ raphe. However, it seems convenient to separate this cell population from the MnR proper (in $\mathrm{r} 1$ ), as is suggested by some differential markers (see below). We therefore called the distinct $\mathrm{r} 2$ population of raphe serotonergic cells the prepontine raphe nucleus (PPnR), on account of its topography relative to the pontine rhombomeres $r 3-r 4$. The major part of PPnR lies across the midline raphe at intermediate radial levels of $\mathrm{r} 2$, starting ventrally just dorsal to the trigeminal lemniscal decussation (PPnR; Figs. 1, 2b-d, 3a-c).

In addition, there are also ventrolaterally displaced superficial serotonergic cells within $\mathrm{r} 2$, which we have named the supralemniscal $r 2$ raphe nucleus (r2SuL; Fig. 2); this cell group is smaller than r1SuL. These neurons are aligned longitudinally with the $\mathrm{r} 1$ and $\mathrm{r} 3$ counterparts (Figs. 2a-b, 3d-e).

$R 3$ and $r 4$ raphe nuclei For these neuromeres we used as medial landmarks the pontine and reticulotegmental nuclei (Pn, RtTg), which invade these two developmental units from E15.5 onwards, after culminating their respective tangential migrations from the rhombic lip. The trigeminal sensory and motor nerve roots emerge laterally at the caudal end of $\mathrm{r} 2$, aiding the delimitation from $\mathrm{r} 3$ (Allen Developing Mouse Brain Atlas, http://developingmouse. brain-map.org/) and the rostral end of the Hoxb2 expression domain likewise delimits $\mathrm{r} 2$ from $\mathrm{r} 3$ (Egr2 - known before as Krox20-labels selectively $\mathrm{r} 3$ and r5, being likewise useful for the present purpose). Moreover, the roots of the facial and vestibulocochlear nerves traverse laterally $\mathrm{r} 4$ (contained wholly within its rostral and caudal limits), and the intraneural course of the facial motor fibers within $\mathrm{r} 4$ (rostral to the genu) also allows a rough estimation of the r3-r4 and r4-r5 boundaries. Both r3 and r4 are considerably compressed rostrocaudally near the fourth ventricle, but expand anteroposteriorly at the subpial pontine basilar complex (Fig. 1).

We found that the $\mathrm{r} 3$ raphe sector clearly contains more serotonergic cells than $\mathrm{r} 4$, though possibly less than $\mathrm{r} 2$ (Figs. 1, 2c-e, 3a). The $\mathrm{r} 3$ paramedian elements represent a distinct, radially elongated pontine $r 3$ raphe nucleus (r3PnR) that extends through intermediate radial levels, immediately caudal to the PPnR in r2 (r3PnR; Figs. 1, 2ce, 3a). More superficial, laterally displaced serotonergic neurons constitute separately the $\mathrm{r} 3 \mathrm{SuL}$ cell group, which 
appears dorsal to the $\mathrm{r} 3$ basilar pons, partly associated to the reticulotegmental nucleus (r3SuL; Figs. 2b, 3c-d); note that at embryonic stages these cells lie subpially (Fig. 5a, $\mathrm{b}, \mathrm{d}, \mathrm{e})$, but apparently result covered by the pontine and reticulotegmental migrations. We found no counterpart of r3SuL within r4.

Serotonin cells are much reduced in number in $\mathrm{r} 4$, but some cells are nevertheless clearly present. Most of these cells are characterized postnatally by low 5-HT-immunoreaction (or low Petl signal), but we also observed a few highly 5-HT-immunoreactive (and Petl expressing) scattered neurons (Fig. 1a). We identified the small paramedian serotonergic aggregate lying at a ventral intermediate radial level just dorsal to the pontine nuclei as the pontine $r 4$ raphe nucleus (r4PnR; Figs. 1, 2d, e, 3a). This validates the expression 'pontine raphe nuclei', restricted to the paramedian raphe components of r3 and r4. Note, however, that this expression has been wrongly used in the literature, applying it to raphe elements lying more rostrally (even up to the Is), due to the misconception that the entire rostral hindbrain is "pontine' (Table 1 and "Discussion").

\section{The caudal cluster}

Traditionally the caudal cluster is held to be formed by the raphe magnus (RMg), the raphe obscurus ( $\mathrm{ROb})$ and the raphe pallidus (RPa) nuclei (e.g., Taber et al., 1960), plus a set of lateralized superficial parapyramidal neurons. These formations represent plurisegmental complexes, once their respective topography relative to the r5-r11 developmental units is taken into account. The most rostral of these populations, the $\mathrm{RMg}$, appears to be distributed across rhombomeres r5 and r6 (these are the 'retropontine' rhombomeres according to Puelles et al. 2007, or the 'pontomedullary' rhombomeric units of the Allen Developing Mouse Brain Atlas), whereas the more caudal ROb and RPa complexes are both distributed rostrocaudally all along the caudal medulla oblongata, which is held to be subdivided into five cryptorhombomeres (r7 to r11) (Cambronero and Puelles 2000; Puelles et al. 2007; Marín et al. 2008). The parapyramidal neurons extend throughout the r5-r11 continuum.

$R 5$ and r6 raphe nuclei The superficial trapezoid body decussation (xtz) and associated auditory grisea (particularly the nucleus of the trapezoid body), which are restricted to $\mathrm{r} 5$, jointly with the facial motor nucleus, that characteristically is located in r6 (after its early migration), allowed us to pinpoint the r5/r6 boundary (Figs. 1, 2d-e; Wolfer et al. 1994; Puelles et al. 2007; Marín et al. 2008). In addition, this limit coincides with the rostral end of Hoxd3 expression (Tümpel et al. 2009; Allen Developing Mouse Brain Atlas (http://developingmouse.brain-map.org/
), whereas the r6/r7 boundary coincides with the rostralmost Hoxd4 expression, and the caudal pole of the migrated facial motor nucleus. The facial efferents also serve as landmarks in this area; they first approach the periventricular genu across the r6 tegmentum; in the genu, the fibers ascend longitudinally through r5 (bypassing the abducens motor nucleus), and then course transversally into the nerve root within $\mathrm{r} 4$ (Figs. 1, $2 \mathrm{~d}$, e).

At P10 we observed small-sized periventricular serotonergic cells in both $\mathrm{r} 5$ and r6, associated topographically to the facial genu, which we identified as supragenual raphe cell groups (r5SGeR and r6SGeR; Paxinos and Watson 2007) (Fig. 1a, b). There are also typical paramedian raphe cells at r5 and r6, as well as other serotonergic elements that are displaced laterally and superficially into a ventrolateral subpial area. We assigned the paramedian cells to the RMg complex, largely on the basis of shared gene expression patterns (see below). We distinguished therein dense ventral populations present at the superficial stratum-r5RMgV, r6RMgV-from rather dispersed dorsal populations found within the intermediate stratumr5RMgD, r6RMgD (Figs. 1, 2d-g, 3a-c). Moreover, we identified the ventrolateral subpial cell groups as the parapyramidal raphe formation of the corresponding rhombomeres (r5PPy and r6PPy) (Figs. 1, 2f-h, 3f-h). See Table 1 for other terminologies.

R7 to rll raphe nuclei We tentatively located the rhombo-spinal boundary at a plane just caudal to the decussation of the pyramidal tract (xpy). The inferior olive complex extends between r8 and r11, appearing divided into two blocks corresponding to r8-r9 and r10-r11. The raphe obscurus (ROb) and raphe pallidus ( $\mathrm{RPa}$ ) nuclei extend rather uniformly along the paramedian basal plate of the caudal medulla from r7 to r11. RPa lies superficially, ventral to $\mathrm{ROb}$ that is restricted to the intermediate stratum. The ROb ends ventrally just dorsal to the inferior olive complex (IO), while the RPa is found more superficially, just dorsal to the pyramidal tract (py), along the midline that separates the right and left inferior olivary complexes (Figs. 1, 2h-m, 3a-c). Similar cells extend laterally from the RPa, encapsulating the pyramidal tract down to the pia lateral to it. These elements may be identified collectively as the medullary parapyramidal nucleus, with the corresponding segmental portions across r7-r11 (PPy) (Figs. 1, $2 \mathrm{~h}-\mathrm{m}, 3 \mathrm{f}-\mathrm{h})$. We also found some scattered median serotonin-immunoreactive cells caudal to the pyramidal decussation (xpy), that is, in the rostralmost spinal cord (Figs. 1, 2n). These may represent RPa cells dispersed caudalwards; they were more abundant at early developmental stages (see below). The most caudal neurons of the $\mathrm{ROb}$ are embedded in the pyramidal decussation (Fig. 2n). 


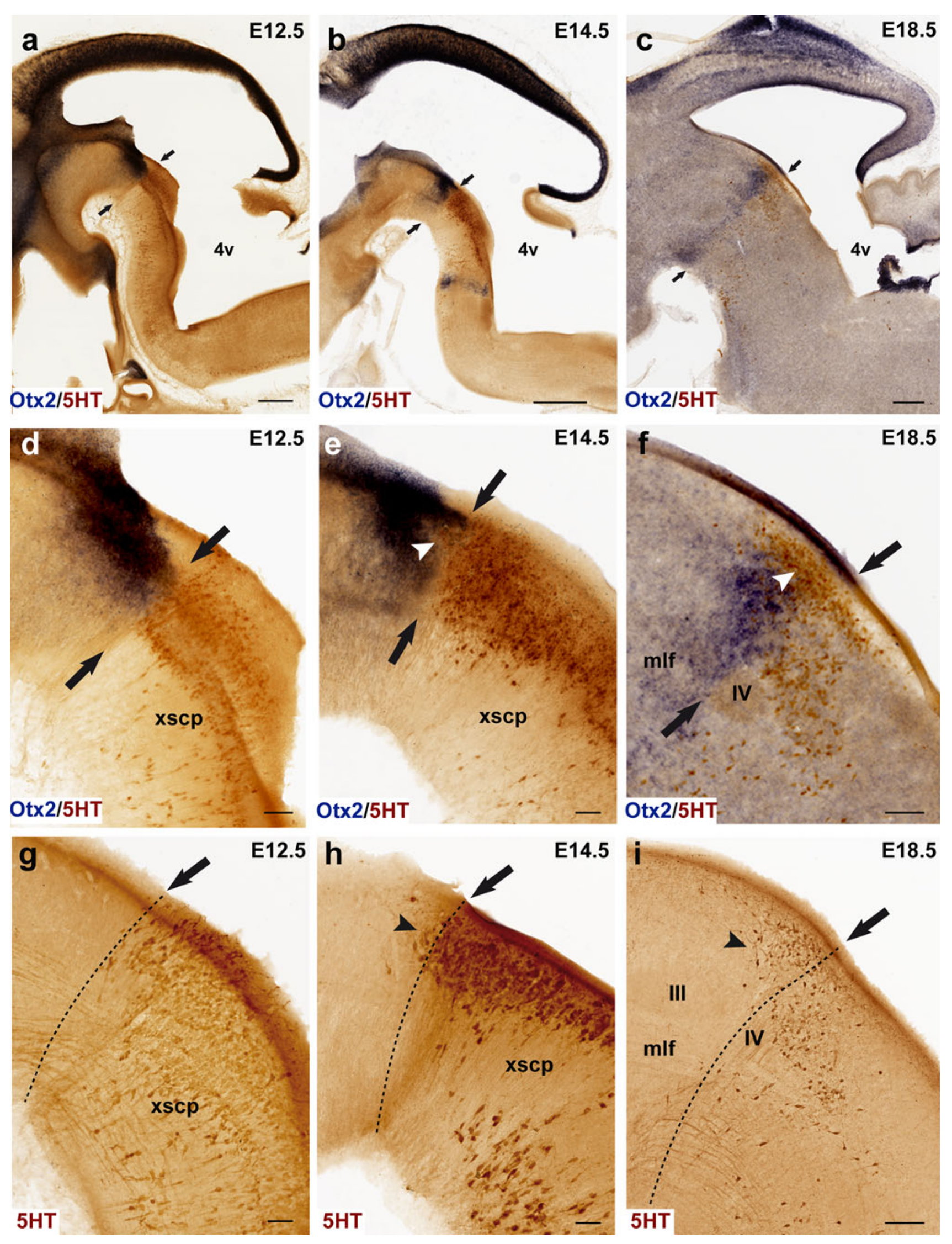


4Fig. 4 Late appearance of the mesencephalic part of the dorsal raphe nucleus. The issue whether any raphe neurons arise in the midbrain is approached here by comparing 5-HT-immunoreaction with Otx2 in situ hybridization on the same sections, at different stages (Otx2 is known to mark sharply the caudal boundary of the midbrain). a-c Low-magnification images of 5-HT (brown) and Otx2 (blue) signals at the hindbrain-midbrain boundary in paramedian sagittal sections at three different developmental stages. Arrows indicate the midbrain/ hindbrain boundary, as marked by Otx 2 expression (consistently with parallel fate-mapping data). $\mathbf{d}-\mathbf{f}$ Higher magnification of the sections in a-c, respectively, showing details of the changing topographic relationship of serotonergic neurons with the midbrain boundary. No midbrain 5-HT cells are observed at E12.5 (d), but a few become apparent at E14.5 (e); these occupy a small triangular area (marked by white arrowhead) in front of the boundary (arrows), where Otx2 expression seems to be partially downregulated. At E18.5 (f) a discrete group of 5-HT-positive cells appears in front of the boundary (arrows; note also the isthmic landmark provided by the trochlear nucleus, $I V$ ); these serotonergic elements are located mostly in a periventricular stratum that is Otx2-negative (white arrowhead), though the ventrally adjacent $O t \times 2$-positive periaqueductal gray also shows some dispersed serotonergic neurons. This cell group is interpreted by us as $\mathrm{mDR}$, and lies only in the caudal preisthmic midbrain (mesomere 2; compare c). g-i Sagittal sections equivalent to those shown in $\mathbf{d}-\mathbf{f}$ (same stages, respectively), but illustrate only 5-HT immunoreacted cells (corresponding low-magnification images are shown in Fig. 5a-c). The midbrain-hindbrain boundary is marked by a dashed line, and black arrowheads indicate the emergent $\mathrm{mDR}$ neurons. $4 v$ fourth ventricle, III oculomotor nucleus, IV trochlear nucleus, $m l f$ medial longitudinal fasciculus, $x s c p$ decussation of the superior cerebellar peduncle. Scale bar $200 \mu \mathrm{m}$ in a, $500 \mu \mathrm{m}$ in b, $400 \mu \mathrm{m}$ in $\mathbf{c}, 50 \mu \mathrm{m}$ in $\mathbf{d}-\mathbf{e}$ and $\mathbf{g}-\mathbf{h}, 100 \mu \mathrm{m}$ and in $\mathbf{f}$ and $\mathbf{i}$

Formation of raphe nuclei Rostral cluster (Fig. 5): In the mouse, most serotonergic raphe neurons are born by E12.5. At that stage the rostral cluster is largely represented by three plurineuromeric pronuclei. One is the anlage of the $\mathrm{DR}$, and consists of cells aggregated periventricularly next to the midline. This periventricular pronucleus starts immediately behind the midbrain-hindbrain boundary (MHB), where it is most dense, and becomes sparser caudalwards, stopping roughly at the boundary between rostral and caudal parts of r1 (Fig. 5a). It extends therefore across isthmus and rostral $\mathrm{r} 1$, clearly containing the primordia of the isDR and r1DR (note the mDR cells only start to be visible at the rostral end of this column at E14.5; Figs. 4, 5a, b). These nuclei are well formed by E18.5 (Fig. 5c). Only occasional serotonergic cells are found periventricularly at the levels of caudal $\mathrm{r} 1$ and $\mathrm{r} 2$, though a larger population appears in $\mathrm{r} 3$ (see below). Secondly, a separate paramedian population of serotonergic neurons is found more superficially at E12.5, adjacent to the median glial raphe, and partially approaching the pial surface, due to the momentary lack of the interpeduncular nucleus (Fig. 5a). This pronucleus starts at rostral $\mathrm{r} 1$ levels, and extends through caudal $\mathrm{r} 1, \mathrm{r} 2$ and $\mathrm{r} 3$ levels, representing a common primordium for the MnR, PPnR and r3PnR nuclei. A much sparser similar migrated population was observed within r4 (r4PnR; Fig. 5a, g). No such paramedian aggregate appears next to the midbrain (at the Is), though locally dispersed elements form a primordium of the CLi (Fig. 5a). At several levels across r1-r3, isolated cells appear at midcourse between the periventricular stratum and the MnR-PPnR-r3PnR pronucleus in the intermediate zone. Some serotonin neurons persist at such intermediate positions at later stages (E14.5, E18.5; Fig. 5b, c). It was characteristic of $\mathrm{r} 2$ that the PPnR elements extend continuously across the periventricular and intermediate strata. Finally, at E12.5 a separate ventrolateral pronucleus contains the future supralemniscal cells that migrate into positions close to the ventrolateral surface, apparently following a lateral radial course parallel to that of the larger paramedian migrated mass. The paramedian and ventrolateral migrated pronuclei are roughly coextensive rostrocaudally, but the ventrolateral cells are most abundant within $\mathrm{r} 1$, sharply decreasing in number more caudally. These three pronuclear groups do not change essentially at E14.5 and E18.5, irrespective of the sharpening of the individual components (Fig. 5).

Caudal cluster (Fig. 6): At E12.5, many serotonergic cells are already present ventrally next to the median glial raphe throughout the r5-r11 continuum, probably forming the primordia at least of the $\mathrm{RMg}$ and $\mathrm{RPa}$. The $\mathrm{ROb}$ population may be added subsequently. Remarkably, there appears practically no periventricular differentiation of serotonergic cells in the pontomedullary and medullary domains. The small supragenual serotonergic cells only were observed postnatally, at P10 (Fig. 1). At the brain surface, the ventrolaterally displaced PPy pronucleus is established as well by E12.5 (Fig. 6b). These groups do not change essentially at E14.5 and E18.5 (Fig. 6).

Midbrain serotonergic cells We corroborated the existence at P10 of a midbrain dorsal raphe cell group (mDR), which recently was attributed to the midbrain mesomere 2 (m2) by Puelles et al. (2012b). As opposed to the conventional idea that the whole DR complex lies in the midbrain, we thus confirm that only this small component lies rostral to the MHB, having now tested this point with the specific Otx2 midbrain marker. The small $\mathrm{m} 2$ developmental unit was originally postulated by Palmgren (1921) and Vaage (1969, 1973), and resurfaced again in recent years due to molecular and experimental evidence shown in the chick by Hidalgo-Sánchez et al. (2005)—who called it 'preisthmus' - and in the mouse by Puelles et al. (2012b); see also m2 in Martínez et al. (2012), Puelles et al. (2012a) and the abundant mouse genoarchitectonic evidence available in the Allen Developing Mouse Brain Atlas (http://www.developingmouse.brain-map.org).

Leaving apart a group of Pet1-positive cells observed at the same locus at E12.5 (Fig. 7f), serotonergic 5HTimmunoreactive neurons start to appear in the preisthmic 


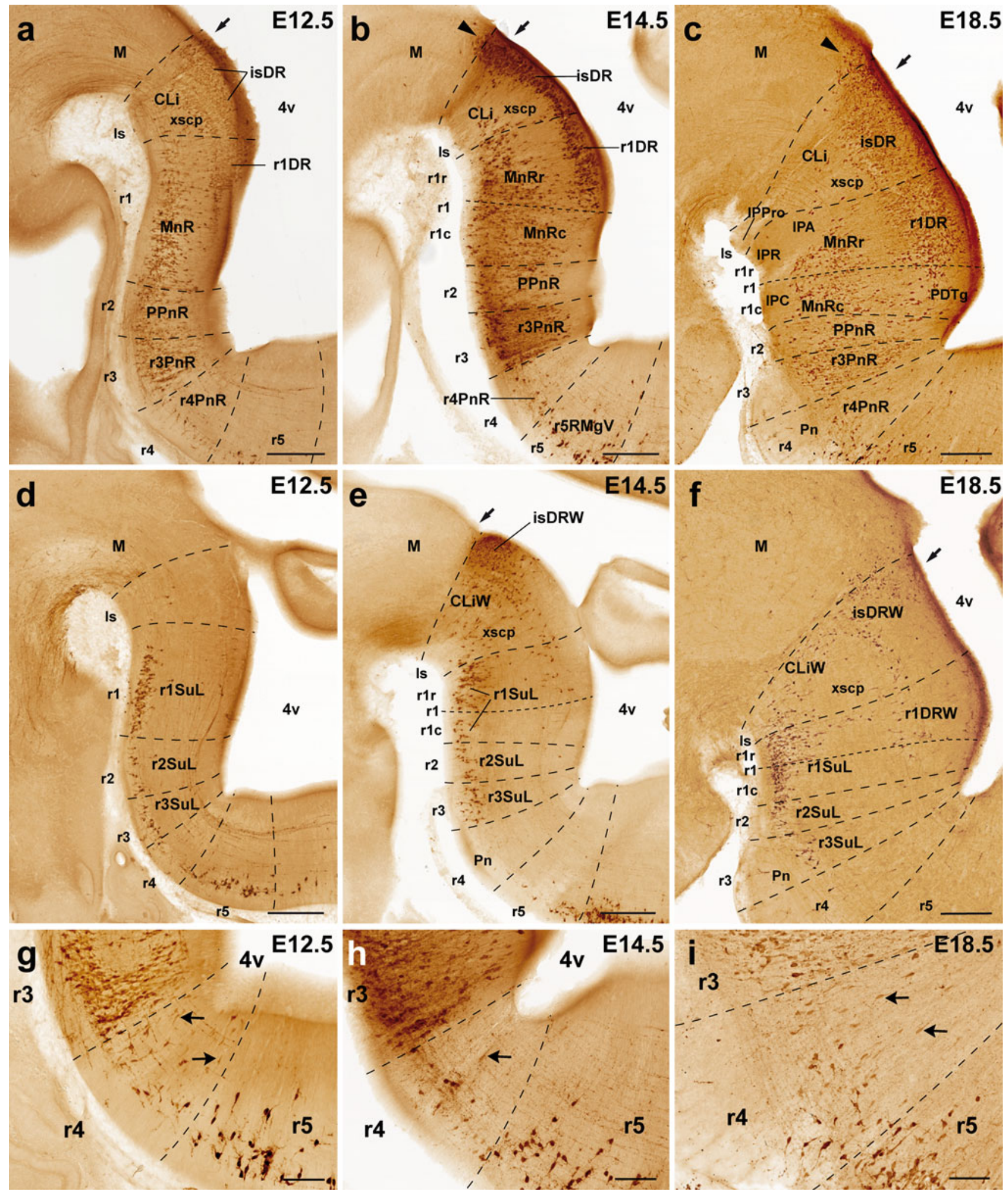

midbrain at E14.5, shaped as a rostrally pointing spike connected with the isDR across the molecular $\mathrm{M} / \mathrm{R}$ boundary delineated by Otx2 (Fig. 4a, b, d, e, g, h). Their number increases significantly by E18.5 (Fig. 4c, f, i).
Curiously, expression of Otx2 seems reduced or even absent at the place occupied by these cells. The genetic profile at E14.5 of the mDR resembles that of its caudal neighbor, the isDR, which suggests that these neurons may 
4 Fig. 5 Segmental mapping of the rostral raphe cluster during embryonic development. 5-HT immunoreactive neurons observed in sagittal sections of mouse brains at E12.5 (a, d), E14.5 (b, e), and E18.5 (c, f), with superposed tracing of postulated interrhombomeric boundaries (dashed lines), and our tentative identification of the nuclear primordia (Table 1). Each set of three images read from left to right (e.g., a-c) represents a temporal sequence at a given section plane. Arrows mark the midbrain-hindbrain boundary. a-f Rostral cluster at paramedian section level. d-f Rostral cluster at a more lateral level. g-i Details at higher magnification of the paramedian pontine region of Fig. 5a-c, respectively. Note some 5-HT immunopositive cells are always present in $\mathrm{r} 4$, mainly in its superficial stratum, though cells with weaker immunoreaction are also observed in the intermediate stratum (arrows in $\mathbf{g - \mathbf { i }}$ ). For abbreviations see "List of abbreviations". Scale bar $250 \mu \mathrm{m}$ in $\mathbf{a}-\mathbf{f}, 100 \mu \mathrm{m}$ in $\mathbf{g - i}$

migrate tangentially from the isthmus, where they would be born.

Genoarchitectonic labeling of raphe nuclei A number of genes have been related to the differentiation of the serotonergic neurotransmitter phenotype. We chose eight of these markers and compared their expression patterns relative to our tentative map of rhombomeric units and the observed raphe nuclei primordia at E12.5, E14.5, E16.5 and E18.5 (E16.5 data not shown). We were interested in gene expression patterns indicative of either neuromeric heterogeneity (unique regional pattern) or metamery (repeated pattern). We also assessed molecular peculiarities accompanying the variable radial stratification of the diverse serotonergic populations. We mapped the transcription factors En1, En2, Gata2, Gata3, Lmx1b, and Pet1, known to act upstream of the serotonergic phenotype (among other roles), as well as Tph2 (coding the ratelimiting enzyme that synthesizes serotonin) and Slc6a4 (the solute carrier family 6 member 4, also known as the serotonin transporter). In addition, we examined and downloaded other data available in public databases (e.g., the Allen Developmental Mouse Brain Atlas). Our results revealed a common differentiation program of hindbrain serotonergic cells, with subtle neuromeric differences in the expression pattern of these genes and the stratification of the derived raphe populations, suggesting that a specific combination of signals applies to each rhombomeric raphe domain (summarized in Fig. 13).

For simplicity, we will present these data grouped into gene pairs mutually compared through the three developmental stages studied, dividing the material in two blocks dedicated to the rostral and caudal raphe clusters, respectively.

\section{The rostral raphe cluster}

En1 and En2 Both En genes were shown to play a role in the specification of some 5-HT-positive neurons of the rostral cluster (Simon et al. 2005; Wylie et al. 2010; Fox and Deneris 2012). Leaving apart their expression within the midbrain, the genes En1 and En2 are expressed in the rostral hindbrain in a decreasing gradient caudalwards from the isthmic organizer. Enl and En2 differ in spatial range of expression (En1 more extensive than En2, particularly at E12.5; Fig. 7a, b; compare Fig. 9a, b) and in radial distribution of their expression. En2 is largely restricted to ventricular cells at E12.5 and E14.5-absent at E18.5while Enl also appears in postmitotic neurons (Figs. 7a, b, 8a, b, 9a, b). At E12.5 and E14.5, En1 is expressed by the periventricular DR pronucleus in a gradient across Is and rostral r1 (Figs. 7a, i, 8a, i). The derived isDR and r1DR nuclei are also positive at E18.5, the r1DR signal being weaker (Fig. 9a). As regards the intermediate paramedian and ventrolateral pronuclei, again only the CLi and $\mathrm{MnRr}$ paramedian cells (Is, r1r) showed strong Enl signal at E12.5 and E14.5 (Figs. 6a, 7a). The MnRc (r1c) shows faint Enl signal at E14.5; this labeling becomes more distinct at E18.5 (Fig. 9a). At the latter stage, the labeled CLi group appears more populated and is better separated from the DR complex (Fig. 9a). The DR wing portions and the r1SuL also show Enl signal (Fig. 9i), but not so the r2 and r3 SuL analogs. Sparse En1-positive neurons were observed within the periventricular area of caudal $\mathrm{r} 1$, and a very low signal was detected at the PPnR in r2 (Fig. 9a). More caudally in the hindbrain other En1positive neurons are observed, but they are not serotonergic (i.e., not immunoreactive for serotonin). At E18.5, ventricular En2 expression largely has disappeared in medial sagittal sections, but persists at the isthmus more laterally, possibly indicating heterochronic regulation (Fig. 9b, j).

Gata2 and Gata3 Gata2 and Gata3 are expressed in largely overlapping domains in the hindbrain. Gata2 activates genes required for the specification of all 5-HT neurons (upstream of Lmxlb and Petl), while Gata3 function seems necessary only for the development of the caudal cell groups of the raphe (see "Discussion"). At E12.5, expression of both Gata2 and Gata3 is intense in the periventricular raphe pronucleus of the rostral cluster (but the positive column remarkably extends as far back as r6) (Fig. 7c, d, k, 1). Gata2 shows much weaker signal than Gata3 at the incipient intermediate paramedian and ventrolateral pronuclei (Fig. 7c, d, k, 1, q, r). At E14.5, Gata2 expression diminishes to rather weak levels in the major part of the rostral cluster, and disappears completely within r4, though isolated cell groups are still positive at the immature DR and MnR nuclei (Fig. 8c, k, q). Gata3 instead retains its expression at the periventricular and intermediate raphe pronuclei of the rostral cluster (the continuous positive periventricular stratum now stops at the r3/r4 limit; Fig. 81); the ventrolateral superficial column 

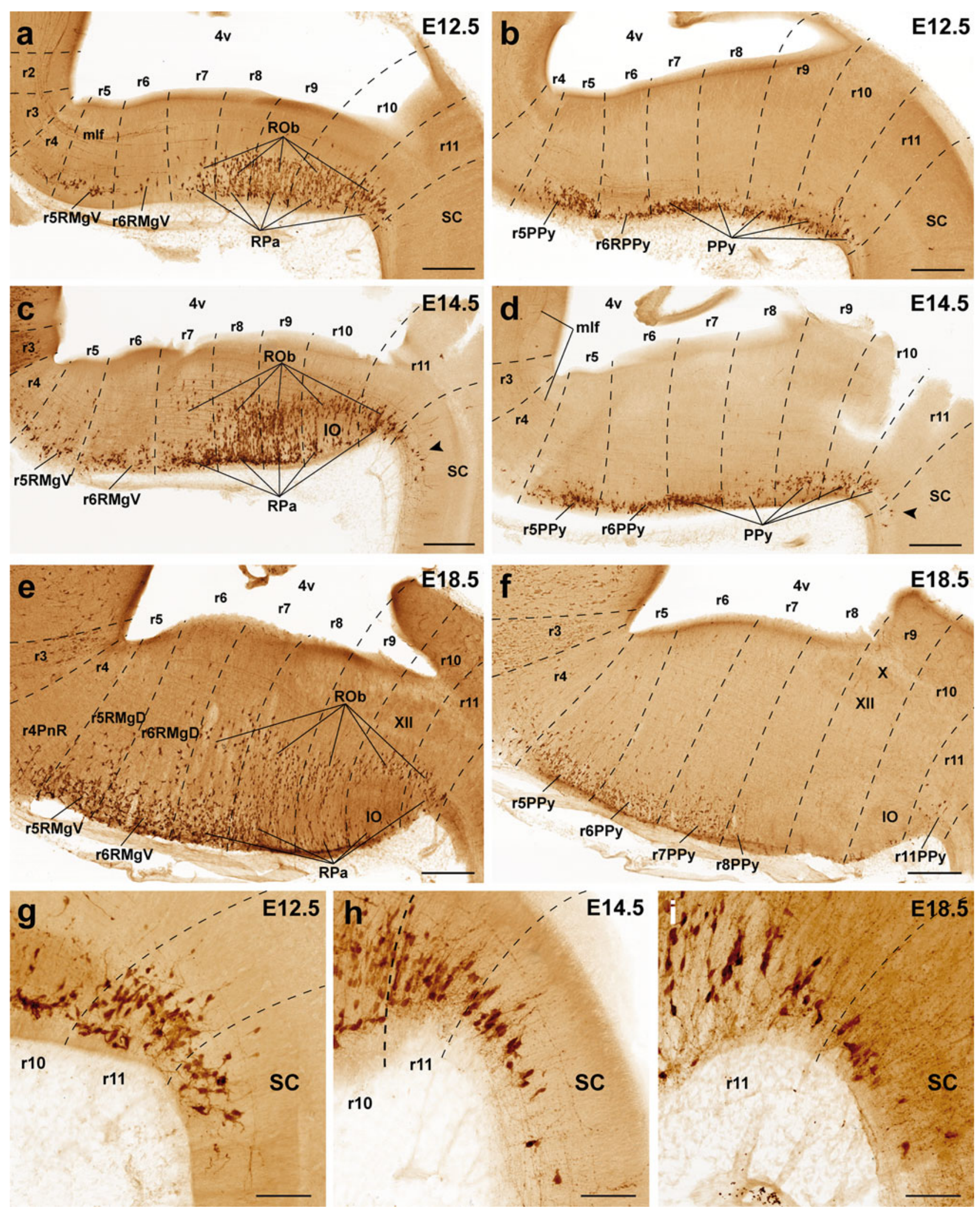

(prospective SuL) does not show significant signal. Maximal Gata3 signal appears at the DR and MnR formations (Fig. 8c, d, k, 1). These differential patterns are even more evident at E18.5 (Fig. 9c, d, k, 1). The periventricular positive cells found in $\mathrm{r} 2$ and $\mathrm{r} 3$ are not immunoreactive for serotonin (Fig. 9d). 
4Fig. 6 Segmental mapping of the caudal raphe cluster during embryonic development. 5-HT immunoreactive neurons observed in sagittal sections of mouse brains at E12.5 (a, b), E14.5 (c, d), and E18.5 (e, f), with superposed tracing of postulated interrhombomeric boundaries (dashed lines), and our tentative identification of the nuclear primordia (Table 1). Each set of three images read from top to bottom (e.g., a, c, e) represents a temporal sequence at a given section plane. a, c, e Findings at paramedian section level. b, d, f Findings at a more lateral sagittal section level. g-i Higher magnification of the rhombo-spinal boundary, showing presence of some 5-HT positive cells in the upper cervical spinal cord. Note that these cells are already present at E12.5 (see also arrowheads in $\mathbf{c}$ and $\mathbf{d}$ ). For abbreviations see "List of abbreviations". Scale bar $300 \mu \mathrm{m}$ in $\mathbf{a}-\mathbf{e}$, and $100 \mu \mathrm{m}$ in $\mathbf{g - \mathbf { i }}$

Lmxlb and Petl Lmxlb and Petl are considered transcription factors linked to the differentiation of postmitotic 5-HT neurons; Lmxlb signal actually extends also within the basal midbrain and forebrain in association to dopaminergic cell populations (both progenitors and neurons; e.g., Fig. 7e, m), whereas Petl is a specific marker of differentiated serotonergic neurons (Hendricks et al. 1999; Cheng et al. 2003; Ding et al. 2003). At E12.5, periventricular Petl signal is strong in the isDR complex (Fig. 7f, $\mathrm{m})$; the CLi and CLiW are not distinguished yet, and a few labeled cells appear within the neighboring midbrain periaqueductal gray (see arrowhead in Fig. 7f; note no serotonin-immunoreactive cells are found at this locus at this stage; Figs. 4g, 5a). The positive periventricular raphe cells are restricted to a sublayer of the periventricular pronucleus next to the ventricular cells themselves; this sublayer is much thinner at r1 levels and becomes again more populated more caudally, extending back at least to r6 levels (Figs. 7f, 10g). At r3 level a paramedian periventricular group of cells occupies the outer part of the periventricular stratum and appears partly connected with the local intermediate pronucleus (primordia of $\mathrm{r} 3 \mathrm{PnR}$ ). No such transitional cells exist at the isthmus, and few of them characterize the two parts of r1, or r2 (Fig. 7f). The intermediate raphe pronucleus formed medially across the $\mathrm{r} 1-\mathrm{r} 3$ continuum also expresses strongly Pet1. The isDRW and r1SuL cell groups are likewise strongly positive, though there are few labeled ventrolateral cells at $\mathrm{r} 2$ and $\mathrm{r} 3$ levels (Fig. 7n). In the hindbrain, Lmxlb seems to be expressed only in postmitotic neurons. However, it is readily apparent that $L m x 1 b$ labels the majority, if not all, of the periventricular postmitotic cells, thus labeling additional cells, apart those identified by Petl and 5-HT-immunoreaction. This occurs not only across isthmus and r1-r3, but also continues caudalwards down to cryptorhombomere r10 (Figs. 7e, 10e). Other periventricular cell fates are clearly involved. The intermediate raphe pronucleus of the rostral cluster and the laterally displaced isDRW and ventrolateral groups are also positive for $L m x l b$ (Fig. 7e, m), though, remarkably, the rostralmost part of the intermediate column (prospective MnRr) apparently lacks altogether
Lmxlb signal, as suggested by comparison of similar sections with Petl ISH and immunoreaction for 5-HT (Fig. 7e, $\mathrm{f}, \mathrm{m}, \mathrm{n}$ ). Other neuronal populations in the rostral hindbrain tegmental mantle (e.g., trochlear motoneurons and the interpeduncular nucleus) do not express $L m x 1 b$. At E14.5, all periventricular populations caudal to the isthmus and $\mathrm{r} 1 \mathrm{r}$ have lost the $L m x I b$ expression previously observed at E12.5 (Fig. 8e). Finally, the Lmxlb signal at E18.5 is readily comparable to that of Pet1, particularly as regards the DR complex, including its mesencephalic component (arrows in Fig. 9e, f) and the scanty periventricular cells in caudal $\mathrm{r} 1$ and $\mathrm{r} 2$ (Fig. 9e, f). The intermediate paramedian complex also shows massive labeling of MnR, PPnR and r3/r4 parts of PnR by both markers (Fig. 9e, f, t, u; note that our selection of comparable images of the DR complex causes that the other populations are not sectioned identically, coming from different specimens, giving the false impression that the intermediate nuclei are not equally labeled; the details in Fig. 9t, u illustrate this point).

Tph2 and Slc6a4 Tph2 and Slc6a4 are genes expressed in differentiated 5-HT neurons. During development, expression of Tph2 is delayed relative to that of Pet1. This is evident at E12.5; most signal is found at the Is and $\mathrm{r} 1$, at the DR, MnR and r1SuL primordia (Fig. 7g, o); levels caudal to $\mathrm{r} 1$ show little $T p h 2$ expression, both periventricularly and more superficially (Fig. 7g, o; compare 7f, n). Between E14.5 and E18.5, the Tph2 pattern gradually approximates that of Pet1, but does not attain a comparable density (e.g., patchy signal in the periventricular stratum and sparse positive cells superficially; Figs. $8 g$, o and $9 g$, o). On the other hand, expression of Slc6a4 in the same rostral raphe primordia is even more delayed relative to Tph2 and Pet1. At the three stages examined, signal obtained with our Slc6a4 probe was weak or moderate in the whole rostral cluster (Figs. 7h, p, 8h, p, 9h, p). Moreover, Tph2 or Slc6a4 cells were practically nonexistent within r4 (Figs. 7u, v, 8u, v, 9u, v), irrespective of the distinct Pet1-expressing and 5HT-immunoreactive cells mentioned above.

\section{The caudal cluster}

The P10 and adult caudal cluster of the raphe is characterized by including periventricular serotonergic cells within r5 and r6 (SGeR; Fig. 1). We did not detect them with any of the genoarchitectonic markers studied during prenatal development (they differentiate postnatally?). Nevertheless, the early embryonic periventricular stratum of the caudal raphe cluster does express transiently differentiation genes characteristic of the serotonergic lineage. At E12.5, this locus is characterized by either uniform or patchy expression of Gata2, Lmxlb and Pet1 

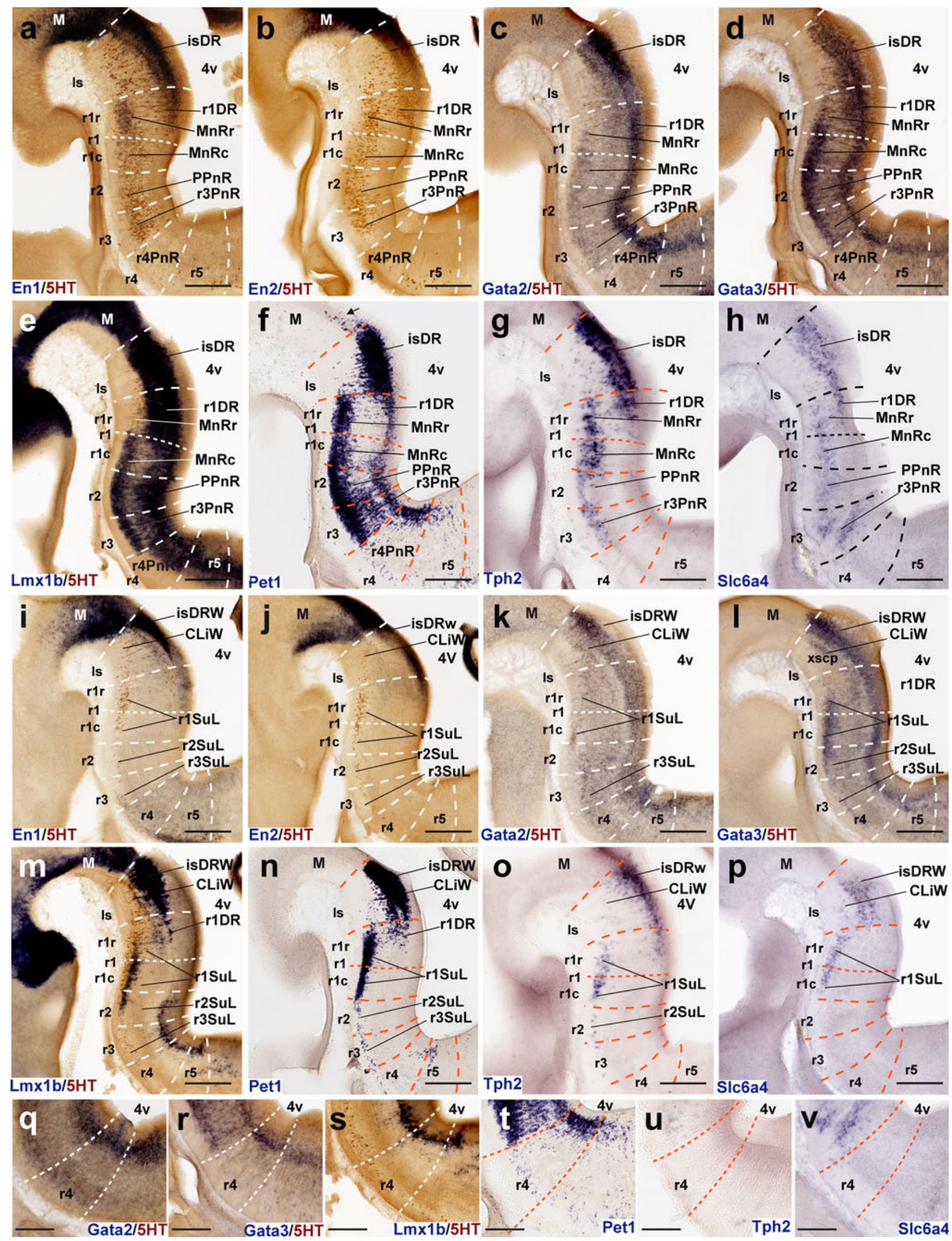

r4 
4Fig. 7 Expression of genes related with the serotonergic phenotype in the rostral raphe cluster in sagittal sections at E12.5. a-h Paramedian sections; the panels show 5-HT immunoreaction plus a particular in situ hybridization (a-e) or only an in situ hybridization (f-h). i-p More lateral section level; i-m show double 5-HT immunoreaction plus in situ hybridization, and $\mathbf{n}-\mathbf{p}$ only in situ hybridization. q-v Higher magnification of $r 4$ in six equivalent paramedian sections with 5-HT immunoreaction plus in situ hybridization (q-s), or only in situ hybridization $(\mathbf{t}-\mathbf{v})$. The riboprobes used are indicated in each case in blue color at the lower left (a-q) or lower right $(\mathbf{r}-\mathbf{v})$ corner. Arrow in $\mathbf{f}$ points to the $\mathrm{mDR}$ nucleus. Scale bar $250 \mu \mathrm{m}$ in $\mathbf{a}-\mathbf{p}$, and $150 \mu \mathrm{m}$ in $\mathbf{q}-\mathbf{v}$

(continuing the patterns noted at the rostral cluster). These expression domains extend caudalwards at least into r10 (Fig. 10a, e, g). Gata3 is expressed similarly, but more weakly (Fig. 10c). These pontomedullary and medullary periventricular populations decrease in importance, or disappear, at subsequent stages, either due to downregulation of the markers or depletion of the stratum via radial migration into the intermediate and superficial strata (Figs. 10a, c, e, g, 11a, c, e, g, 12a, c, e, g). However, some remnants persist at least until E18.5 (e.g., sparse periventricular Lmxlb-positive cells in Fig. 12f); such elements may be the precursors of the eventual supragenual raphe population at r5-r6. We next examine intermediate- and superficial-paramedian and parapyramidal raphe populations, emphasizing differences between the retropontine rhombomeres $\mathrm{r} 5-\mathrm{r} 6$ and cryptorhombomeres r7-r11.

Gata2 and Gata3 At E12.5 Gata2 and Gata3 are generally weakly expressed in the intermediate stratum across the whole caudal cluster (Fig. 10a-d), whereas there is stronger expression at the superficial stratum, particularly in its ventrolateral (parapyramidal) part. The intermediate pronucleus gives rise to the $\mathrm{RMgD}$ in $\mathrm{r} 5-\mathrm{r} 6$ and the $\mathrm{ROb}$ in r7-r11 (Figs. 11a-d, 12a-d). Gata2 signal predominates in the PPy primordium in $\mathrm{r} 5$ and $\mathrm{r} 6$, but is practically absent at the medullary parts of PPy (Fig. 10a, b). Gata3 signal is even stronger at the r5/r6PPy, and extends also into $\mathrm{r} 7$ and r8 parts of PPy (Fig. 10c). None of them labels significantly the RMgV or RPa. At E14.5, Gata2 and Gata3 continue strongly expressed at the r5/r6PPy nucleus (Fig. 11b, d), and start to show signal at the $\mathrm{RMgV}$ (Fig. 11a-b). The medullary PPy primordium has relatively weaker Gata3 signal (Fig. 11d). Weak paramedian superficial signal of the two markers appears in r7-r8, corresponding to rostral parts of the RPa primordium (Fig. 11a, c). At E18.5, expression of Gata2 and Gata3 has decreased significantly, particularly in r9-r11, while more rostrally the $\mathrm{RMgV}$ and PPy (r5-r6) as well as the RPa and medullary PPy populations (r7-r8) partially retain some Gata3 expression (Fig. 12a-d).
Lmxlb and Pet1 At E12.5, Lmxlb is expressed in the r8r10 subdivisions of the ROb/RPa pronuclei (Fig. 10e), while Petl is intense in all groups of this caudal cluster, including cells in r11 (Fig. 10g). Expression of Lmxlb is rather weak and patchy at the $\mathrm{RMgV}$ and PPy, whereas Pet1 appears intensely expressed at the PPy (Fig. 10h) and $\mathrm{RMgV}$ (not shown). At E14.5, a similar differential pattern of Lmxlb and Petl is observed at the ROb/RPa (Fig. 11e, $\mathrm{g})$. The $\mathrm{RMgV}$ and PPy nuclei express moderate levels of Lmxlb (Fig. 11e, f), whereas their Petl signal is strong (Fig. 11g, h). At E18.5, Lmxlb signal, previously already absent at $\mathrm{r} 11$, seems now to have been wholly downregulated at $\mathrm{r} 10$, and is partly reduced at $\mathrm{r} 9$, where we detected many serotonin-positive cells negative for this gene. In contrast, its signal is still apparent at all pontomedullary and medullary raphe groups rostral to r9 (Fig. 12e, f). In comparison, Pet 1 expression is intense at the caudal cluster in general, specially at the superficial stratum of r6, where the $\mathrm{RMgV}$ and PPy appear very strongly labeled (Fig. 12g, h); subtle variations in the cell number and dispersion of the raphe populations correlate with cryptorhombomeric subdivisions (Fig. 12g, h).

Tph2 and Slc6a4 Both genes are weakly expressed at the caudal complex at E12.5, their signal being barely detectable at the ROb/RPa primordia across r8-r10 (Fig. 10i, k) and at the ventrolateral PPy populations across r6-r8 (Fig. 10j, 1). At E14.5 Tph2 expression appears now strongly at the $\mathrm{RMgV}$ and $\mathrm{ROb} / \mathrm{RPa}$ all the way to $\mathrm{r} 11$, but remains weak at the medullary PPy (Fig. 11i, j). In contrast, the Slc6a4 signal remains weak throughout, an aspect that may be due to technical reasons (Fig. $11 \mathrm{k}, 1$ ). At E18.5, intense $T p h 2$ signal is now similarly distributed as Pet1 signal (even extending slightly into the rostral spinal cord; arrow; Fig. 12i, j); the RMgV and PPy raphe groups in $\mathrm{r} 6$ are particularly distinct and other populations show subtle variations coinciding with postulated neuromeric subdivisions (Fig. 12i, j). Slc6a4 expression has increased somewhat, but remains weaker than that of $T p h 2$, and does not extend as far caudally (Fig. 12k, 1).

Genes of the Allen Brain Atlas database with restricted expression pattern in the raphe nuclei

We examined some genes from de Allen Brain Atlas database which showed restricted expression in the postnatal or adult raphe nuclei. We searched specifically for genes which are expressed differentially across the diverse serotonin populations identified in our analysis. It was expected that differences in regionally specific molecular background and/or terminal differentiation might be reflected in patterns characterizing selectively some nuclei across the major clusters, or superficial/lateral versus deep 

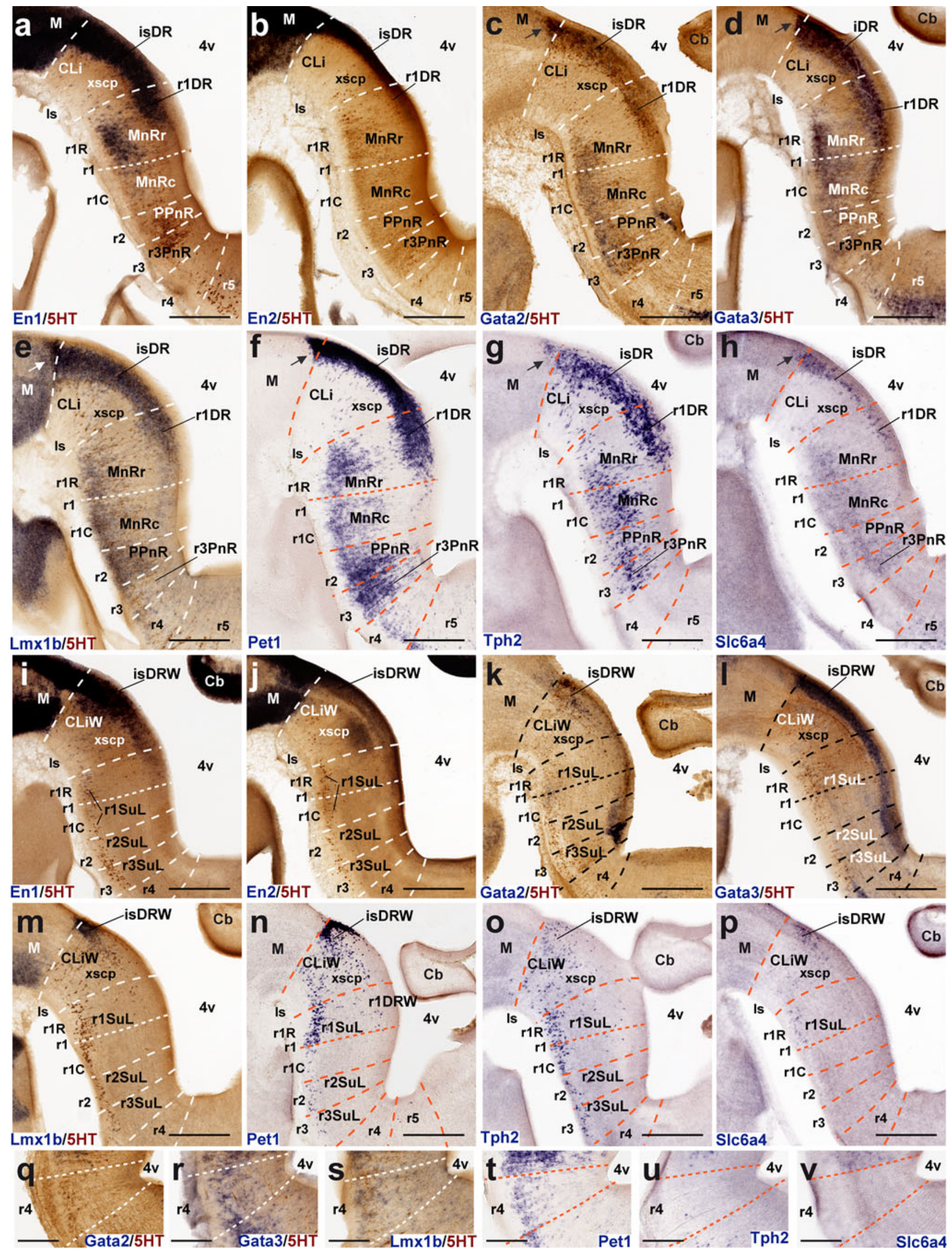
4Fig. 8 Expression of genes related with the serotonergic phenotype in the rostral raphe cluster in sagittal sections at E14.5. a-h Paramedian sections; the panels show 5-HT immunoreaction plus a particular in situ hybridization (a-e) or only an in situ hybridization (f-h). i-p More lateral section level; i-m show double 5-HT immunoreaction plus in situ hybridization, and $\mathbf{n}-\mathbf{p}$ only in situ hybridization. q-v Higher magnification of $r 4$ in six equivalent paramedian sections with 5-HT immunoreaction plus in situ hybridization $(\mathbf{q}-\mathbf{s})$, or only in situ hybridization $(\mathbf{t}-\mathbf{v})$. The riboprobes used are indicated in each case in blue color at the lower left $(\mathbf{a}-\mathbf{q})$ or lower right $(\mathbf{r}-\mathbf{v})$ corner. Arrows in $\mathbf{c}-\mathbf{h}$ point to the $\mathrm{mDR}$ nucleus. Scale bar $250 \mu \mathrm{m}$ in $\mathbf{a}-\mathbf{p}$, and $150 \mu \mathrm{m}$ in $\mathbf{q}-\mathbf{v}$

or intermediate paramedian serotonin cells across several rhombomeres. Here we show only a small selection of such genes, which indeed corroborate such differential molecular characteristics across the raphe system. Though the differential distribution is shown here optimally in paramedian sagittal sections, we corroborated in every case the implication that we deal with cells lying within true raphe nuclei in available coronal section material.

\section{Genes expressed in the rostral and caudal clusters}

We identified some examples of genes expressed in the two clusters, but restricted in both of them to specific cell subpopulations, clearly leaving other serotonin populations unlabeled: Cbln2 (cerebellin 2 precursor protein), Chrna7 (cholinergic receptor, nicotinic, alpha polypeptide 7), $\mathrm{Trh}$ (thyrotropin releasing hormone) and Zfhxlb (zinc finger homeobox $1 b$ gene).

Cbln2 is expressed in scattered mDR cells, separate ventral and dorsal subpopulations of isDR, fewer r1DRd/ r1DRv cells, abundant rostral and caudal MnR elements, and scattered PPnR cells, largely excluding other parts of the rostral cluster (Fig. 14a). There were only sparse pontine positive cells. In the caudal cluster, Cbln 2 is expressed only in scattered r6RMg and in ROb cells, the latter extending caudally as far as $\mathrm{r} 9$, but there is no signal in $\mathrm{RPa}$ or in r5RMg (Fig. 14a).

Chrna 7 is expressed rostrally densely and very selectively at the r1DRd subdivision (no isDR or $\mathrm{mDR}$ ) and in scattered $\mathrm{MnR}$ and PPnR cells, whereas caudally this gene is expressed in scattered cells at the RMgD and ROb, mainly across r5-r8 (Fig. 14b). R3 and r4 are devoid of any Chrna7 signal.

Trh shows an even more restricted expression pattern, with few cells selectively present in the $\mathrm{mDR}$, even fewer at the isDRd, and some detected at the $\mathrm{r} 1 \mathrm{SuL}$, whereas restricted cell patches decorate the r6RMgD, r7-r8RPa, and r10ROb (Fig. 14c), as well as the full PPy complex (not shown).

$Z f h x l b$ is selectively and densely expressed at the isDRd subdivision (no $\mathrm{mDR}$, and few r1DRd and r1DRv cells), as well as in scattered cells of the CLi, MnR, and PPnR. There are also some marked cells at the $\mathrm{RMgV}$ (r5 and r6) and r7RPa (Fig. 14d).
Genes expressed mainly in the rostral cluster

Hdac6 (histone deacetylase 6) is preferentially expressed in the mDR, isDRd and isDRv, though there is also some dispersed signal at the MnR and PPnR groups (Fig. 14e). Grm3 (glutamate receptor, metabotropic 3) labels strongly the inferior olive and basilar pons, and has weaker expression mainly restricted to the MnR, PPnR and PnR, although some positive cells appear as well at the $\mathrm{mDR}$ and isDR (Fig. 14f).

\section{Genes mainly expressed in the caudal cluster}

Given genes are expressed selectively at the RMg, or both $\mathrm{RMg}$ and ROb. For instance, Cart (cocaine and amphetamine regulated transcript) is expressed practically only in the r5-r6RMgD nuclei (Fig. 14g). In addition, Esrrb signal (estrogen related receptor, beta) is largely restricted to $\mathrm{r} 5$ r6RMgV (and nearby nucleus of the trapezoid body in r5), but appears as well in some PnR cells (Fig. 14h).

Other genes are medulla-selective. For instance, Lhx3 (LIM homeobox protein 3) and Npas1 (neuronal PAS domain protein 1) are two genes with a similar expression pattern restricted to intermediate paramedian serotonin cells of the caudal cluster. Both signals characterize $\mathrm{RMgD}$ in r5 and r6, as well as ROb in r7-r10 (Fig. 14i, j).

\section{Discussion}

We examined the hypothesis already introduced by Cambronero (1999), Puelles et al. (2007) and Jensen et al. (2008) that serotonergic neuronal populations occupying raphe nuclei of the mouse hindbrain have a distinct neuromeric distribution, as well as some neuromere-specific molecular characteristics. Though we have concentrated on serotonergic neurons, it is well known that other neuronal types coexist at many of the raphe nuclei, as defined cytoarchitectonically (review in Nieuwenhuys 1985). In the present report, we studied the postnatal spatial distribution of 5-HT-immunoreactive and Pet1-expressing cell bodies relative to rhombomeric landmarks and the differential expression patterns of several genes involved in serotonergic differentiation at given developmental stages. A number of points relative to our criteria for performing the segmental mappings were argued in "Results". Some extrapolation and, therefore, some inherent error, are implicit in our procedure. Our findings about a raphe segmental pattern in the mouse are largely consistent with similar earlier analyses done in the chick (Cambronero 1999; Puelles et al. 2007) or mouse (Jensen et al. 2008). The observed pattern is probably conserved at least in tetrapods, if not shared by all vertebrates (Nieuwenhuys 


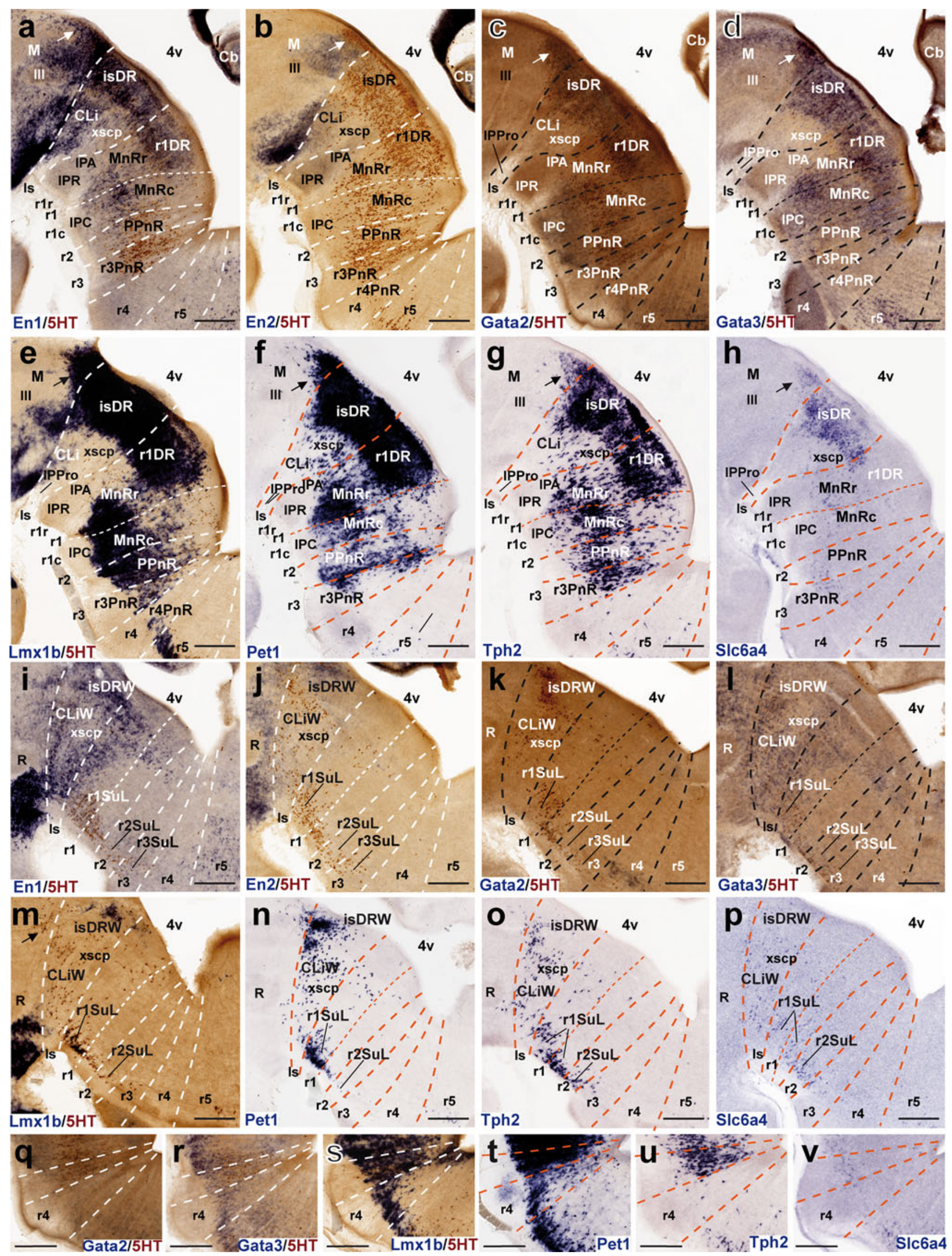


4Fig. 9 Expression of genes related with the serotonergic phenotype in the rostral raphe cluster in sagittal sections at E18.5. a-h Paramedian sections; the panels show 5-HT immunoreaction plus a particular in situ hybridization (a-e) or only an in situ hybridization (f-h). i-p More lateral section level; i-m show double 5-HT immunoreaction plus in situ hybridization, and $\mathbf{n}-\mathbf{p}$ only in situ hybridization. q-v Detail of $\mathrm{r} 4$ in six equivalent paramedian sections (not identical with a-h) with 5-HT immunoreaction plus in situ hybridization (q-s), or only in situ hybridization (t-v). The riboprobes used are indicated in each case in blue color at the lower left $(\mathbf{a}-\mathbf{q})$ or lower right $(\mathbf{r}-\mathbf{v})$ corner. Arrows in $\mathbf{a}-\mathbf{h}$ point to the $\mathrm{mDR}$ nucleus. Scale bar $250 \mu \mathrm{m}$

1985, 2009). In the following section we will compare this emerging segmental scenario of serotonergic raphe neuronal populations with previous models of this neuronal system, which uniformly assumed concepts now held to be obsolete about anteroposterior hindbrain boundaries. An additional source of confusion was the unfortunate convention to accept standard atlas coronal sections through rostral hindbrain as being transversal, when they really are horizontal (see Fig. 2o; e.g., the so-called dorsal raphe nucleus is actually a rostral structure). We will also examine how this map correlates with reported raphe heterogeneities regarding cellular morphology, neurochemical profile and patterns of connectivity. Conceptually, a segmental model of the raphe system leads us to expect heterogeneities, since rhombomeres have partially distinct molecular identities that might cause variant phenotypic aspects, as opposed to the common neurotransmitter phenotype, which is probably due to a set of shared genetic determinants.

Old and modern views on brainstem boundaries bear upon our topographic conception of the raphe nuclei

In general, available descriptions of the distribution of murine raphe nuclei are based on the traditional anatomic model of the midbrain and hindbrain regions in mammals, which is strongly laden with concepts taken from human brainstem anatomy. The basic subdivisions considered were the midbrain, the pons and the medulla, and, accordingly, raphe populations were assigned in mammals to mesencephalic, pontine or medullary territories (e.g., Taber et al. 1960; Dahlström and Fuxe 1964; Swanson 1992, 1998, 2003; Paxinos and Franklin 2007; Dong and The Allen Institute for Brain Science 2008; see Fig. 15b). This pons-dominated conception is now thought to need drastic corrections, due to its lack of a consistent fundament in developmental data (Puelles et al. 2007, 2012b; Watson and Paxinos 2010; Watson et al. 2010; Martínez et al. 2012; Watson 2012). Only rough anatomic criteria were available some 100 years ago for delimiting rostrally and caudally the adult pons (e.g., the ponto-mesencephalic and ponto-medullary surface sulci, due to relief of the pontocerebellar fibers of the middle cerebellar peduncle). Even these simple landmarks were liable to cause confusion, particularly when applying what is valid for human anatomy to rodent anatomy. Indeed, it passed generally unnoticed that in rodents all pontocerebellar fiber course rostral to the trigeminal root, so that there is no pontomedullary sulcus analogue; this situation changed in primates and other large mammals with further evolutionary development of the pons and the cerebellum (Nieuwenhuys 2001, 2009). Moreover, the mammalian basilar pons and its cerebellopetal fibers are essentially added superficial structures relative to the more primitive hindbrain tegmentum within (via evolution of the rhombic-lip-derived tangential pontine migration), so that the extrapolation of apparent pontine limits into the depth of the brainstem is not conceptually solid (or developmentally consistent). The simplistic subdivision of the hindbrain into pons and medulla was practical for the status of neuroanatomy 100 years ago, but was suspect already at that time; it essentially disregarded antecedent developmental data on a prepontine or isthmic component of the hindbrain, present also in humans (His 1893, 1895), as well as accrued data on hindbrain segmentation in all vertebrates (note that discovery of rhombomeres at the late nineteenth centurye.g., Orr 1887-preceded the discovery of raphe nuclei; see reviews in Vaage 1969, 1973; recent updates appear in Nieuwenhuys 2009, 2011; Watson 2012; Puelles 2012, in press).

These long-accepted, conventional 'pontine' limits for the human midbrain and medulla, which have been the basis for raphe nuclei classification, are no longer satisfactory from our present-day perspective, since they clearly do not agree either with the modern molecularly defined isthmo-mesencephalic border (e.g., Zervas et al. 2004; Jensen et al. 2008) and the related, now firmly established, critical patterning role of the isthmic organizer, nor with any of the developmental boundaries separating the now widely accepted rhombomeric histogenetic units.

The number of such units considered here may need comment. The overtly bulging r1-r6 units, or overt rhombomeres (delimited early on by outer constrictions), were long thought to be transient (and therefore unimportant for anatomic or functional explanatory purposes), but we now know by fate-mapping that they actually only become modified by thickening of their walls as the mantle layer differentiates, thus losing the limiting interrhombomeric constrictions, but their derivatives in the mantle maintain the primary intrinsic boundaries (fate maps by Marín and Puelles 1995; Wingate and Lumsden 1996), as well as the corresponding molecular coding differences (Marín et al. 2008). Other parts of the hindbrain found rostral and caudal to the set of overt rhombomeres never show transverse delimiting constrictions, but comparable cryptic (hidden) 

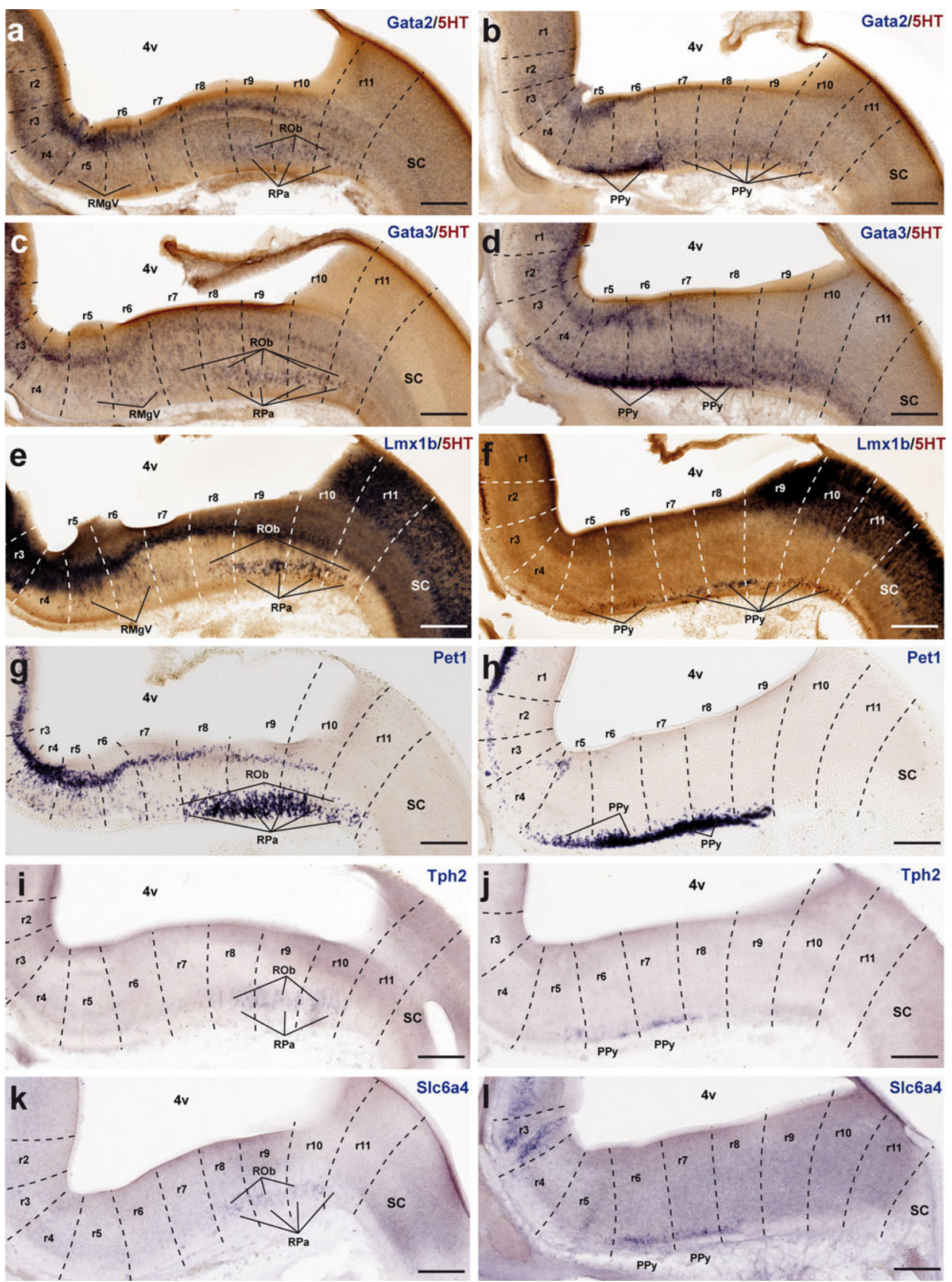
4Fig. 10 Expression of genes related with the serotonergic phenotype in the caudal raphe cluster in sagittal sections at E12.5. a-I Each pair of adjacent images represents paramedian and more lateral section levels reacted with a given probe and 5-HT immunoreaction (a-f) or only with an in situ probe (g-l). The relevant genes are indicated in blue color at the upper right corner of each photograph. Scale bar $250 \mu \mathrm{m}$

boundaries have been found, identifiable in the embryonic and mature hindbrain as fate limits and molecular limits; this is the case of the so-called crypto-rhombomeres Is and r7-r11 (Cambronero and Puelles 2000; Marín et al. 2008, Lorente-Cánovas et al. 2012). Note there are authors that do not distinguish Is from $\mathrm{r} 1$ proper (for instance, Jensen et al. 2008); they obviously allude as well to isthmic derivatives each time they refer to ' $\mathrm{r} 1$ '.

These results on the whole corroborate the idea that the causally important boundaries are the intrinsic ones, due to molecular patterning, since these condition differential histogenesis and anatomic structure. Surface constrictions are epiphenomena of morphogenesis, and may be visible counterparts of true molecular limits or not. From that point of view, crypto-rhombomeres are true rhombomeres. A further point in the background of our approach is the circumstance that the roots of the cranial nerves generally show invariant positions relative to the set of 12 rhombomeres, irrespective of the amount of axial bending that the hindbrain may suffer during morphogenesis. A preliminary discussion of the issue of anatomic discrepancies emerging with modern molecular analysis of hindbrain boundaries, and touching on the morphologic meaning of the cranial nerve roots, appeared in Rubenstein and Puelles (1992).

We will deal now with some current errors inherited from the recent 100 years of non-segmental neuroanatomy. This era began at the start of the 20th century with the discovery by Gaskell (1889) and Johnston (1902) of hindbrain columns; general enthusiasm about their functional importance had the effect of relegating apparently non-functional and supposedly transient rhombomeres to oblivion. Nevertheless, rhombomeres have returned as important patterning and histogenetic units with the molecular era, being remarkably consistent with multiple results from transgenic progeny tracing, mutated phenotypes and genoarchitectony.

A relevant point bearing on the classification of rostral raphe nuclei is the definition of the midbrain-hindbrain boundary (MHB; Zervas et al. 2004; see also Puelles et al. $2012 b$ ), since these formations have been systematically misclassified as being mesencephalic as a whole. The MHB lies at the midbrain-isthmus interface (originally identified by His 1893, 1895, and corroborated by Palmgren 1921 and Vaage 1969, 1973), where the isthmic organizer exerts a long-range inductive influence in both directions (the boundary itself is marked by apposed thin transverse rings of Wntl and Fgf8 expression, and by the apposition of wider fields of expression of Otx2 and Gbx2; HidalgoSánchez et al. 1999, 2005; Simeone 2000). These findings on rostral hindbrain patterning, which included the mechanism for the development of the cerebellum, first emerged in the late 1980s (Martinez and Alvarado-Mallart 1989). It is now strongly supported by a variety of experimental studies and several mouse mutant phenotypes, corroborating the existence and complexity of a sizeable rostral portion of the hindbrain that is essentially prepontine in developmental topography and causal background. A portion of the interpeduncular fossa and peduncles lying caudal to the oculomotor root, e.g., at the locus of the interpeduncular nucleus, as well as the whole pedunculopontine, isthmic and parabrachial areas, belong to the rostral hindbrain, and must be interpreted, together with the contained raphe nuclei, as Is, r1 and r2 derivatives. The midbrain accordingly does not contact the pons at all, and, as we have seen, only a minor rostral part of the dorsal raphe nucleus can be attributed to the caudal midbrain (Puelles et al. 2012b; Allen Developing Mouse Brain Atlas; http://www.developingmouse.brain-maps.org).

Note that the basilar pontine nuclei selectively aggregate within $\mathrm{r} 3$ and $\mathrm{r} 4$ after their tangential migration (they originate at the rhombic lip roughly at r6-r7 levels). Similarly, the transitional pontomedullary domain (r5-r6) separates the pons from the hindbrain medulla proper ( $\mathrm{r} 7-$ r11), most of which is characterized by the inferior olive. The basilar pontine nuclei in $\mathrm{r} 3-\mathrm{r} 4$ therefore can be precisely delimited developmentally from the set of prepontine hindbrain histogenetic units (isthmus, plus r1 and r2), as well as from the retropontine ones (r5-r11). This pattern is common to all vertebrates and should be the fundament of hindbrain anatomy.

It is also relevant to consider the developmental position of the cerebellum, due to the classic notion of a pontocerebellar developmental unit, which has turned out to be fictitious. The cerebellar vermis derives from the Is, and the cerebellar hemispheres and flocculus from r1 (see our Figs. 2, 3; review in Hallonet and Alvarado-Mallart 1997). In any case, the entire cerebellum clearly develops rostral to the pons (in $\mathrm{r} 3, \mathrm{r} 4$ ) in a dorsal prepontine hindbrain domain subject to the inductive influence of the isthmic organizer, whereas the pons proper develops outside of that domain. Therefore, the cerebellum is not a dorsal appendage of the pons other than topographically, irrespective of what is taught under traditional anatomic assumptions. Consequently, the middle cerebellar peduncle is not a transversal or ventrodorsal pathway. In the classic anatomic conception, the isthmus and extracerebellar r1 domains were wrongly included either in the pons or in the midbrain, and r2 jointly with the entire cerebellum was systematically assigned to the pons. This last error was probably caused by the fact that ponto-cerebellar fibers (all 
Fig. 11 Expression of genes related with the serotonergic phenotype in the caudal raphe cluster in sagittal sections at E14.5. a-I Each pair of adjacent images represents paramedian and more lateral section levels reacted with a given probe and 5-HT immunoreaction (a-f) or only with an in situ probe $(\mathbf{g}-\mathbf{l})$. The relevant genes are indicated in blue color at the upper right corner of each photograph. Scale bar $250 \mu \mathrm{m}$
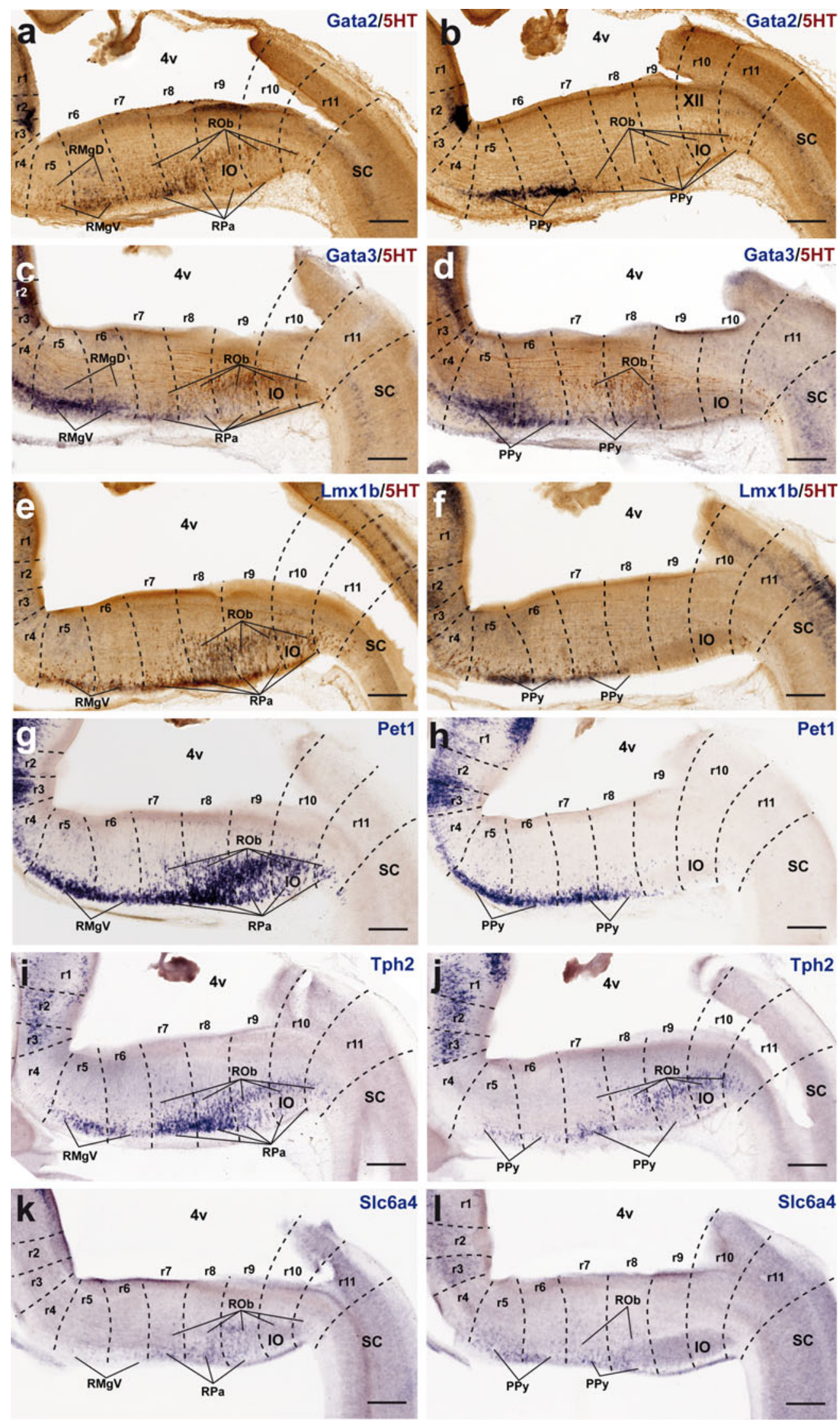
Fig. 12 Expression of genes related with the serotonergic phenotype in the caudal raphe cluster in sagittal sections at E18.5. a-l Each pair of adjacent images represents paramedian and more lateral section levels reacted with a given probe and 5-HT immunoreaction (a-f) or only with an in situ probe $(\mathbf{g}-\mathbf{l})$. The relevant genes are indicated in blue color at the upper right corner of each photograph. Scale bar $300 \mu \mathrm{m}$
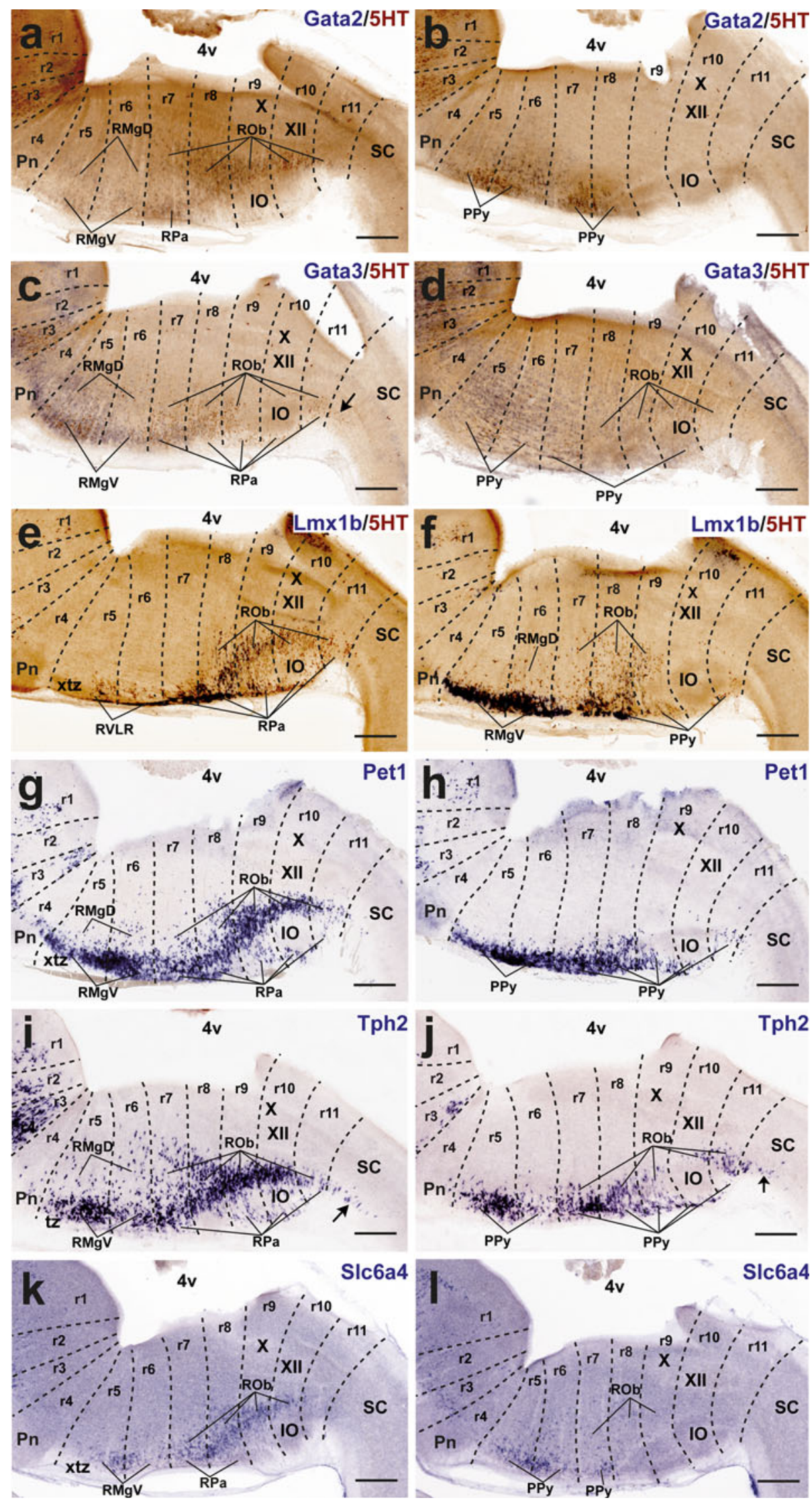
a

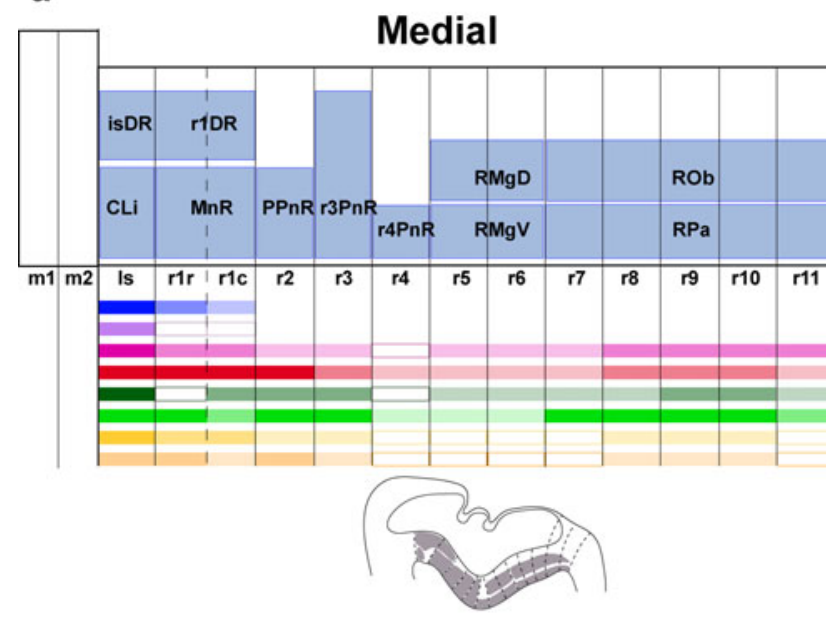

C

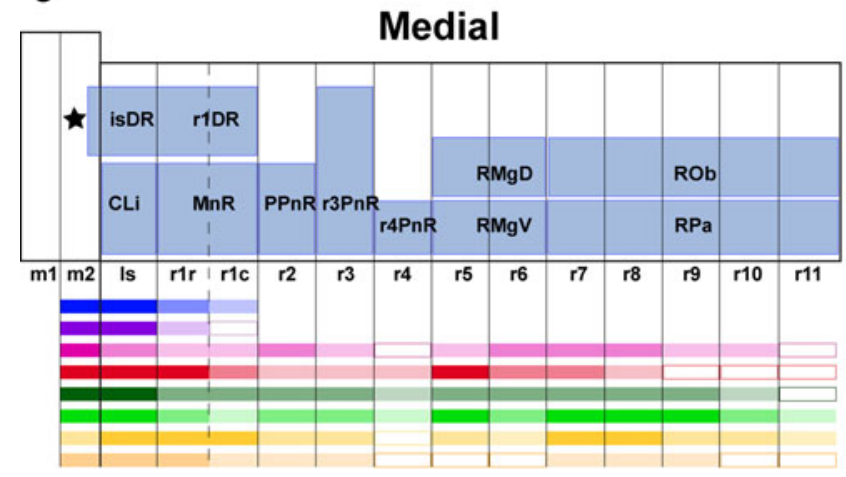

e

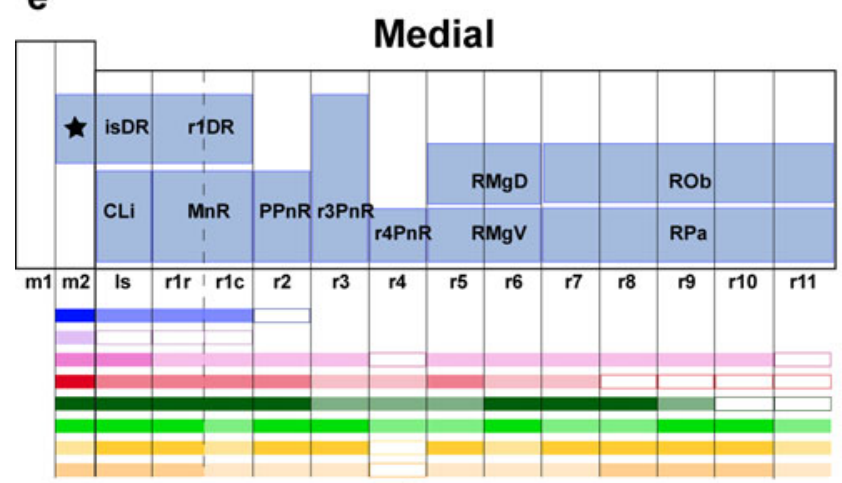

b

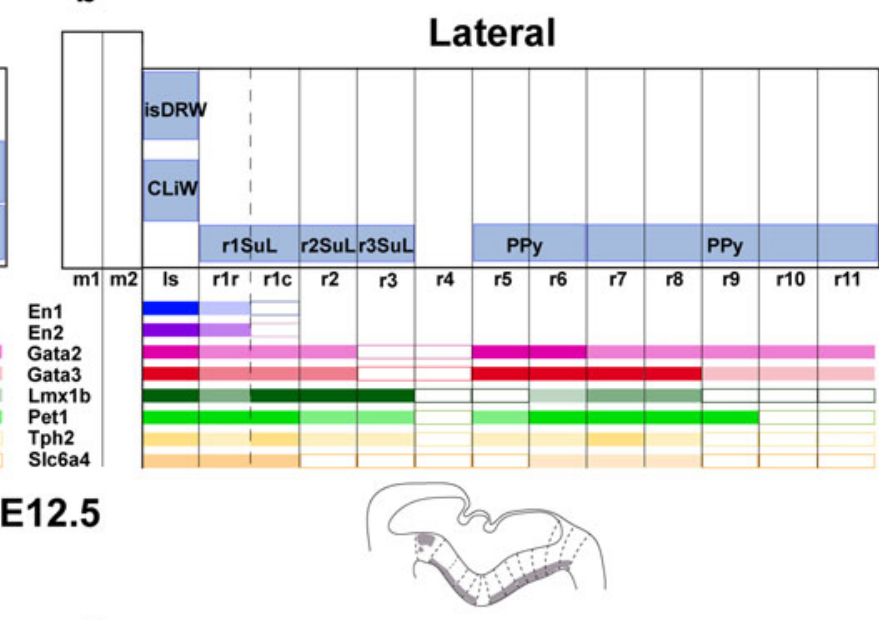

d

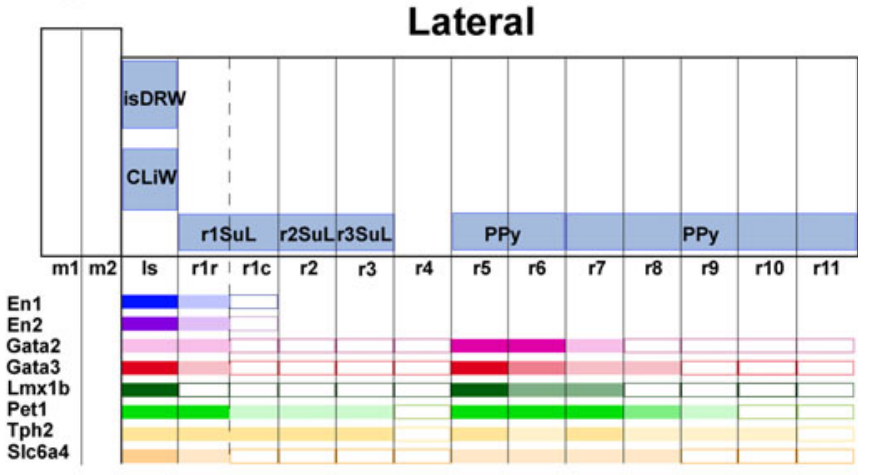

E14.5
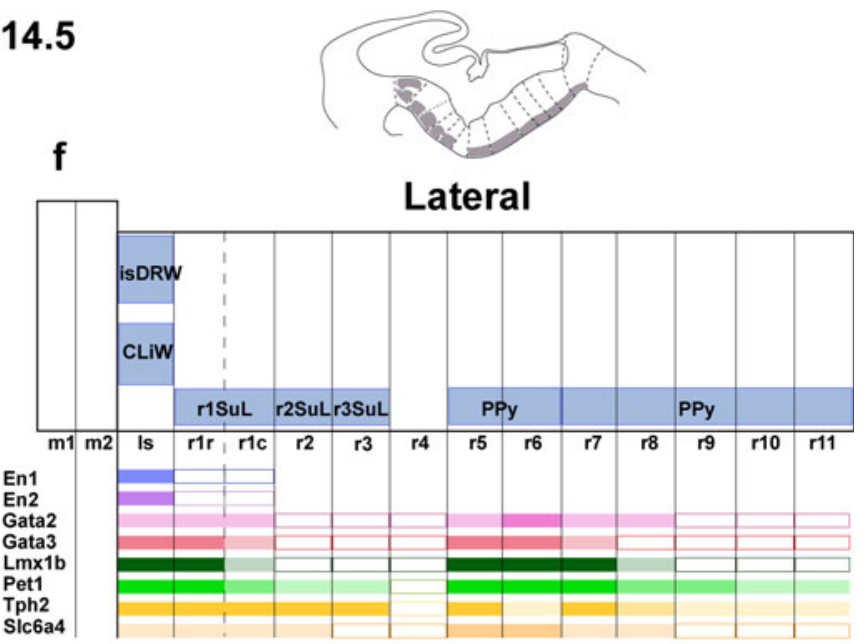

E18.5

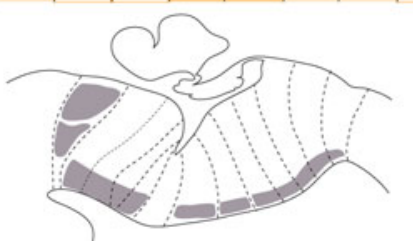

hindbrain, with mapped raphe populations filled-in in gray, the respective intensity of gene expression for eight color-coded markers is represented by different color hue. Underneath is added a realistic schema of the corresponding sagittal section thus mapped. Black asterisks in $\mathbf{c}$ and $\mathbf{e}$ represent the expanding $\mathrm{mDR}$ nucleus 
Fig. 14 Selected results of a search done in the Allen Adult and Developing Mouse Brain Atlases, looking for mouse genes with restricted expression patterns within the raphe nuclei (irrespective of other domains of expression). The gene tag is indicated at the lower left corner of each panel. a-d Genes with expression restricted to some specific raphe subgroups of the rostral and caudal clusters. e,

f Genes with expression

restricted to only some nuclei of the rostral cluster. $\mathbf{g}-\mathbf{j}$ Genes with expression restricted to only some nuclei of the caudal cluster. The stages are P4 (d, f, $\mathbf{h}, \mathbf{i}, \mathbf{j})$, P14 (a-c) or P56 (e, g). Scale bar $500 \mu \mathrm{m}$
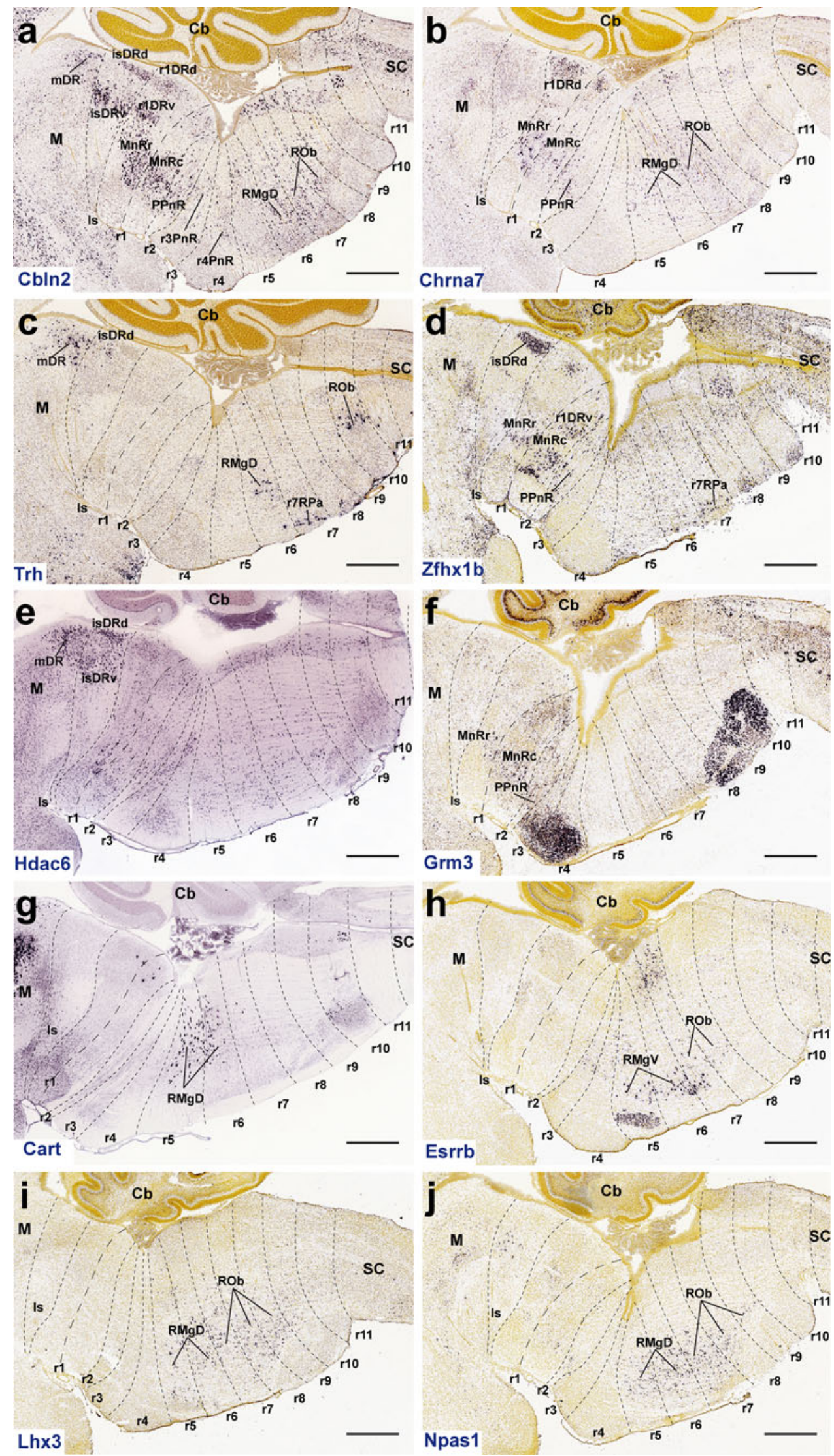
Fig. 15 Comparison of old and new raphe classifications. a Schematic median projection of paramedian raphe serotonergic cells taken from a postnatal mouse specimen, showing relative cell densities. b Schema of the conventional identification of 9 paramedian raphe nuclei across midbrain, pontine and medullary territories, the latter delimited roughly according to the apparent external bulge of the pons; the thick red lines mark approximately the postulated 'pontine' boundaries, though there is some variation between sources (compare text Fig. 10 in Swanson 1998; text Fig. 0 in Paxinos and Franklin 2007; see also Dong and The Allen Institute for Brain Science 2008). c Schema illustrating present results, ascribing 25 paramedian serotonergic populations to discrete neuromeric origins. The limits of the midbrain $(M)$, developmental hindbrain $(H)$ units containing the basilar pons (pons proper) and the spinal cord $(S C)$ are marked in red. Laterally displaced cell groups are not represented in this panel

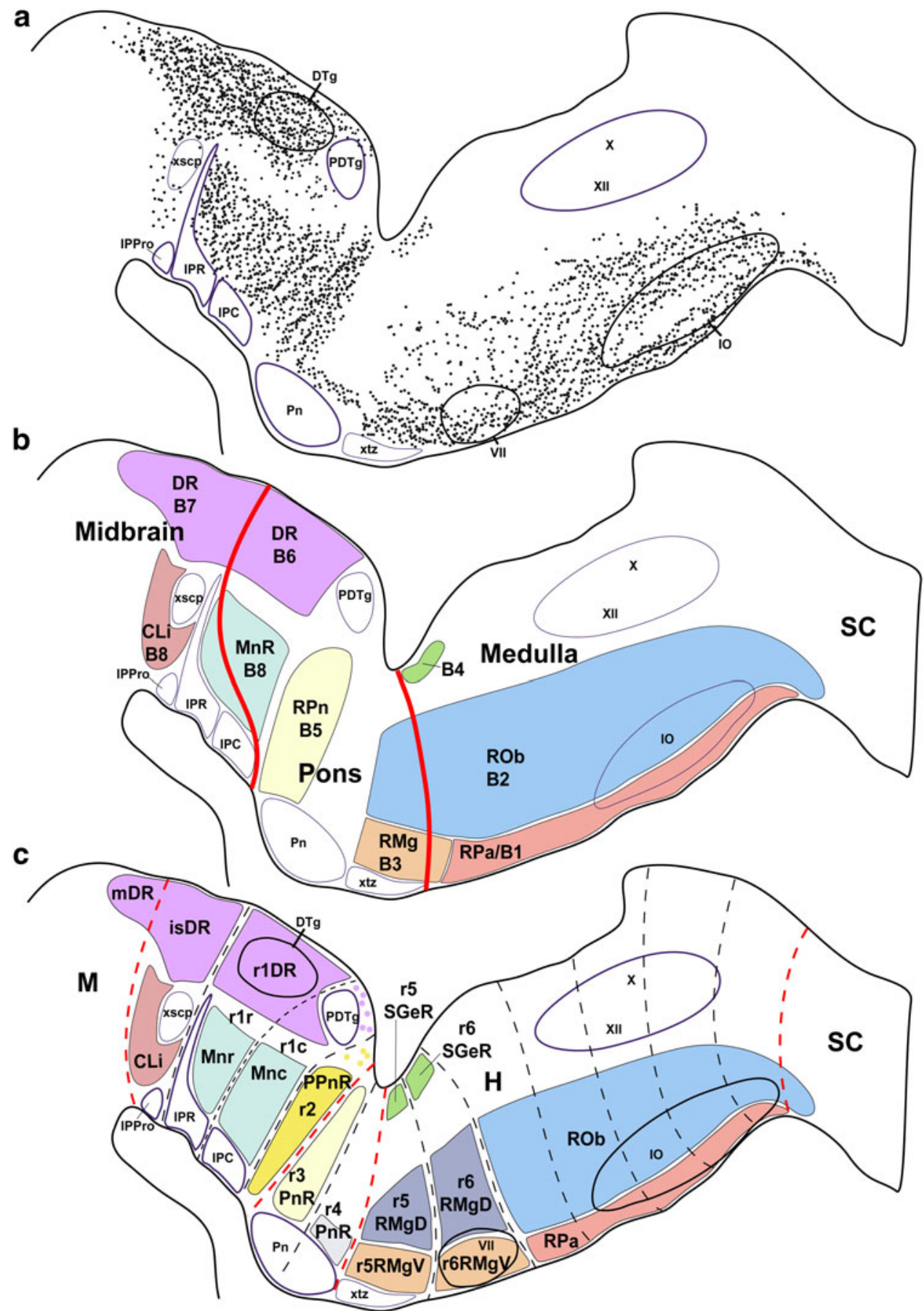

of them in rodents and most small mammals) course into the cerebellum via $\mathrm{r} 2$ and $\mathrm{r} 1$, passing first longitudinally rostral to the trigeminal root, which always enters the brainstem at the caudal end of $\mathrm{r} 2$, before bending into the cerebellum. Only primates, cetacea and other large mammals have ponto-cerebellar fibers coursing behind the trigeminal root (Nieuwenhuys 2001, 2009). As a result, the trigeminal root is widely described as entering the 'pons', although sectioned material will show that this root only relates to the brachium pontis, rather than to the pontine nuclei. Unfortunately, it was not thought necessary classically to distinguish the 'pons' sensu stricto, that is, the basilar pontine nuclei in r3-r4, from the 'pons' sensu lato, which includes the pontocerebellar fibers, coursing via r2 into 1 (primitively) or also via r 3 and $\mathrm{r} 4$ into $\mathrm{r} 2$ and then $\mathrm{r} 1$ (in primates and large mammals).

The modern pontomedullary boundary (PMB), defined just caudal to the basilar pontine nuclei, lies between $\mathrm{r} 4$ and r5, ventrally separating macroscopically the basilar pons from the trapezoid body (Fig. 15c). This contrasts with the classical concept, in which part of the retropontine r5 and 
r6 developmental units (containing the abducens and the migrated facial motor nuclei, as well as the trapezoid body and superior olivary complexes), were wrongly assigned to the 'pons', at least in human neuroanatomy textbooks. The raphe magnus nucleus falls into this domain. This error apparently was due to the ventral bulge of the much deformed $\mathrm{r} 4$ basilar pontine region in the human brainstem, which sags like an apron over the ventral surface of $r 5$ and $r 6$ (this does not occur in rodents; Nieuwenhuys 2001, 2009). Notably, the abducens nerve arising at $\mathrm{r} 5$ level always emerges freely from under the pons (Nieuwenhuys 2001). The caudalmost cerebellopetal pontine fibers, being oriented toward the cerebellum in $\mathrm{r} 1$, do not cover the dorsalmost parts of $\mathrm{r} 2-\mathrm{r} 4$. This is demonstrated by the fact that the facial and cochleo-vestibular nerve roots, which penetrate the hindbrain through $\mathrm{r} 4$, and therefore are proper pontine nerves, do not traverse the pontocerebellar fibers, but enter the free pial surface of the $\mathrm{r} 4$ alar plate dorsocaudal to the brachium pontis; similarly, the cochlear nuclei formed next to the rhombic lip in r2-r5 lie at the brain surface, free of pontine fibers.

This developmental topologic analysis leads to the conclusion that the two macroscopic sulcal boundaries of the pons, which delimit the middle cerebellar peduncle, are not really transversal anatomic boundaries relative to the true developmental units, the rhombomeres, and even are not constant in mammals. Accordingly, they do not coincide with the causally relevant transversal neuromeric molecular boundaries. Moreover, the midbrain is separated from the pons by a sizeable prepontine hindbrain domain (Fig. 15c; this conception is represented in the Allen Developing Mouse Brain Atlas). Modern molecular and causal understanding of hindbrain structure thus requires us to downplay the relative importance of the classic pons concept, and we have to accept the caudal limit of the midbrain in front of the isthmus, the prepontine nature of cerebellum, isthmus, $\mathrm{r} 1$, and $\mathrm{r} 2$, and a retropontine pontomedullary region (r5-r6) that holds the trapezoid body and the facial motor nucleus. The medulla concept itself would be restricted to $\mathrm{r} 7-\mathrm{r} 11$, wherein the inferior olive appears at r8-r11 levels. The rhombo-spinal boundary lies developmentally across the fifth somite (fate mapping by Cambronero and Puelles, 2000), which corresponds to a plane just caudal to the pyramidal decussation (in r11).

The conserved topography of the entrance points and intraneural courses of the cranial nerve roots relative to rhombomeres in all vertebrates (Vaage 1969; Nieuwenhuys 1998, 2009) provides the strongest help for recognizing in sagittal and horizontal sections the mature derivatives of the relevant hindbrain histogenetic fields, as has been corroborated by a number of modern experimental or transgenic fate-mapping studies (e.g., Carpenter et al. 1993; Marín and Puelles 1995; Studer et al. 1996; Gavalas et al. 1997; Schneider-Maunoury et al. 1998; Cambronero and Puelles 2000; Oury et al. 2006; Tümpel et al. 2009). This is the novel background for our segmental analysis of the raphe nuclei.

Neuromeric topography and classification of the raphe nuclei

Complementarily to the partial and somewhat simplistic treatment offered by Jensen et al. (2008), our present map of raphe nuclei redresses the mentioned descriptive inaccuracies due to the traditional anatomic model, by taking into account the topological relations of all serotonergic neuronal populations found within the 12 rhombomerederived domains (isthmus plus r1-r11), thus offering for the first time a coherent full explanation of their evident heterogeneity along the longitudinal axis (Figs. 1 and 15). Beyond the existence of 12 separate rhombomeric origins, the variety of raphe populations is increased by the fact that serotonergic neurons developing within each rhombomere may adopt diverse stereotypic radial and/or medio-lateral positions within the basal plate. Note that, irrespective of their collective name identifying them as 'raphe' formations, none of the studied cell groups originates from the raphe proper, which is a $S h h$-positive median astroglial palisade formed at the hindbrain floor plate. Serotonergic raphe neurons are generated instead from an adjacent $N k x 2.2$-positive strip (formed in response to local strong Shh signaling; Shimamura et al. 1995), which constitutes the paramedian or ventralmost microzone of the hindbrain basal plate (Briscoe et al. 1999). The raphe populations normally migrate radially into the local mantle, and differentiate there, occupying, therefore, a paramedian position adjacent to the glial raphe, either periventricularly or within the intermediate or superficial strata. Secondary migratory invasion of the median raphe territory by some serotonergic neurons occurs at later developmental stages, particularly in the dorsal raphe complex. Other raphe populations migrate in the opposed, lateral direction, reaching slightly more lateral positions at any of the three strata. A majority of these laterally displaced cells aggregate superficially (e.g., PPy cells), though others are secondarily separated from the pial surface by some later developing structure (usually tracts, or the basilar pons); the latter cells are found postnatally in ventrolateral parts of the basal plate territory (e.g., the SuL cells; Lidov and Molliver 1982; Wallace and Lauder 1983; Goto and Sano 1984).

Based on the tridimensional distribution of serotonergic neurons, we conclude that there exist at least forty-five distinct raphe nuclei, leaving apart dispersed cells inbetween. Since often there are similarities in their radial and mediolateral location across some neighboring rhombomeres (possible shared guidance mechanisms, or 
adhesive properties), the individual periventricular, intermediate, lateral and ventrolateral (superficial) nuclei can be grouped into a number of plurineuromeric nuclear complexes, which largely correspond to the serotonergic cell groups conventionally recognized in the classic and alphanumeric terminologies (Table 1). From a developmental perspective, rostral (prepontine and pontine) and caudal (pontomedullary and medullary) developmental clusters were already identified previously (Olson and Seiger 1972; Seiger and Olson 1973; Lidov and Molliver 1982; Wallace and Lauder 1983; Goto and Sano 1984).

The segmental character of the rostral cluster was revealed by Jensen et al. (2008); these authors used sophisticated intersectional and subtractive genetic fatemapping tools to investigate postnatal serotonergic cells (marked by expression of Pet1) that were derived, respectively, from what the authors identified as ' $\mathrm{r} 1$ ' (the sum of Is and r1; selected by the co-expression of Enl in this territory), r2 (by co-expression of Rse2) or r3 plus r5 (by co-expression of Egr2). The labeled rostral hindbrain raphe domains identified in this way essentially correspond to our results, particularly in underlining the contribution of Is and $\mathrm{r} 1$ (not distinguished in this study) to the DR complex (B7, B6), but jointly also to the rostral MnR (part of B8) and SuL elements (part of B9). The existence of a mDR component was not identified (the ' $\mathrm{r} 1$ ' labeling via Enl could not have distinguished the possible difference between midbrain versus isthmic origins, in any case). Our present analysis goes one step further in distinguishing midbrain, isthmic and $\mathrm{r} 1$ parts within the ' $\mathrm{r} 1$ '-derived complex, allowing finer analysis of the DR complex (needed, as we showed, by some available genoarchitectonic labeling patterns). Other populations that had conventionally been attributed to caudal parts of B8 and B9, were shown to be derived from $\mathrm{r} 2$ and $\mathrm{r} 3$; these clearly include our caudal MnR, PPnR and r3PnR cell groups, as well as the corresponding $\mathrm{SuL}$ groups (the pontine nature of some of these neurons was not mentioned). The serotonergic derivatives of $\mathrm{r} 5$ were deduced to include the $\mathrm{RMg}$ (its rostral half, according to us; see also Bang et al. 2012). No serotonergic cells were assumed a priori to derive from r4 (a dogma in the literature), and, consequently, it was not determined whether any pontine serotonergic cells were left unlabeled by either $\mathrm{r} 3$ or $\mathrm{r} 5$ fate mapping. The sketched median projection of segmental raphe populations offered by these authors (in their Figure 2a) is remarkable by the implied enormous size of the $\mathrm{r} 4$ domain, compared for instance with $r 2, r 3$ and $r 5$ (compare $r 3$ versus $r 4$ in our Fig. 1c). This may be due to artistic license. Possibly similar data shown on sagittal sections would have demonstrated even closer correspondence with our mappings. An important point made by Jensen et al. (2008) is that there is some intermixing of raphe cells derived from adjacent rhombomeres (corroborating similar general conclusions of Marín and Puelles 1995; Wingate and Lumsden 1996; Cambronero and Puelles 2000; Marín et al. 2008). Finally, Jensen et al. (2008) also concluded that some of the ' $\mathrm{r}$ ' ' derivatives aggregate caudally at the supragenual or B4 cell group; this would necessarily imply a tangential migration of ' $r 1$ ' elements into $r 5-r 6$, for which we did not see any support in our developmental analysis. We think that this discrepancy probably can be explained by assuming that their supposed B4 cells actually represent the sparser r1DRv cells found by us (also by Hale and Lowry 2011) periventricularly at caudal $\mathrm{r} 1$ level (that is, they would need to be interpreted as caudal B6, rather than B4; this would eliminate the need of conjecturing a very odd migration); the relevant illustrated material in cross sections in Jensen et al.'s (2008) Figure 2 does not show convincingly the genu of the facial nerve, which is a required landmark for the supragenual B4 cells.

Rhombomeric groupings are useful for the purpose of simplifying the terminology, but do not presuppose functional identity of the individual segmental components (the contrary is true, since different segmental origins, involving varying molecular identities, raise the possibility of subtle structural differentiations and corresponding functional consequences). We will discuss below some hodological peculiarities. Accordingly, we hold that any proposal of functional unity across a plurineuromeric raphe aggregate would need to be demonstrated experimentally, irrespective of the superficial anatomic similarity. The latter may be due merely to shared cell-positioning mechanisms.

In our segmentally adapted nomenclature, we tried to conserve as far as possible the conventional denominations of the raphe nuclear complexes (e.g., DR, ROb, RPa; Olszewski and Baxter 1954; RMg found in Taber et al. 1960; MnR found in Dahlström and Fuxe 1964; the same approach can be applied to the B group names of the alternative alpha-numeric terminology; see Fig. 15 and Table 1). The names 'supralemniscal' and 'parapyramidal' used by us are already found in the literature (Table 1). We followed the logic that apparent plurineuromeric sharing of radial and mediolateral topography across several rhombomeres is due to similar histogenetic mechanisms, irrespective of potentially variant individual molecular identities of their segmental units; a shared name thus seems also apposite.

\section{The rostral cluster}

The classic rostral cluster is essentially isthmic, prepontine and pontine, and contains diverse parts of the dorsal (DR), median $(\mathrm{MnR})$, prepontine $(\mathrm{PPnR})$ and pontine $(\mathrm{PnR})$ raphe nuclei, apart supralemniscal ventrolaterally placed 
cells (Puelles et al. 2007; Jensen et al. 2008; present results). We should remember that, developmentally, the mesencephalic, isthmic and $\mathrm{r} 1$ elements arise within the area of influence of the isthmic organizer, whereas the prepontine and pontine ones lie outside it.

We found that the hindbrain DR complex lies mainly across the isthmus and rostral half of $\mathrm{r}$, with a tardive minor extension into the caudal or preisthmic midbrain (m2). We deal separately below with the mesencephalic component of the DR complex. Remarkably, the entire DR complex, or most of it, is conventionally wrongly thought to be mesencephalic, due to the historic reasons sketched above (e.g., Swanson 1992; Paxinos and Franklin 2007; Dong and The Allen Institute for Brain Science 2008), while Jensen et al. (2008) and Bang et al. (2012) interpret it entirely as rhombencephalic. This point was specifically reexamined here by comparison of the developing DR with the selective midbrain marker Otx2. The latter expression domain clearly stops just in front of the DR complex up to E14.5, when the minor midbrain component starts to appear.

There are morphological and neurochemical antecedents of the $\mathrm{m} 2$, isthmic and $\mathrm{r} 1$ segmental subdivisions of the DR deduced by us. Several authors recognized three anteroposterior parts of the DR, based on distinctive patterns of cellular distribution and morphology (Dahlström and Fuxe 1964; Daszuta and Portalier 1985; Ishimura et al. 1988; Eaton et al. 1993; Abrams et al. 2004; Fu et al. 2010): the rostral and caudal portions (our mDR and r1DR, respectively) were found to be restricted to the midline, while the intermediate portion typically shows wing-like lateral expansions (our isDR with its lateral 'wings'; Hale and Lowry 2011). Topographic mappings of neurotransmitters and neuropeptides in the DR nuclear complex are also consistent with our three subdivisions: TH (tyrosine hydroxylase), somatostatin and CCK-(cholecystokinin) expressing neurons are present in the mDR (van der Kooy et al. 1981; Vanderhaeghen 1985; Priestley et al. 1993; Smith et al. 1994; Araneda et al. 1999; Fu et al. 2010; Puelles et al. 2012b). The isDR selectively contains a cell population positive for GAD67 (Fu et al. 2010), and enkephalin, NOS (nitric oxide synthase) and CRF-(corticotropin-releasing factor) expressing neurons have been detected in the r1DR (Merchenthaler 1984; Sakanaka et al. 1987; Commons and Valentino 2002; Fu et al. 2010).

Antecedents of segmental subdivisions of the other rostral cluster nuclei that appear at paramedian or lateral intermediate positions are less evident in the literature (apart Jensen et al. 2008); in fact, relevant data are scarce and confusing, largely because the longitudinal axis across prepontine areas tended to be interpreted conventionally as a dorsoventral dimension in cross sections (see Fig. 2o). Moreover, there is little agreement on the boundaries between individual intermediate raphe subpopulations, due to the confused view that they all lie in the caudal 'pedunculopontine midbrain'. Most authors assign all radially intermediate serotonergic populations lying in the neighborhood of the interpeduncular nucleus and the pons to the MnR (central superior) nuclear complex (e.g., Dahlström and Fuxe 1964; Törk 1990; Harding et al. 2004). In contrast, our map suggests that the serotonergic populations present in this intermediate paramedian region belong to four separate nuclear groups: CLi (a sparse population), MnR, PPnR and r3PnR, which belong to Is, r1, r2 and r3, respectively. Some earlier morphological and neurochemical data on these neurons is consistent with such subdivisions, including differences of their respective dendritic morphology and spatial distribution (see also Hale and Lowry, 2011). Dendrites parallel to the midline are typical in CLi (isthmus), whereas multipolar dendritic arbors were found in what we identify as MnR (r1) and PPnR (r2), and plexiform dendritic arrangements characterize the r3PnR cell population (Törk and Hornung 1990; Harding et al. 2004). A particular substance $P$ receptor profile is found across these rostral intermediate raphe populations: the neurokinin receptor $1(\mathrm{nkr} 1)$ is expressed selectively in CLi (Is) and r3PnR, whereas the neurokinin receptor 3 (nkr3) appears selectively in MnR (r1), and both of them are present at the PPnR in r2 (Léger et al. 2002).

The laterally migrated populations of the rostral raphe cluster (CLiW, r1-r3SuL, plus some r1DRW elements), which we found are distributed across Is, r1, r2 and r3, were described conventionally as dispersed serotonin neurons belonging to the 'reticular formation' (e.g., Vertes and Crane 1997; Hornung 2003); alternatively, they were lumped under the concept of 'supralemniscal nucleus' (Törk 1990; Jacobs and Azmitia 1992; Vertes and Crane 1997), or the 'B9 group' (Dahlström and Fuxe 1964). Works using immunohistochemical mapping, rather than the fluorescence methods, have emphasized the importance of these lateral serotonergic populations in rodents, in terms of the number of neurons and the longitudinal extent of their distribution (e.g., Vertes and Crane 1997). We propose that these raphe populations probably separate, respectively, from individual paramedian raphe formations in a plurineuromeric pattern: the CLiW emerges from the paramedian CLi (isthmus), the r1SuL, across both parts of r1, separates from the MnR (r1r, r1c), the r2SuL arises from the PPnR (r2) and the r3SuL spreads out from the r3PnR. We could not identify an equivalent lateral population in $\mathrm{r} 4$ (i.e., lateral to $\mathrm{r} 4 \mathrm{PnR}$ ).

A nucleus comparable to the $\mathrm{CLiW}$ is described by Puelles et al. (2007) in the chick-the so-called 'CLi alar process' (CLiA) - but such a concept is not found elsewhere in the literature on mammalian raphe cells. Instead, some authors assigned two lateral populations to a raphe nucleus called pontis oralis (PnO) (Jacobs et al. 1984; 
Azmitia and Gannon 1986; Hornung and Fritschy 1988; Törk and Hornung 1990). The rostral component of this $\mathrm{PnO}$ is located at the same place than our CLiW (isthmus); whereas the caudal $\mathrm{PnO}$ component seems to correspond to our r1SuL. According to our present rationale, none of these $\mathrm{PnO}$ entities is properly pontine, and, therefore, this name is misleading. This accounts for our proposal of new descriptive names (CLiW, r1SuL). We support adding the $\mathrm{CLiW}$ serotonergic population as a new nucleus belonging to the Is, distinct from the CLi.

Another aspect to discuss with regard to the ventrolateral SuL serotonergic nuclei refers to the reasons of their lateral situation relative to the medial, paramedian, or 'authentic' raphe nuclei. A tentative explanation of their position was offered by Steinbusch and Nieuwenhuys (1983), saying they contain " ... neurons that during ontogenesis did not complete their migration toward the raphe region". This implies the hypothesis that raphe neurons normally migrate into a paramedian position out of a more lateral (dorsal) origin, as was apparently first speculated by Harkmark (1954). Interestingly, Swanson (1992, 1993) represented all raphe nuclei in his flat brain map within a ventral part of the alar plate, indicating in the legend that some brainstem formations are mapped according to their developmental origin, rather than their adult position. Incidentally, these flat maps assign the CLi, DR and CS (or $\mathrm{MnR}$ ) raphe nuclei, jointly with a handful of other prepontine elements, to the midbrain. The evidence supporting the mapped alar origin of raphe nuclei was not identified expressly, though perhaps fate-mapping observations of Tan and LeDouarin (1991) were considered relevant; these authors found a few labeled raphe cells after quail-chick homotopic grafting of dorsal (alar) parts of the hindbrain (note that in those studies the neurotransmitter phenotype of such 'raphe' cells was not determined). Apart serotonergic neurons, up to 10 different sorts of neurotransmitteridentified neuronal cell types have been found in variable numbers within the classic raphe nuclei (Nieuwenhuys 1985; Hale and Lowry 2011). It is certainly possible that some alar derivatives of $\mathrm{r} 1$, in particular, approach by tangential migration the raphe neighborhood, but without representing a serotonergic population; this $\mathrm{r} 1$ migration was studied by Lorente-Cánovas et al. (2012), and they specifically excluded the serotonergic phenotype among the migrated derivatives.

In any case, now we know that, with exception of the DR complex and some medullary paramedian elements (Jensen et al. 2008), Nkx2.2 gene function is necessary for the development of the serotonergic phenotype in a rhombencephalic progenitor context; this condition only obtains in the paramedian basal plate adjacent to the hindbrain floor plate, due to the dependence of $N k x 2.2$ induction from the floor plate source of Shh morphogen
(Briscoe et al. 1999). From this point of view, we can safely assume that any laterally placed serotonergic neurons probably originated in the standard paramedian basal locus, and must have migrated afterwards to a more lateral deep, intermediate or superficial radial position within the basal plate, thus separating actively from the midline. This differential behavior suggests peculiar adhesive properties of these cells, which must be lacking in the cognates that remain at paramedian loci. We will mention below some molecular differences apparent between medial and lateral raphe populations.

A subdivision of the $\mathrm{MnR}$ and $\mathrm{r} 1 \mathrm{SuL}$ serotonergic populations-both in $\mathrm{r}$ - -into rostral and caudal subnuclei can be envisioned. This extra-large rhombomere uniquely has differential morphological and molecular characteristics in its rostral and caudal portions (e.g., Otx2 is expressed differentially only at the caudal $\mathrm{r} 1$ ), and several of the respective neuronal populations are somehow different (Lorente-Cánovas et al. 2012). Note for instance the clear restriction of the DTg/VTg and PDTg periventricular nuclei to $\mathrm{r} 1 \mathrm{r}$ and r1c, respectively (Fig. 15). Vaage (1969, 1973) already proposed that the $\mathrm{r} 1$ domain actually should be subdivided into two neuromeres, similar to our present r1r, r1c (review in Aroca and Puelles 2005), though this idea has failed to receive general support so far, roughly for the same reason that some authors abstain from separating the isthmus from ' $\mathrm{rl}$ ' (the cryptic nature of the proposed boundaries). Irrespective of how we classify it, the bipartite pattern of $\mathrm{r} 1$ clearly affects also the relevant raphe nuclei (present data) and neighboring tegmental nuclei, similarly as the underlying interpeduncular nucleus (Lorente-Cánovas et al. 2012). For instance, we noted that expression of Enl is distinctly stronger at the rostral part of MnR and r1SuL than at their caudal part; Pet1 is also stronger in $\mathrm{MnRr}$ than MnRc at E14.5 and E18.5, and Lmxlb shows a retarded upregulation at the $\mathrm{MnRr}$ (where it is selectively absent at E12.5), compared with MnRc.

\section{The caudal cluster}

The segmental organization of the raphe groups developing out of the caudal cluster-RMg, r5/r6PPy, SGeR, ROb, $\mathrm{RPa}$, medullary $\mathrm{PPy}$ - is more controversial, due to ambiguities in the conventional anatomic description of the individual formations. The RMg initially was called 'central inferior raphe nucleus' by Olszewski and Baxter (1954), presumably by comparison with the 'central superior nucleus', or MnR (note here objectionable use of 'superior-inferior' terms, referring to the rostral-caudal axial dimension). Both Lidov and Molliver (1982) and Törk and Hornung (1990) placed the RMg approximately 'between the caudal quarter of the pons and the rostral end of the inferior olive' (which would translate into $\mathrm{r} 4-\mathrm{r} 7$, in 
our terms). Developmentally, the pons proper (pontine nuclei) ends at the caudal limit of $r 4$; the r5 domain is well characterized by containing the trapezoid body, the superior olivary/periolivary complex and the abducens nucleus, whereas r6 selectively receives the migrated facial motor nucleus. The retrofacial part of the ambiguus motor nucleus characterizes the $\mathrm{r} 7$ region. The inferior olive ends rostrally somewhere in $\mathrm{r} 8$ and is fully absent in $\mathrm{r} 7$ (Marín and Puelles 1995). We interpreted that the territory referred to in the cited $\mathrm{RMg}$ description probably corresponded actually to r5-r7, since r4 shows very few raphe cells (see below), and we know that the pons used to be extended conventionally at least into the area we identify as r5. The rostralmost RMg cells (accurately labeled experimentally as r5-derived by Jensen et al. 2008) are otherwise described as coinciding with the trapezoid body (Hornung and Fritschy 1988), at section levels through the facial genu and the abducens nucleus (Törk and Hornung 1990), or the superior olive (Jacobs and Azmitia 1992), all of which are r5 anatomic markers. In our material, the $\mathrm{RMg}$ population seems to be larger and more compact within r6 (because of less decussating fibers?). In fact, the RMg topography proposed by Steinbusch and Nieuwenhuys (1983) restricts it completely to r6, since these authors hold that it is coextensive with the facial motor nucleus. We agree with these authors that the raphe cells found in $\mathrm{r} 7$, intercalated between the facial nucleus and the superior olive, are best assigned to the $\mathrm{RPa} / \mathrm{ROb}$ complex. We conclude that this classic nucleus in any case occupies a retropontine position within the pontomedullary region, and we tentatively define its rhombomeric extent as occupying r5 and r6.

Similar calculations were done for placing the other caudal raphe formations, since available descriptions were rather variable. The rostral end of the $\mathrm{ROb}$ was described by Jacobs and Azmitia (1992) as at level with the VI nucleus or VI nerve root (r5), while the RPa was reported by other authors (Olszewski and Baxter 1954; Taber et al. 1960) to stop rostrally at the level of the middle of the motor facial nucleus (r6) or, alternatively, at the rostral pole of the inferior olive (r8; Hornung and Fritschy 1988; Jacobs and Azmitia 1992). These differences in description probably obey to variations in the sectioning plane. We think that it is not evident from the literature that $\mathrm{ROb}$ and $\mathrm{RPa}$ coincide in reasonable cross sections with $\mathrm{RMg}$, though they do coincide with each other. This is particularly clear when sagittal sections are studied (Fig. 3). We therefore suggest that $\mathrm{ROb}$ and RPa both begin rostrally in $\mathrm{r} 7$, once $\mathrm{RMg}$ ends in $\mathrm{r} 6$. The $\mathrm{r} 6 / \mathrm{r} 7$ boundary happens to correlate with the change from overt rhombomeres to cryptorhombomeres (Cambronero and Puelles 2000; Watson et al. 2010), as well as with the transition of the hindbrain pontine and retropontine molecular domains controlled by the Hoxl-Hox3 gene paralogs into the domains controlled by the Hox4-Hox7 paralogs (Marín et al. 2008); the r6/r7 boundary therefore may explain the $\mathrm{RMg}$ versus $\mathrm{ROb} / \mathrm{RPa}$ structural and typological transition. The ROb and RPa complexes emerge accordingly as being coextensive with the cryptorhombomeres r7-r11. Since these developmental units tend to develop very similar structures (metamery, or plurineuromeric regularity), causing the appearance of apparently continuous columnar plurineuromeric complexes, this would explain that both $\mathrm{ROb}$ and $\mathrm{RPa}$ have been always interpreted as single entities. An increase in shared morphological characteristics is noted throughout in these caudal hindbrain developmental units (predominant columnar structure of all nuclei in the caudal medulla).

Apart of the r5 and r6 portions, we propose a dorsoventral subdivision of the $\mathrm{RMg}$ complex into a slightly dispersed intermediate stratum component (RMgD), and a more compact ventral or superficial part $(\mathrm{RMgV})$; this notion was already introduced by Puelles et al. (2007) in the chick atlas. There is evidence that the $\mathrm{RMgD}$ and $\mathrm{RMgV}$ cell populations have different patterns of differentiation, so that RMgV develops first (Lidov and Molliver 1982; Wallace and Lauder 1983). Interestingly, there is also some evidence of neuronal typological differences within the retropontine $\mathrm{RMg}$ complex, which may correlate with the alternative r5 versus r6 topographies (see Hornung and Fritschy 1988). As regards chemoarchitectonic properties, whereas SP neurons are detected in both $\mathrm{r} 5$ and $\mathrm{r} 6$ parts of $\mathrm{RMgD}$, $\mathrm{TH}$ neurons are detected at the $\mathrm{r} 5 \mathrm{RMgV}$, but are absent at r6RMgV (Allen Developing Mouse Brain Atlas; http://www.developingmouse.brain-maps.org; Halliday et al. 1988; Rikard-Bell et al. 1990; Poulat et al. 1992; Wu et al. 1993; Hornung 2003). In any case, TH neurons probably are migrated from non-raphe sources, and would not be very significant.

Differential segmental patterns are therefore less obvious in the cryptorhombomeric raphe nuclei ( $\mathrm{ROb}, \mathrm{RPa}$; Steinbusch and Nieuwenhuys 1983; Hornung and Fritschy 1988; Jacobs and Azmitia 1992; Bjarkam et al. 1997; Cambronero and Puelles 2000; Nieuwenhuys et al. 2008). They belong to a hindbrain subregion that is devoid of overt rhombomeric limits, and adjacent developmental units tend to be homogeneous in their histogenesis, irrespective of the local differential patterns of expression of Hox gene paralog groups (see Marín et al. 2008). Raphe nuclei of $\mathrm{r} 7$ and $\mathrm{r} 8$ seem to lack cells expressing SP or TH, unlike raphe nuclei in $\mathrm{r} 5$ and $\mathrm{r} 6$; such cells reappear in the r9 and r10 ROb units (Del Fiacco et al. 1984; Halliday et al. 1988; Rikard-Bell et al. 1990).

Lateral or parapyramidal serotonergic cells of the caudal cluster appear early in development, as observed at the rostral cluster. They extend longitudinally between $\mathrm{r} 5$ and r11 (Lidov and Molliver 1982; Wallace and Lauder 1983; 
Aitken and Törk 1988; present results). The serotonergic parapyramidal cells within $\mathrm{r} 5$ and $\mathrm{r} 6$ are conventionally described as located in the 'lateral paragigantocellular nucleus' (LPGi) (Jacobs and Azmitia 1992), or in the 'rostral ventrolateral medulla' (Törk 1990; Harding et al. 2004). Their lateral position results from a migration pattern that resembles the one postulated for the rostral SuL cluster (Hawthorne et al. 2010), but associated in this case to the source of the RMg cells in r5 and r6. We feel it may be clarifying to refer to these cells as $r 5$ and r6 parts of the PPy column, to emphasize the regional similarity with the more caudal medullary PPy group.

The medullary PPy was identified within r7-r11, surrounding laterally the inferior olive (once the inferior olive ends, these cells surround the pyramidal tracts, or intermix with the pyramidal decussation). These cells were previously vaguely included in the RPa nuclear complex, or their position was defined as occupying the 'caudal ventrolateral medulla' (Törk 1990; Harding et al. 2004). We thus propose to classify them as a continuous series of rhombomeric PPy raphe cell groups (r7PPy-r11PPy).

A peculiarity of the caudal cluster is the general absence of periventricular serotonergic populations. This pattern is permanent in the cryptorhombomeres r7-r11, but some small 5-HT-immunoreactive periventricular neurons appeared in our postnatal material, associated to the supragenual area in the pontomedullary rhombomeres $r 5$ and r6. They correspond to the 'extraraphe' cells described by Olszewski and Baxter (1954), or the B4 cell group mentioned by Dahlström and Fuxe (1964). A retarded postnatal neurogenesis and differentiation of these paramedian cells does not seem plausible, since hindbrain neurogenesis is held to stop long before; late birthdates might be compatible with a source in the r5-r6 rhombic lip. It will be necessary to investigate a possible tangential migration of these cells into this position; the possibility that these cells are non-neuronal has to be investigated as well, since we did not detect any expression of Petl at this place at either embryonic or postnatal stages. Indeed, some hypothalamic tanycytes were found to be immunoreactive for serotonin (Steinbusch and Nieuwenhuys 1983) due to transmembrane transport of monoamines from the surrounding medium (Ugrumov et al. 1989; Ugrumov 1997; Hansson et al. 1998).

The raphe pontis nucleus in $\mathrm{r} 4$

Our results demonstrate the presence of serotonergic neurons in $\mathrm{r} 4$ in mouse brains at embryonic and postnatal stages (our r4PnR cell group). However, the absence of serotonergic neurons in $\mathrm{r} 4$ is an accepted dogma in the field (e.g., Jensen et al. 2008). Studies focused on the molecular profile of this rhombomere at early stages demonstrated a sustained production of branchiomotor neurons (bMNs) and postulated, as a consequence, a local inhibition of serotonergic neuron production (Pattyn et al. 2003; Jacob et al. 2007). Such inhibition is attributed to collateral effects of the transcription factor Phox $2 b$, maintained locally by Hoxbl, on the production of bMNs (Pattyn et al. 2003). These studies were restricted to relatively early stages (E9.5-E11.5), when bMNs of the facial motor nucleus are generated (Goddard et al. 1996; Studer et al. 1996). Differentiation of serotonergic neurons apparently was not explored at later stages, which is when we detected such neurons in $\mathrm{r} 4$ (from E12.5 onwards). Thus, our results indicate instead a heterochronic biphasic pattern in $\mathrm{r} 4$, first with prolonged local generation of bMNs, followed by delayed production of a few serotonergic neurons, possibly bespeaking of a skewed probabilistic control mechanism of the fate choice done by the relevant postmitotic neurons.

We observed that $\mathrm{r} 4$ pontine raphe neurons are not transient, since they persist at postnatal stages (present results) and in adults (data not shown). Also, they are not a peculiarity of the mouse brain, since it is possible to confirm their existence in other mammals, though the r4RPn nucleus tends to be classified as a rostral component of RMg (Taber et al. 1960; Skagerberg and Björklund 1985; Hornung and Fritschy 1988). Similar pontine r4 raphe cells were found as well in sauropsides (chick; Cambronero 1999; reptiles; Rodrigues et al. 2008; their Fig. 1).

In addition to a delayed and diminished production of serotonergic cells, there exists also a peculiar transcriptional regulation of the serotonergic phenotype in $\mathrm{r} 4$, at least in the mouse. The r4PnR neurons are positive for Gata3, Lmxlb and Petl, but we did not detect the expression of Gata2, Tph2 and Slc6a4 in this group. The absence of Gata2 signal suggests that this gene is not necessary to determine the serotonergic phenotype in $\mathrm{r} 4$, this role probably being assumed solely by Gata3, similarly as occurs in the caudal cluster (van Doorninck et al. 1999; see below). The most striking molecular deviation of this serotonergic group is the very low or absent signal of Tph2, despite the normal expression of Pet1 and $L m x 1 b$, and the presence of immunoreactive 5HT. A plausible interpretation predicts the existence of a particular isoform of tryptophan hydroxylase (TPH) in r4, which is not detected by our Tph2 riboprobe. An alternative explanation is that these neurons are unable to synthesize TPH enzymes, due to the absence of some transcription factor necessary to regulate the expression of its messenger (for example Gata2), but they have the capacity to synthesize the serotonin transporter (Slc6a4) - under independent regulation by Pet1 and Lmx $1 b$ - and, therefore, can take up serotonin present in their environment. Although we did not detect ourselves Slc6a4 expression during mouse development (negative data attributed to malfunctioning of the probe used), 
positive data of its expression at E15.5-P14 are found in the Allen Developing Mouse Brain Atlas (http://www. developingmouse.brain-map.org), supporting the latter hypothesis. Neurons that take up serotonin, but do not synthesize it, are known in the hypothalamus (Ugrumov et al. 1989; Ugrumov 1997; Hansson et al. 1998). The status of these r4RPn neurons as bona fide serotonergic neurons (Hoffman et al. 1998) is therefore still controversial, until the doubt about TPH is resolved.

\section{Serotonergic populations outside the hindbrain}

We detected only two serotonergic populations lying outside the rhombomeric territory. These were placed, respectively, at the rostral and caudal ends of the hindbrain raphe system: the midbrain mDR cell group and a small serotonergic group in the cervical spinal cord. Immunoreactive serotonergic neurons start to appear in the caudal midbrain at E14.5 (though some Petl-expressing cells were found at E12.5), and form the sizeable mDR group just rostral to the isDR. The $\mathrm{mDR}$, which corresponds to what some authors identify as 'rostral DR nucleus' (Hale and Lowry 2011), is restricted to the small preisthmic region (recently redefined as mesomere 2-m2-of the midbrain; Hidalgo-Sánchez et al. 2005; Martínez et al. 2012; Puelles et al. 2012b; Allen Developing Mouse Brain Atlas, http://www.developingmouse.brain-map.org). In contrast to conventional attribution of the rostral raphe cluster as a whole to either the midbrain, or the hindbrain (Jensen et al. 2008), these are serotonergic neurons that are truly present in the adult midbrain, as long as the caudal end of the Otx2 expression domain is accepted as the midbrain-hindbrain boundary (Puelles et al. 2012b). The genetic profile of the mDR resembles that of its neighbor, the isDR, suggesting that these neurons may migrate tangentially from the isthmus, where they would be born. Alternatively, the parallel characteristics may be due to similar effects produced by the isthmic organizer on both $\mathrm{m} 2$ and isthmic progenitors. The migration hypothesis is supported by their early absence at the midbrain at E12.5, and their gradual appearance at E14.5, shaped as a rostrally pointing spike connected with the isDR across the molecular Otx2-labeled MHB. This boundary is known to be permissive to tangential neuronal migration between midbrain and hindbrain in both senses. Kala et al. (2008) studied in transgenic mice the distribution of MHB-Cre labeled neurons derived from rostral ' $\mathrm{r} 1$ ' (meaning essentially the isthmic region), and found evidence of labeled cells entering the caudal midbrain (what we interpret as $\mathrm{m} 2$ ), presumably representing or including the mDR cells. Similarly, Zervas et al. (2004) studied the Wntl-related lineage (Wnt1-CreER), supposedly restricted to the midbrain, finding that the labeled cells are majoritarily dopaminergic, but that they intercalate with some unlabeled serotonergic neurons (their Fig. 3c); these would have migrated from the isthmus; however, they also found some isolated double-labeled cells. Puelles et al. (2004) suppressed Otx2 expression in the basal plate of the midbrain, implicitly modifying the local molecular identity, or the site of the functional MHB. This caused an expansion of $N k x 2.2$ expression in the midbrain basal plate, and a consequent reduction of dopaminergic neurons in favor of serotonergic ones (the authors did not determine whether the latter were generated locally, or migrated from the isthmus). Curiously, we observed that as the mDR starts to form, Otx2 diminishes or disappears at its location (see our Fig. 4d-f). It is unclear whether down-regulation of this gene precedes (and maybe causes) the appearance/ migration of the $\mathrm{mDR}$, or is rather a consequence of its migratory formation. In any case, the phenomenon bespeaks of a possible cross-repressive molecular interaction between $\mathrm{m} 2$ and isthmic derivatives. On the other hand, apart of isthmic serotonergic neurons that seem to invade the midbrain, there exist also midbrain dopaminergic neurons that seem to invade secondarily the isthmic tegmentum (LP, unpublished observations). Finally, it is interesting to note that the observations of Sako et al. (1986) and Cambronero (1999) on DR serotonergic neurons in the chick did not disclose any significant $\mathrm{mDR}$ homolog, since only few isolated elements appeared transiently rostral to the MHB.

Similarly as other authors (e.g., Törk and Hornung 1990; Jacobs and Azmitia 1992), we found 5HT-positive neurons in the upper cervical levels of the spinal cord. These neurons appear approximately at the same time as their rostral neighbors in the RPa and ROb nuclei (at E12.5, according to our results). These data suggest an in situ origin, rather than a migration, though we cannot discard that possibility. Their low number might be an effect of repressing retinoic acid signals from the caudal secondary organizer (Díez del Corral and Storey 2004).

Segmental organization of raphe nuclei correlated with their developmental genoarchitecture

The raphe nuclei are distributed rostrocaudally throughout the rhombencephalic paramedian basal plate, with minimal invasion of midbrain and spinal cord. This represents accordingly a shared histogenetic feature of the whole set of rhombomeres constituting the hindbrain tagma (isthmus, r1-r11). This feature probably can be attributed to a comparable influence of notochordal and floorplate-derived Shh signals on this part of the neural tube, with immediate effects on both the serotonergic and motoneuronal populations (Briscoe et al. 1999). Further effects downstream of Shh, involving $N k x 2.2, L m x 1 b$ and the Gata 2/3 genes in the absence of Otx2 apparently lead to the serotonin 
transmitter phenotype, with associated differentiation markers. Notwithstanding this common causal scenario repeated along the hindbrain AP axis, there are overlapping, variously nested expression patterns of Hox homeodomain genes and other hindbrain differential molecular determinants, which correlate causally with hindbrain segmentation (Lumsden and Krumlauf 1996; Marín et al. 2008). The resulting differential genoarchitectonic profiles of the rhombomeres provide regional differences in genomic regulation, which allow in principle individual raphe nuclei to become distinct one from another, as occurs with other derivatives of these developmental units (motor and sensory populations, reticular cells, etc.). Our present approach included examining whether given molecular features differ between the diverse raphe populations, presumably as a result of their segmental identity and particular histogenetic conditions (e.g., radial or lateral migration).

Segmental identity in terms of a specific set of active genes is imprinted early on the neuroepithelial progenitor cells, generally shortly before neurogenesis begins, and such identity may be inherited or diversified subsequently in particular neuronal derivatives, as emergent genoarchitecture (Ferrán et al. 2009). Serotonergic neurons share common progenitors with visceromotor ( $\mathrm{vMN})$ and branchiomotor (bMN) neurons (Briscoe et al. 1999; Pattyn et al. 2003; Jacob et al. 2007), though there are exceptions; there are no vMNs and bMNs at the Is and $\mathrm{r} 1$, and $\mathrm{r} 4$ is supposed to lack serotonergic neurons, or produces few of them (Weilan et al. 1998; Briscoe et al. 1999; Ding et al. 2003; Pattyn et al. 2003; Jacob et al. 2007). Didactic generalization to the whole hindbrain of results obtained in individual rhombomeres can omit segmental particularities worthy of consideration. In the end, developmental programs must exist in each rhombomere that enable production of specific visceromotor and/or branchiomotor neurons, plus specific serotonergic neurons, among other specific anatomical derivatives. Our results on genes expressed in postmitotic serotonergic neurons show that there exist indeed peculiarities related to rhombomeric topography, as well as some variations occurring during development (Fig. 13; see also Wylie et al. 2010).

Among the genes examined, Enl and En2 are expressed at the midbrain and rostral hindbrain, down to $\mathrm{r} 1$, and thus their signaling has a restricted position with regard to the whole set of raphe nuclei (Wylie et al. 2010; Fox and Deneris 2012; present results). Data from En mutants (Simon et al. 2005) indicate there is no phenotype caudal to r1. The DR, CLi and MnR nuclei are lost, but not so PPnR (in $\mathrm{r} 2$ ) and $\mathrm{r} 3 \mathrm{PnR}$ (for clarity, we interpret the reported data according to our terminology). In our material, En2 signal is first restricted (up to E16.5), in a gradient decreasing caudalwards, to the ventricular zone of Is-r1r, but is lost by
E18.5, suggesting a transient role in the differential specification of the local serotonergic derivatives (isDR, r1DR, $\mathrm{CLi}$ and MnR). It is tempting to speculate that the En2positive domain, representing the range of Fgf8 morphogen signaling from the isthmic organizer, may embody a local molecular context that prohibits local differentiation of bMNs (the rostralmost hindbrain bMNs pertain to $\mathrm{r} 2$ ), and only allows MNs at a restricted rostral locus in the isthmus (trochlear nucleus). The domain of Enl expression essentially overlaps spatially and in its gradiental aspect that of En2, but expression extends also to the periventricular and intermediate strata of serotonergic cells in the mantle. This pattern persists at least until postnatal stages, suggesting a supportive role in the maintenance of some aspect of the local serotonergic cells (personal observations, Allen Developing Mouse Brain Atlas, http://developingmouse.brain-map.org/). A minor variant, which can be attributed to migratory displacement, is the presence of a patch of Enl-positive neurons in the PPnR (in r2; Jensen et al., 2008). It has been postulated that En1 is involved in the maintenance of the serotonergic phenotype in the hindbrain, similar to its role with regard to the dopaminergic phenotype in the midbrain (Simon et al. 2005). This phenotypic duality seems related to differential fate regulation due to overlapping expression of Otx2 in the midbrain (Brodski et al. 2003; Puelles et al. 2004; Simeone et al. 2011).

The other six genes studied by us (Gata2, Gata3, Lmxlb, Pet1, Tph2 and Slc6a4) are expressed in most serotonergic groups, implying a fundamental relationship with the neurotransmitter phenotype (Deneris and Wyler 2012), rather than with differential segmental identity. Expression of Gata2 precedes that of Gata3 (Nardelli et al. 1999). As happens in hematopoietic cells (Ferreira et al. 2007), variations in the regional expression levels of the Gata genes may relate to different dosage requirements in distinct serotonergic nuclei (we observed highest levels of transcription in the lateral raphe nuclei of $\mathrm{r} 5$ and $\mathrm{r} 6$ ). Interestingly, the functional roles of these two genes differ as regards the rostral and caudal raphe clusters, since the former selectively requires Gata2, whereas the latter, and specially the ROb nuclear complex, needs Gata3 (Nardelli et al. 1999; van Doorninck et al. 1999; Craven et al. 2004). These data reveal a particular genetic regulation requirement for the cryptorhombomeric serotonergic groups, or at least for some particular raphe populations within them. In any case, the onset of Gata3 expression correlates with that of Lmxlb and Pet1.

$L m x 1 b$ and Petl appear to play important roles in the differentiation and maintenance of the serotonin phenotype, though the ampler initial expression domain of $L m x I b$ in the hindbrain-notably in the periventricular stratum throughout the length of the hindbrain-suggests that it 
controls as well other neuronal phenotypes. As development advances, these non-serotonergic extra populations gradually lose the Lmxlb signal. Note that $L m x l b$ is expressed likewise initially along the midbrain, diencephalic and hypothalamic basal and floor plates, in connection with the production of dopaminergic neurons and probably also other local cell types. Lmxlb apparently acts upstream of Pet1 in hindbrain serotonergic neurons (Hendricks et al. 1999; Pfaar et al. 2002). Although the loss-offunction phenotypes of both genes are similar (Hendricks et al. 2003; Ding et al. 2003), we observed differences in their respective hindbrain expression patterns. In the rostral cluster, Lmxlb signal appears in a rostro-caudal gradient (Ding et al. 2003; present results), not observed with Pet1; this gradient is consistent with the En1/En2 gradients and the spatial gradient of differentiation of 5HT-immunoreactive neurons. This pattern is maintained into adulthood, probably due to a maintenance function of the serotonergic lineage similar to that observed for the dopaminergic lineage (Smidt et al. 2000), which would seem to require highest dosage rostrally. At E14.5 and E18.5 we observed distinctly stronger expression of $L m x l b$ at the MnRr than at MnRc.

In the caudal cluster, Lmxlb signal has a rostrocaudal gradiental expression pattern which roughly agrees again with the spatial pattern of maturation of 5HT neurons; these differentiate first in r6 (normally the paired rhombomeres are advanced in neurogenesis relative to unpaired ones), extending immediately afterwards to $\mathrm{r} 5$ (Cambronero 1999) and gradually into the r7-r11 series (Wallace and Lauder 1983; Pattyn et al. 2003). The medullary groups may have a lower requirement of $L m x l b$ for their differentiation, if other transcription factors, such as Gata3 or Pet1 play a complementary role. We observed that the $L m x l b$ signal is patchy and is completely switched off at the caudal cryptorhombomeres at late developmental stages; this suggests that this gene does not have a relevant function in serotonergic lineage maintenance in the medulla. Pet 1 might replace $L m x l b$ in this regard, since its expression is more homogeneous, and, moreover, it is known to be required for the maintenance of the serotonergic phenotype in adults (Liu et al. 2010; Song et al. 2011).

At early stages, Petl signal presents some peculiarities relative to segmental raphe populations, since it first appears in a non-gradiental, irregular pattern (Fig. 13). These differences apparently reflect heterochronic rhombomere-specific regulatory programs for the expression of this gene. Consistently with this idea, the Petl-null mutant conserves selectively Pet1-positive populations at the isthmus, probably due to the agency of a separate enhancer (Hendricks et al. 2003; their Fig. 2). This serotonergic population roughly coincides with the $T p h 2$ positive neurons that are conserved in Tph2-conditional mutants
(Kriegebaum et al. 2010), suggesting a close relationship in the regulation of $T p h 2$ expression by Pet1 at isthmic levels. On the other hand, Petl and $L m x l b$ are jointly implicated in the regulation of Slc6a4 throughout the set of raphe primordia (Hendricks et al. 1999, 2003; Zhao et al. 2006), but the expression of the latter is delayed compared with that of $T p h 2$. This marker also shows various heterochronic aspects among specific segmental serotonergic groups (Fig. 13).

We searched for genes displaying an expression pattern restricted to some raphe nuclei in the Allen Mouse Brain Atlas database (http://mouse.brain-map.org). We found genes expressed in some cell aggregates or scattered cells in both clusters, and other genes expressed only in some rostral or caudal cluster subdivisions. We noted that such genes are not limited to those involved in the specification or maintenance of the serotonergic phenotype. Some of the genes identified-Cbln2, Grm3, Chrna7, and Trh-are probably involved in the modulation of serotonergic functions. Cbln2 is implicated in the formation of a kind of excitatory synapse, and in synaptic communication, in the central nervous system (Eiberger and Schilling 2012). In serotonergic subpopulations, it is possibly related to glutamatergic modulation of this neuronal phenotype (Soiza-Reilly and Commons 2011), as occurs likewise with Grm3 (Harrison et al. 2008). The function of Chrna7 is less clear, but the serotonergic and cholinergic systems apparently modulate themselves mutually in some cognitive functions such as learning and memory (Garcia-Alloza et al. 2006); the regionally restricted expression of this gene within the raphe system suggests that such modulation may be particularly relevant in the serotonergic subpopulations where it is expressed. Trh is a hormone implicated in the modulation of arousal, cognition, motor functions and pain (Boschi et al. 1983; Nillni and Sevarino 1999), in addition to its endocrine actions. Curiously, Trh is expressed selectively at the $\mathrm{RMgV}$ (the 'rostral ventromedial medulla' of Porreca et al. 2002; see our Fig. 14c), which is involved in modulation of ascending pain signal transmission, thanks to its descending projections to the dorsal horn of the spinal cord.

Other genes with restricted expression within the raphe populations remain devoid of a clear role where they appear expressed. Cart induces neurite elongation and ramification in dopaminergic, hippocampal, retinal and motoneurons primary cellular cultures (Rodrigues et al. 2011); maybe it is related with some local aspects of preand postnatal development of serotonergic subpopulations, including synaptogenesis. Hdac6 reportedly has a role in glucocorticoid-receptor-related homeostasis of particular raphe circuits related to social behavior, particularly in the DR nucleus (Espallergues et al. 2012). No known function can be attributed to the transcription factors $Z f h x l b$ and 
Lhx3, and the orphan nuclear receptor Esrrb, in the serotonergic subpopulations and neighboring cells in which they are expressed.

Do segmental components of the raphe nuclei have heterogeneous connectivity patterns?

The diverse connectivity patterns so far described for the raphe nuclei probably are best explained by their segmental organization. A recent publication of Bang et al. (2012) indeed strongly supports that idea, even though the flatmap graphic representation of their results in their Fig. 5 is surprising in lacking transverse rhombomeres at all, and inexplicably represents median or paramedian raphe or dopaminergic cell populations (VTA, MR, DR) at a considerable distance from the flatmap midline. Molliver (1987) referred to serotonin projections as evidencing "multiple neuronal subsystems that have high degree of specificity and precision in their organization". The modular character of the rhombomeric derivatives allows in principle the emergence of these characteristics, since individual subpopulations may develop quite diverse sets of efferent and afferent connections, while conserving some shared features (e.g., joint axonal navigation rules with opportunistic properties). A segmental connectivity pattern is clearest at the rostral cluster nuclei, which also have received more attention in hodologic studies.

There exists some degree of segmental hodologic specialization of the isthmic DR subnuclei-isDR jointly with the $\mathrm{mDR}$ - versus those derived from $\mathrm{r} 1$. These patterns were lumped by Bang et al. (2012) because of their joint labeling of midbrain, isthmic and $\mathrm{r} 1$ raphe component projections derived from their composite ' $\mathrm{rl}$ ' domain. The $\mathrm{mDR} / \mathrm{isDR}$ neurons apparently preferentially project upon centers involved in motor control—substantia nigra, caudate/putamen - while the r1DR neurons project to limbic system formations-hippocampus, locus coeruleus-(Fuxe et al. 1977; Köhler and Steinbusch 1982; O'Hearn and Molliver 1984; Imai et al. 1986; Mamounas et al. 1991; Vertes 1991; Jacobs and Azmitia 1992; Waselus et al. 2006). Some segmental specialization of efferences apparently also occurs in other populations-MnR, PPnR, $\mathrm{PnR}, \mathrm{RMg}$, and medullary raphe nuclei-although the available data are scarce and often contradictory.

An example that illustrates this is the attribution of a variety of efferent targets to the $\mathrm{MnR}$ in the literature, probably due to the fact that many authors lump in this complex different sets of raphe neurons actually located in r1, r2, or even r3 (Törk and Hornung 1990; Vertes et al. 1999). According to earlier, less clearcut evidence, the $\mathrm{MnRr}$ apparently sends axons to the amygdala, hippocampus, septum, diagonal band and (probably) the rostral IP, while the MnRc projects specifically to the hypothalamus (some authors emphasize the suprachiasmatic nucleus), DR, ventral tegmental area, substantia nigra pars compacta, and (probably) the caudal IP nucleus (Imai et al. 1986; Vertes and Martin 1988; Meyer-Bernstein and Morin 1996; Vertes et al. 1999). Taking into consideration the relevant selective labeling of $\mathrm{r} 2$ raphe projections reported by Bang et al. (2012), it would seem that the cells innervating selectively the suprachiasmatic nucleus derive from $\mathrm{r} 2$, even if they occupy a place in MnRc, which lies in caudal r1. Such movements to neighboring raphe domains are by no means impossible (Jensen et al. 2008). Another selective projection of $\mathrm{r} 2$ raphe cells (it is unclear whether we deal here with PPnR) is to the posterior periventricular nucleus of the thalamus (Bang et al. 2012). It is otherwise very difficult to dissociate among all available data those projections that may concern specifically the PPnR ( $\mathrm{r} 2)$, in contrast to the MnRc. It is therefore highly plausible that some of the heterogeneous connections attributed to the MnRc actually belong to the PPnR, or to cells migrated from $\mathrm{r} 2$ into $\mathrm{r} 1$.

Few hodological studies mention specific connections attributed to the PnR (r3PnR in our interpretation), though some results suggest that group projects mainly to visuomotor centers, such as preoculomotor reticular neurons, the superior colliculus and some pretectal nuclei, in addition to the cerebellar vermis (Bobillier et al. 1976; Päällysaho et al. 1991). However, none of these targets was mentioned in the recent description of $\mathrm{r} 3+\mathrm{r} 5$ raphe projections (Bang et al. 2012); these authors emphasized instead forebrain projections largely shared with ' $\mathrm{r} 1$ ' and $\mathrm{r} 2$ raphe neurons, with subtle differences (mainly at the neocortex and amygdala), and connections directed toward other raphe nuclei (MnR, DR), the lateral parabrachial nucleus, the locus coeruleus, the anterior or ventral tegmental nucleus (a target we would interpret rather as the rhabdoid nucleus, due to its characteristic size, shape and paramedian position behind the decussation of the brachium conjunctivum) and the dorsal tegmental nucleus.

The supralemniscal raphe nuclei extending across $\mathrm{r} 1-\mathrm{r} 3$ also show some differences in connectivity, once the data available are poured into our interpretive schema. The study of Vertes and Martin (1988) reported efferents to the central IP subnuclei, retrorubral area, substantia nigra pars reticulata, anterior pretectal nucleus, the thalamic anterior intralaminar complex, the suprachiasmatic nucleus and some other hypothalamic centers, such as the retromammillary nucleus and the mammillary body, and the preoptic area, which we believe (according to the retrograde mappings themselves) map selectively to the r1SuL, which the authors identified as the 'nucleus pontis oralis'. In contrast, we interpret retrograde hodological mappings of reported projections to the MnR (Stratford and Wirtshafter 1988), the prethalamic reticular nucleus (Rodríguez 
et al. 2011), the arcuate and ventromedial hypothalamic nuclei (Willoughby and Blessing 1987) and the entorhinal cortex (Köhler and Steinbusch 1982) as labeling rather selectively the $\mathrm{r} 2 \mathrm{SuL}$ and/or r3SuL cell populations.

In the case of the caudal raphe groups, some confusion results from the circumstance that their descending axons send collaterals to diverse spinal cord segments; the shared axonal navigational properties have probably blurred any specific segmental origins of given connectivity patterns (Harding et al. 2004). Even so, it was shown that r5RMg neurons project specifically to the dorsal horn of the spinal cord (Ruda et al. 1982; Hylden et al. 1986; Jacobs and Azmitia 1992), whereas r6RMg neurons project instead to periventricular layer $\mathrm{X}$ of the cervical spinal cord and to the spinal trigeminal nucleus (Beitz 1982; Azmitia and Gannon 1986; Jacobs and Azmitia 1992). There are also striking differences between the efferents of the r5 and r6 parts of the PPy formation. The r5PPy neurons project to the intermediate sensorimotor zone of the spinal cord, where the autonomic sympathetic preganglionic neurons are found (Bowker et al. 1982), while the r6PPy neurons establish connections with the brainstem ventral respiratory groups (Ellenberger and Feldman 1990; Morillo et al. 1995), the spinal dorsal and ventral horns, and the sacral parasympathetic preganglionic population (Hermann et al. 2003).

As regards the medullary raphe groups, the paramedian elements generally project to motor neurons of the brainstem (ROb; Felten and Sladek 1983) and the ventral horn of the spinal cord ( $\mathrm{ROb}$ and $\mathrm{RPa}$; Azmitia and Gannon 1986; Sasek et al. 1990; Veasey et al. 1995). In its turn, the medullary parapyramidal nuclei (r7-r11PPy) connect generally with the preganglionic neurons of the autonomic nervous system (Sasek et al. 1990). In these cases, potential rhombomeric specificities have not been explored, and do not transcend from published material.

Within each rhombomere, the radial and medio-lateral subdivisions of the local serotonergic nuclei apparently may have differential efferent targets (Imai et al. 1986; Vertes et al. 1999). The clearest data supporting such specialization belong to the isDR complex, since its medial subdivisions (isDRd, isDRv) send ascending projections to somatosensory centers-somatosensory thalamus (trigeminal) and cortex-(Kirifides et al. 2001; Lee et al. 2008), while the lateral subdivision (isDRW) projects instead to visual centers (Pasquier and Villar 1982; Villar et al. 1988; Waterhouse et al. 1993). More detailed genoarchitectonic studies of raphe subdivisions and their development are needed to examine the causes of such connective specialization.

Bang et al. (2012) have further underlined the existence of selective innervation of some serotonergic raphe cells by collaterals coming from specific rhombomeric populations (for instance, DR axons project into $\mathrm{MnR}, \mathrm{RMg}$ and $\mathrm{ROb}$, r2-derived serotonergic terminals surround non-r2 MnR neurons, and $\mathrm{r} 3+\mathrm{r} 5$ originated raphe axons selectively reach the DR). These synapses are held to generate collateral modulatory inhibitory effects via the $5 \mathrm{HT} 1 \mathrm{~A}$ receptor.

The general conclusion from this analysis is that all connections of the raphe nuclei need to be examined further taking in consideration their rhombomeric position and boundaries. The same applies to afferences to these nuclei. Some mutant mouse lines are presently available (and more will accrue) that are useful to test the role of specific rhombomeres in the development and function of given connections. Such an effort should enhance significantly our understanding of the functions of serotonergic signaling in general, probably discriminating a number of discernible subsystems, and throwing light on various pathophysiological aspects.

\section{Conclusions}

The serotonergic phenotype is associated to a single paramedian progenitor domain along the hindbrain tagma, which is subdivided into 12 segmental portions (isthmus, r1-r11; Fig. 15c), and seems complemented by neighboring minor domains in the caudal midbrain and rostral spinal cord (it remains unclear whether intrinsic patterning or tangential migrations are involved in these additions). We also found that $\mathrm{r} 4$ produces a small number of serotonergic neurons, instead of being a gap rhombomere, unable to produce serotonergic neurons, as is usually thought. There is evidence that, irrespective of the common neurotransmitter phenotype, the cells produced at each segmental level variously reflect the local molecular context (rhombomeric identity) in terms of specific gene expression patterns or gradiental expression patterns, varying layering behaviors (superficial, intermediate or periventricular sites), lateral dispersion behaviors, cell typology, and specific projection patterns. Individual rhombomeres may reproduce or not the overall pattern found in their immediate neighbors, sometimes forming plurineuromeric complexes. Under this light, the classic raphe nuclei can be understood as plurineuromeric complexes, whose cytoarchitectonic definition resulted from lumping together cell groups showing a similar histogenetic pattern consecutively in a few adjacent rhombomeres (for instance DR across $\mathrm{m} 2$, Is and $\mathrm{r} 1, \mathrm{RMg}$, across $\mathrm{r} 5$ and $\mathrm{r} 6$, or RPa and $\mathrm{ROb}$ across $\mathrm{r} 7-\mathrm{r} 11)$. This raises the issue whether individual rhombomeric components of such complex anatomic units have shared connectivity and functional properties, or display segmental differences due to local molecular singularities. The literature already contains some data suggesting that the latter case is true, but more research done with this possibility in mind is needed. In 
this sense, our updated terminology, which adds a segmental reference code for the individual parts, should help in producing more precise descriptions of observed hodologic differences and other differential properties. A similar analysis obviously applies to other hindbrain 'columnar' nuclei (Marín and Puelles 1995; Cambronero and Puelles 2000; Marín et al. 2008).

Acknowledgments Grant sponsor: Spanish Ministry of Education and Science; Grant number: BFU2008-04156; Grant sponsor: SENECA Foundation; Grant number: 04548/GERM/06 (no. 10891); Grant sponsor: Centro de Investigaciones Biomédicas en Red de Enfermedades Raras, Instituto Carlos III, Madrid, Spain (CIBER-ER; intramural project between units U736, L.P., and U703, R.A.); J.L.F. and A.A. were postdoctoral researchers at the CIBER-ER; Grant sponsor: National Institute of Health, Bethesda, USA; Grant number: 1-R01-MH070370-01A2 (postdoctoral fellowship to P.M. and predoctoral fellowships to J.E.S.); Grant sponsor: Spanish Ministry of Science; Grant number: BFU2008-04156; FPI predoctoral fellowship to L.S-A. We thank Isabel Piqueras and Tania López for expert technical assistance. We also thank Faustino Marín and Pilar Aroca for helpful discussion of results and criticism of the manuscript.

Conflict of interest The authors state that no conflict of interest is involved in the present publication.

Open Access This article is distributed under the terms of the Creative Commons Attribution License which permits any use, distribution, and reproduction in any medium, provided the original author(s) and the source are credited.

\section{References}

Abrams JK, Johnson PL, Hollis JH, Lowry CA (2004) Anatomic and functional topography of the dorsal raphe nucleus. Ann N Y Acad Sci 1018:46-57

Agnati LF, Fuxe K, Hökfelt T, Benfenati F, Calza L, Johansson O, De Mey J (1982) Morphometric characterization of transmitteridentified nerve cell groups: analysis of mesencephalic 5-HT nerve cell bodies. Brain Res Bull 9:45-51

Aitken AR, Törk I (1988) Early development of serotonin-containing neurons and pathways as seen in wholemount preparations of the fetal rat brain. J Comp Neurol 274:32-47

Araneda S, Gysling K, Calas A (1999) Raphe serotonergic neurons projecting to the olfactory bulb contain galanin or somatostatin, but not neurotensin. Brain Res Bull 49:209-214

Aroca P, Puelles L (2005) Postulated boundaries and differential fate in the developing rostral hindbrain. Brain Res Brain Res Rev 49:179-190

Azmitia EC, Gannon PJ (1986) Anatomy of the serotonergic system in the primate and sub-primate brain. Adv Neurol 43:407-468

Bang SJ, Jensen P, Dymecki SM, Commons KG (2012) Projections and interconnections of genetically defined serotonin neurons in mice. Eur J Neurosci 35:85-96

Bechterew W (1899) Die Leitungsbahnen im Gehirn und Rückenmark. Georgi, Leipzig

Beitz AJ (1982) The nuclei of origin of brainstem serotonergic projections to the rodent spinal trigeminal nucleus. Neurosci Lett 32:223-228

Bjarkam CR, Sørensen JC, Geneser FA (1997) Distribution and morphology of serotonin-immunoreactive neurons in the brainstem of the New Zealand white rabbit. J Comp Neurol 380:507-519

Bobillier P, Seguin S, Petitjean F, Salvert D, Touret M, Jouvet M (1976) The raphe nuclei of the cat brain stem: a topographical atlas of their efferent projections as revealed by autoradiography. Brain Res 113:449-486

Boschi G, Desiles M, Reny V, Rips R, Wrigglesworth S (1983) Antinociceptive properties of thyrotropin releasing hormone in mice: comparison with morphine. Br J Pharmacol 79:85-92

Bowker RM, Westlund KN, Sullivan MC, Coulter JD (1982) Organization of descending serotonergic projections to the spinal cord. Prog Brain Res 57:239-265

Briscoe J, Sussel L, Serup P, Hartigan-O'Connor D, Jessell TM, Rubenstein JL, Ericson J (1999) Homeobox gene $N k x 2.2$ and specification of neuronal identity by graded Sonic hedgehog signalling. Nature 398:622-627

Brodski C, Weisenhorn DM, Signore M, Sillaber I, Oesterheld M, Broccoli V, Acampora D, Simeone A, Wurst W (2003) Location and size of dopaminergic and serotonergic cell populations are controlled by the position of the midbrain-hindbrain organizer. J Neurosci 23:4199-4207

Brown JO (1943) The nuclear pattern of the non-tectal portions of the midbrain and isthmus in the dog and cat. J Comp Neurol 78:365-405

Büttner-Enever JA, Cohen B, Pause M, Fries W (1998) Raphe nucleus of the pons containing omnipause neurons of the oculomotor system in the monkey, and its homologue in man. J Comp Neurol 267:307-321

Cambronero F (1999) Aportaciones a la organización estructural del rombencéfalo de pollo. Dissertation. University of Murcia, Spain

Cambronero F, Puelles L (2000) Rostrocaudal nuclear relationships in the avian medulla oblongata: a fate map with quail-chick chimeras. J Comp Neurol 427:522-545

Carpenter EM, Goddard JM, Chisaka O, Manley NR, Capecchi MR (1993) Loss of Hox-A1 (Hox-1.6) function results in the reorganization of the murine hindbrain. Development 118:1063-1075

Castaldi L (1923) Studi sulla struttura e sullo sviluppo del mesencefalo. Richerche in Cavia cobaya. Parte prima. Arch Ital Anat Embriol 21:172-263

Cheng L, Chen CL, Luo P, Tan M, Qiu M, Johnson R, Ma Q (2003) Lmx 1b, Pet1, and $N k x 2.2$ coordinately specify serotonergic neurotransmitter phenotype. J Neurosci 23:9961-9967

Commons KG, Valentino RJ (2002) Cellular basis for the effects of substance $\mathrm{P}$ in the periaqueductal gray and dorsal raphe nucleus. J Comp Neurol 447:82-97

Craven SE, Lim KC, Ye W, Engel JD, de Sauvage F, Rosenthal A (2004) Gata2 specifies serotonergic neurons downstream of sonic hedgehog. Development 131:1165-1173

Dahlström A, Fuxe K (1964) Evidence for the existence of monoamine-containing neurons in the central nervous system. Acta Physiol Scand Suppl 232:1-55

Daszuta A, Portalier P (1985) Distribution and quantification of 5-HT nerve cell bodies in the nucleus raphe dorsalis area of C57BL and BALBc mice. Relationship between anatomy and biochemistry. Brain Res 360:58-64

De Grandis E, Serrano M, Pérez-Dueñas B, Ormazábal A, Montero R, Alonso A, Veneselli E, Pineda M, González V, Sanmartí F, Fons C, Sans A, Cormand B, Puelles L, Campistol J, Artuch R, García-Cazorla A (2010) Cerebrospinal fluid alterations of the serotonin product 5-hydroxyindolacetic acid in neurological disorders. J Inherit Metab Dis 33:803-809

Del Fiacco M, Dessi ML, Levanti MC (1984) Topographical localization of substance $\mathrm{P}$ in the human post-mortem brainstem. An immunohistochemical study in the newborn and adult tissue. Neuroscience 12:591-611 
Deneris ES, Wyler SC (2012) Serotonergic transcriptional networks and potential importance to mental health. Nat Neurosci 15:519-527

Diaz-Cintra S, Cintra L, Kemper T, Resnick O, Morgane PJ (1981) Nucleus raphe dorsalis: a morphometric Golgi study in rats of three age groups. Brain Res 221:243-255

Díez del Corral R, Storey KG (2004) Opposing FGF and retinoid pathways: a signalling switch that controls differentiation and patterning onset in the extending vertebrate body axis. BioEssays 26:857-869

Ding YQ, Marklund U, Yuan W, Yin J, Wegman L, Ericson J, Deneris E, Johnson RL, Chen ZF (2003) Lmxlb is essential for the development of serotonergic neurons. Nat Neurosci 6:933-938

Dong HW, The Allen Institute for Brain Science (2008) The Allen reference atlas: a digital color brain atlas of the C57BL/6J male mouse. Wiley-Blackwell, Hoboken

Eaton MJ, Gudehithlu KP, Quach T, Silvia CP, Hadji-Constantinou M, Neff NH (1993) Distribution of aromatic L-amino acid decarboxylase mRNA in mouse brain by in situ hybridization histology. J Comp Neurol 337:640-654

Eiberger B, Schilling K (2012) Cerebellins: capstones to bridge the synaptic cleft. J Neurochem 121:697-699

Ellenberger HH, Feldman JL (1990) Subnuclear organization of the lateral tegmental field of the rat. I. Nucleus ambiguus and ventral respiratory group. J Comp Neurol 294:202-211

Espallergues J, Teegarden SL, Veerakumar A, Boulden J, Challis C, Jochems J, Chan M, Petersen T, Deneris E, Matthias P, Hahn CG, Lucki I, Beck SG, Berton O (2012) HDAC6 regulates glucocorticoid receptor signalling in serotonin pathways with critical impact on stress resilience. J Neurosci 32:4400-4416

Felten DL, Sladek JR (1983) Monoamine distribution in primate brain. V. Monoaminergic nuclei: anatomy, pathways, and local organization. Brain Res Bull 10:171-284

Ferrán JL, de Oliveira ED, Merchán P, Sandoval JE, Sánchez-Arrones L, Martínez-de-la-Torre M, Puelles L (2009) Genoarchitectonic profile of developing nuclear groups in the chicken pretectum. J Comp Neurol 517:405-451

Ferreira R, Wai A, Shimizu R, Gillemans N, Rottier R, von Lindern M, Ohneda K, Grosveld F, Yamamoto M, Philipsen S (2007) Dynamic regulation of Gata factor levels is more important than their identity. Blood 109:5481-5490

Fortune T, Lurie DI (2009) Chronic low-level lead exposure affects the monoaminergic system in the mouse superior olivary complex. J Comp Neurol 513:542-558

Fox SR, Deneris ES (2012) Engrailed is required in maturing serotonin neurons to regulate the cytoarchitecture and survival of the dorsal raphe nucleus. J Neurosci 32:7832-7842

Fu W, Le Maître E, Fabre V, Bernard JF, Xu ZQD (2010) Chemical neuroanatomy of the dorsal raphe nucleus and adjacent structures of the mouse brain. J Comp Neurol 518:3464-3494

Fuxe K, Hökfelt T, Olson L, Ungerstedt U (1977) Central monoaminergic pathways, with emphasis on their relation to the so called 'extrapyramidal motor system'. Pharmacol Ther B 3:169-210

Garcia-Alloza M, Zaldua N, Diez-Ariza M, Marcos B, Lasheras B, Javier Gil-Bea F, Ramirez MJ (2006) Effect of selective cholinergic denervation on the serotonergic system: implications for learning and memory. J Neuropathol Exp Neurol 65:10741081

Gaskell WH (1889) On the relations between the structure, function and origin of the cranial nerves, together with a theory on the origin of the nervous system of vertebrates. J Physiol 10: $153-211$

Gavalas A, Davenne M, Lumsden A, Chambon P, Rijli FM (1997) Role of Hoxa-2 in axon pathfinding and rostral hindbrain patterning. Development 124:3693-3702
Goddard JM, Rossel M, Manley NR, Capecchi MR (1996) Mice with targeted disruption of Hoxb-1 fail to form the motor nucleus of the VIIth nerve. Development 122:3217-3228

Goto M, Sano Y (1984) Ontogenesis of the central serotonin neuron system of the rat. An immunocytochemical study. Neurosci Res $1: 3-18$

Hale MW, Lowry CA (2011) Functional topography of midbrain and pontine serotonergic systems: implications for synaptic regulation of serotonergic circuits. Psychopharmacology 213:243-264

Halliday GM, Li YW, Joh TH, Cotton RG, Howe PR, Geffen LB, Blessing WW (1988) Distribution of substance P-like immunoreactive neurons in the human medulla oblongata: co-localization with monoamine-synthesizing neurons. Synapse 2:353-370

Hallonet M, Alvarado-Mallart RM (1997) The chick/quail chimeric system: a model for early cerebellar development. Perspect Dev Neurobiol 5:17-31

Hansson SR, Mezey E, Hoffman BJ (1998) Serotonin transporter messenger RNA in the developing rat brain: early expression in serotonergic neurons and transient expression in non-serotonergic neurons. Neuroscience 83:1185-1201

Harding A, Paxinos G, Halliday G (2004) The serotonin and tachykinin systems. In: Paxinos G (ed) The rat nervous system, 3rd edn. Elsevier Academic Press, San Diego, pp 1203-1256

Harkmark W (1954) Cell migration from the rhombic lip to the inferior olive, the nucleus raphe and the pons. A morphological and experimental investigation on chick embryos. J Comp Neurol 100:115-211

Harrison PJ, Lyon L, Sartorius LJ, Burnet PW, Lane TA (2008) The group II metabotropic glutamate receptor 3 (mGluR3, mGlu3, GRM3): expression, function and involvement in schizophrenia. J Psychopharmacol 22:308-322

Hawthorne AL, Wylie CJ, Landmesser LT, Deneris ES, Silver J (2010) Serotonergic neurons migrate radially through the neuroepithelium by dynamin-mediated somal translocation. J Neurosci 30:420-430

Hendricks T, Francis N, Fyodorov D, Deneris ES (1999) The ETS domain factor Pet-1 is an early and precise marker of central serotonin neurons and interacts with a conserved element in serotonergic genes. J Neurosci 19:10348-10356

Hendricks TJ, Fyodorov DV, Wegman LJ, Lelutiu NB, Pehek EA, Yamamoto B, Silver J, Weeber EJ, Sweatt JD, Deneris ES (2003) Pet-1 ETS gene plays a critical role in 5-HT neuron development and is required for normal anxiety-like and aggressive behavior. Neuron 37:233-247

Hermann GE, Holmes GM, Rogers RC, Beattie MS, Bresnahan JC (2003) Descending spinal projections from the rostral gigantocellular reticular nuclei complex. J Comp Neurol 455:210-221

Hidalgo-Sánchez M, Millet S, Simeone A, Alvarado-Mallart RM (1999) Comparative analysis of Otx2, Gbx2, Pax2, Fgf8 and Wnt1 gene expressions during the formation of the chick midbrain/hindbrain domain. Mech Dev 81:175-178

Hidalgo-Sánchez M, Martínez-de-la-Torre M, Alvarado-Mallart RM, Puelles L (2005) Distinct pre-isthmic domain, defined by overlap of Otx2 and Pax2 expression domains in the chicken caudal midbrain. J Comp Neurol 483:17-29

His W (1893) Vorschläge zur Eintheilung des Gehirns. Arch Anat EntwGesch 1893:173-179

His W (1895) Die Anatomische Nomenclatur. Nomina Anatomica. Neurologie. Arch Anat EntwGesch Suppl Bd 1895:155-177

Hoffman BJ, Hansson SR, Mezey E, Palkovits M (1998) Localization and dynamic regulation of biogenic amine transporters in the mammalian central nervous system. Front Neuroendocrinol 19:187-231

Holstege JC, de Graaff W, Hossaini M, Cano SC, Jaarsma D, Akker EV, Deschamps J (2008) Loss of Hoxb8 alters spinal dorsal laminae and sensory responses in mice. Proc Natl Acad Sci USA 105:6338-6343 
Hornung JP (2003) The human raphe nuclei and the serotonergic system. J Chem Neuroanat 26:331-343

Hornung JP, Fritschy JM (1988) Serotonergic system in the brainstem of the marmoset: a combined immunocytochemical and threedimensional reconstruction study. J Comp Neurol 270:471-487

Hylden JL, Hayashi H, Ruda MA, Dubner R (1986) Serotonin innervation of physiologically identified lamina I projection neurons. Brain Res 370:401-404

Imai H, Steindler DA, Kitai ST (1986) The organization of divergent axonal projections from the midbrain raphe nuclei in the rat. J Comp Neurol 243:363-380

Irving C, Mason I (2000) Signalling by FGF8 from the isthmus patterns anterior hindbrain and establishes the anterior limit of Hox gene expression. Development 127:177-186

Ishimura K, Takeuchi Y, Fujiwara K, Tominaga M, Yoshioka H, Sawada T (1988) Quantitative analysis of the distribution of serotonin-immunoreactive cell bodies in the mouse brain. Neurosci Lett 91:265-270

Jacob J, Ferri AL, Milton C, Prin F, Pla P, Lin W, Gavalas A, Ang SL, Briscoe J (2007) Transcriptional repression coordinates the temporal switch from motor to serotonergic neurogenesis. Nat Neurosci 10:1433-1439

Jacobs BL, Azmitia EC (1992) Structure and function of the brain serotonin system. Physiol Rev 72:165-229

Jacobs BL, Gannon PJ, Azmitia EC (1984) Atlas of serotonergic cell bodies in the cat brainstem: an immunocytochemical analysis. Brain Res Bull 13:1-31

Jensen P, Anna F, Farago AF, Awatramani RB, Scott MM, Deneris ES, Dymecki SM (2008) Redefining the serotonergic system by genetic lineage. Nature Neurosci 11:417-419

Johnston JB (1902) An attempt to define the primitive functional divisions of the central nervous system. J Comp Neurol 12:87-106

Joyner AL, Liu A, Millet S (2000) Otx2, Gbx2 and Fgf8 interact to position and maintain a midbrain-hindbrain organizer. Curr Opin Cell Biol 12:736-741

Kala K, Jukkola T, Pata I, Partanen J (2008) Analysis of the midbrainhindbrain boundary cell fate using a boundary cell-specific Cremouse strain. Genesis 46:29-36

Kirifides ML, Simpson KL, Lin RC, Waterhouse BD (2001) Topographic organization and neurochemical identity of dorsal raphe neurons that project to the trigeminal somatosensory pathway in the rat. J Comp Neurol 435:325-340

Köhler C, Steinbusch H (1982) Identification of serotonin and nonserotonin-containing neurons of the midbrain raphe projecting to the entorhinal area and the hippocampal formation. A combined immunohistochemical and fluorescent retrograde tracing study in the rat brain. Neuroscience 7:951-975

Kriegebaum C, Song NN, Gutknecht L, Huang Y, Schmitt A, Reif A, Ding YQ, Lesch KP (2010) Brain-specific conditional and timespecific inducible Tph2 knockout mice possess normal serotonergic gene expression in the absence of serotonin during adult life. Neurochem Int 57:512-517

Kuhlenbeck H (1973) The central nervous system of vertebrates, Vol 3, part II: Overall morphological pattern. Karger, Basel

Lee SB, Lee HS, Waterhouse BD (2008) The collateral projection from the dorsal raphe nucleus to whisker-related, trigeminal sensory and facial motor systems in the rat. Brain Res 1214:11-22

Léger L, Gay N, Cespuglio R (2002) Neurokinin NK1- and NK3immunoreactive neurons in serotonergic cell groups in the rat brain. Neurosci Lett 323:146-150

Lidov HGW, Molliver ME (1982) Immunohistochemical study of the development of serotonergic neurons in the rat CNS. Brain Res Bull 9:559-604

Liu C, Maejima T, Wyler SC, Casadesus G, Herlitze S, Deneris ES (2010) Pet-1 is required across different stages of life to regulate serotonergic function. Nat Neurosci 13:1190-1198
Lorente-Cánovas B, Marín F, Corral-San-Miguel R, Hidalgo-Sánchez M, Ferrán JL, Puelles L, Aroca P (2012) Multiple origins, migratory paths and molecular profiles of cells populating the avian interpeduncular nucleus. Dev Biol 361:12-26

Lumsden A, Krumlauf R (1996) Patterning the vertebrate neuraxis. Science 274:1109-1115

Mamounas LA, Mullen CA, O'Hearn E, Molliver ME (1991) Dual serotoninergic projections to forebrain in the rat: morphologically distinct 5-HT axon terminals exhibit differential vulnerability to neurotoxic amphetamine derivatives. J Comp Neurol 314:558-586

Marburg O (1910) Mikroskopisch-topographischer Atlas des menschlichen Zentralnervensystems. Franz Deuticke, Leipzig

Marín F, Puelles L (1995) Morphological fate of rhombomeres in quail/chick chimeras: a segmental analysis of hindbrain nuclei. Eur J Neurosci 7:1714-1738

Marín F, Aroca P, Puelles L (2008) Hox gene co-linear expression in the avian medulla oblongata is correlated with pseudorhombomeric domains. Dev Biol 323:230-247

Martinez S, Alvarado-Mallart RM (1989) Rostral cerebellum originates from the caudal portion of the so-called 'mesencephalic' vesicle: a study using chick/quail chimeras. Eur J Neurosci 1:549-560

Martínez S, Puelles E, Puelles L, Echevarria D (2012) Molecular regionalization of the developing neural tube. In: Watson $\mathrm{C}$, Paxinos G, Puelles L (eds) The mouse nervous system. Elsevier Academic Press, San Diego, pp 2-18

Meessen H, Olszewski J (1949) A cytoarchitectonic atlas of the rhombencephalon of the rabbit. Karger, Basel

Merchenthaler I (1984) Corticotropin releasing factor (CRF)-like immunoreactivity in the rat central nervous system. Extrahypothalamic distribution. Peptides 5(suppl 1):53-69

Meyer-Bernstein EL, Morin LP (1996) Differential serotonergic innervation of the suprachiasmatic nucleus and the intergeniculate leaflet, and its role in circadian rhythm modulation. J Neurosci 16:2097-2111

Moens CB, Prince VE (2002) Constructing the hindbrain: insights from the zebrafish. Dev Dyn 224:1-17

Molliver ME (1987) Serotonergic neuronal systems: what their anatomic organization tells us about function. J Clin Psychopharmacol 7(suppl 6):3S-23S

Morillo AM, Nuñez-Abades PA, Gaytan SP, Pasaro R (1995) Brain stem projections by axonal collaterals to the rostral and caudal ventral respiratory group in the rat. Brain Res Bull 37:205-211

Nardelli J, Thiesson D, Fujiwara Y, Tsai FY, Orkin SH (1999) Expression and genetic interaction of transcription factors Gata2 and Gata-3 during development of the mouse central nervous system. Dev Biol 210:305-321

Nieuwenhuys R (1985) The chemoarchitecture of the brain. Springer, Heidelberg

Nieuwenhuys R (1998) Comparative neuroanatomy: Place, principles and programme. In: Nieuwenhuys R, ten Donkelaar HJ, Nicholson C (eds) The central nervous system of vertebrates, vol 1. Springer, Berlin, pp 273-326

Nieuwenhuys R (2001) The brainstem of mice, men and other mammals. Nature Rev Neurosci. doi:10.1038/nrn0901_611a-c1

Nieuwenhuys R (2009) Analysis of the structure of the brain stem of mammals by means of a modified D'Arcy Thompson procedure. Brain Struct Funct 214:79-85

Nieuwenhuys R (2011) The structural, functional, and molecular organization of the brainstem. Front Neuroanat 5:33

Nieuwenhuys R, Voogd J, van Huijzen C (2008) The human central nervous system, 4th edn. Springer, Berlin

Nillni EA, Sevarino KA (1999) The biology of pro-thyrotropinreleasing hormone-derived peptides. Endocr Rev 20:599-648 
O'Hearn E, Molliver ME (1984) Organization of raphe-cortical projections in rat: a quantitative retrograde study. Brain Res Bull 13:709-726

Olson L, Seiger A (1972) Early prenatal ontogeny of central monoamine neurons in the rat: fluorescence histochemical observations. Z Anat Entwickl Gesch 137:301-316

Olszewski J, Baxter D (1954) Cytoarchitecture of the human brain stem. Karger, Basel

Orr H (1887) Contribution to the embryology of the lizard. J Morphol $1: 311-372$

Oury F, Murakami Y, Renaud JS, Pasqualetti M, Charnay P, Ren SY, Rijli FM (2006) Hoxa2- and rhombomere-dependent development of the mouse facial somatosensory map. Science 313:1408-1413

Päällysaho J, Sugita S, Noda H (1991) Brainstem mossy fiber projections to lobules VIa, VIb, c, VII and VIII of the cerebellar vermis in the rat. Neurosci Res 12:217-231

Palmgren A (1921) Embryological and morphological studies on the midbrain and cerebellum of vertebrates. Acta Zool (Stockholm) 2:1-94

Pasquier DA, Villar MJ (1982) Specific serotonergic projections to the lateral geniculate body from the lateral cell groups of the dorsal raphe nucleus. Brain Res 249:142-146

Pattyn A, Vallstedt A, Dias JM, Samad OA, Krumlauf R, Rijli FM, Brunet JF, Ericson J (2003) Coordinated temporal and spatial control of motor neuron and serotonergic neuron generation from a common pool of CNS progenitors. Genes Dev 17:729-737

Paxinos G, Franklin KBJ (2007) The mouse brain in stereotaxic coordinates, 3rd edn. Elsevier Academic Press, San Diego

Paxinos G, Watson C (2007) The rat brain in stereotaxic coordinates, 6th edn. Elsevier Academic Press, San Diego

Pfaar H, von Holst A, Vogt Weisenhorn DM, Brodski C, Guimera J, Wurst W (2002) mPet-1, a mouse ETS-domain transcription factor, is expressed in central serotonergic neurons. Dev Genes Evol 212:43-46

Porreca F, Ossipov MH, Gebhart GF (2002) Chronic pain and medullary descending facilitation. Trends Neurosci 25:319-325

Poulat P, Marlier L, Rajaofetra N, Privat A (1992) 5-Hydroxytryptamine, substance $\mathrm{P}$ and thyrotropin-releasing hormone synapses in the intermediolateral cell column of the rat thoracic spinal cord. Neurosci Lett 136:19-22

Priestley JV, Wotherspoon G, Savery D, Averill S, Rattray M (1993) A combined in situ hybridization and immunofluorescence procedure allowing visualization of peptide mRNA and serotonin in single sections. J Neurosci Methods 48:99-110

Puelles L (2009a) Forebrain development: Prosomeric model. In: Lemke G (ed) Developmental neurobiology. Elsevier Academic Press, San Diego, pp 95-99

Puelles L (2009b) Forebrain development: Prosomeric model. In: Squire LR (ed) Encyclopedia of neuroscience. Academic Press, London, pp 315-319

Puelles L (2012) Plan of the developing vertebrate nervous system: relating embryology to the adult nervous system (prosomere model, overview of brain organization). In: Rubenstein J, Rakic $P$ (eds) Developmental neuroscience: a comprehensive reference. Elsevier Academic Press, San Diego (in press)

Puelles L, Rubenstein JL (2003) Forebrain gene expression domains and the evolving prosomeric model. Trends Neurosci 26:469-476

Puelles E, Annino A, Tuorto F, Usiello A, Acampora D, Czerny T, Brodski C, Ang SL, Wurst W, Simeone A (2004) Otx2 regulates the extent, identity and fate of neuronal progenitor domains in the ventral midbrain. Development 131:2037-2048

Puelles L, Martinez-de-la-Torre M, Paxinos G, Watson C, Martinez S (2007) The chick brain in stereotaxic coordinates. An atlas featuring neuromeric subdivisions and mammalian homologies. Elsevier Academic Press, San Diego

Puelles L, Martínez-de-la-Torre M, Bardet S, Rubenstein JLR (2012a) Hypothalamus. In: Watson C, Paxinos G, Puelles L (eds) The mouse nervous system. Academic Press-Elsevier, San Diego, pp 221-312

Puelles E, Martínez-de-la-Torre M, Watson C, Puelles L (2012b) Midbrain. In: Watson C, Paxinos G, Puelles L (eds) The mouse nervous system. Academic Press-Elsevier, San Diego, pp 338-359

Ramón y Cajal S (1909) Histologie du system nerveux de l'homme et des vertebrés, vol II (re-edition) CSIC, Madrid

Rikard-Bell GC, Törk I, Sullivan C, Scheibner T (1990) Distribution of substance P-like immunoreactive fibers and terminals in the medulla oblongata of the human infant. Neuroscience 34:133-148

Rodrigues SL, Maseko BC, Ihunwo AO, Fuxe K, Manger PR (2008) Nuclear organization and morphology of serotonergic neurons in the brain of the Nile crocodile, Crocodylus niloticus. J Chem Neuroanat 35:133-145

Rodrigues BC, Cavalcante JC, Elias CF (2011) Expression of cocaine- and amphetamine-regulated transcript in the rat forebrain during postnatal development. Neuroscience 195:201-214

Rodríguez JJ, Noristani HN, Hoover WB, Linley SB, Vertes RP (2011) Serotonergic projections and serotonin receptor expression in the reticular nucleus of the thalamus in the rat. Synapse 65:919-928

Rubenstein JLR, Puelles L (1992) Homeobox gene expression during development of the vertebrate brain. In: Pedersen RA (ed) Current topics in developmental biology. Academic Press, San Diego, pp 1-63

Ruda MA, Coffield J, Steinbusch WM (1982) Immunocytochemical analysis of serotonergic axons in laminae I y II of the lumbar spinal cord of the cat. J Neurosci 2:1660-1671

Sakanaka M, Shibasaki T, Lederis K (1987) Corticotropin releasing factor-like immunoreactivity in the rat brain as revealed by a modified cobalt-glucose oxidase-diaminobenzidine method. J Comp Neurol 260:256-298

Sako H, Kojima T, Okado N (1986) Immunohistochemical study on the development of serotoninergic neurons in the chick: I. Distribution of cell bodies and fibers in the brain. J Comp Neurol 253:61-78

Sasek CA, Wessendorf MW, Helke CJ (1990) Evidence for coexistence of thyrotropin-releasing hormone, substance $\mathrm{P}$ and serotonin in ventral medullary neurons that project to the intermediolateral cell column in the rat. Neuroscience 35:105-119

Schneider-Maunoury S, Gilardi-Hebenstreit P, Charnay P (1998) How to build a vertebrate hindbrain. Lessons from genetics. C R Acad Sci III 321:819-834

Seiger A, Olson L (1973) Late prenatal ontogeny of central monoamine neurons in the rat: fluorescence histochemical observations. Z Anat Entwickl Gesch 140:281-318

Shimamura K, Hirano S, McMahon AP, Takeichi M (1994) Wnt-1dependent regulation of local E-cadherin and alpha N-catenin expression in the embryonic mouse brain. Development 120:2225-2234

Shimamura K, Hartigan DJ, Martínez S, Puelles L, Rubenstein JLR (1995) Longitudinal organization of the anterior neural plate and neural tube. Development 121:3923-3933

Simeone A (2000) Positioning the isthmic organizer where Otx2 and Gbx2 meet. Trends Genet 16:237-240

Simeone A, Acampora D, Gulisano M, Stornaiuolo A, Boncinelli E (1992) Nested expression domains of four homeobox genes in developing rostral brain. Nature 358:687-690 
Simeone A, D'Apice MR, Nigro V, Casanova J, Graziani F, Acampora D, Avantaggiato V (1994) Orthopedia, a novel homeobox-containing gene expressed in the developing CNS of both mouse and Drosophila. Neuron 13:83-101

Simeone A, Puelles E, Omodei D, Acampora D, Di Giovannantonio LG, Di Salvio M, Mancuso P, Tomasetti C (2011) Otx genes in neurogenesis of mesencephalic dopaminergic neurons. Dev Neurobiol 71:665-679

Simon HH, Scholz C, O'Leary DDM (2005) Engrailed genes control developmental fate of serotonergic and noradrenergic neurons in mid- and hindbrain in a gene dose-dependent manner. Mol Cell Neurosci 28:96-105

Skagerberg G, Björklund A (1985) Topographic principles in the spinal projections of serotonergic and non-serotonergic brainstem neurons in the rat. Neuroscience 15:445-480

Smidt MD, Asbreuk CH, Cox JJ, Chen H, Johnson RL, Burbach JP (2000) A second independent pathway for development of mesencephalic dopaminergic neurons requires Lmxlb. Nat Neurosci 3:337-341

Smith GS, Savery D, Marden C, Lopez Costa JJ, Averill S, Priestley JV, Rattray M (1994) Distribution of messenger RNAs encoding enkephalin, substance $\mathrm{P}$, somatostatin, galanin, vasoactive intestinal polypeptide, neuropeptide $\mathrm{Y}$, and calcitonin generelated peptide in the midbrain periaqueductal grey in the rat. J Comp Neurol 350:23-40

Soiza-Reilly M, Commons KG (2011) Glutamatergic drive of the dorsal raphe nucleus. J Chem Neuroanat 41:247-255

Song NN, Xiu JB, Huang Y, Chen JY, Zhang L, Gutknecht L, Lesch KP, Li H, Ding YQ (2011) Adult raphe-specific deletion of Lmxlb leads to central serotonin deficiency. PLoS One 6:e15998. doi:10.1371/journal.pone.0015998

Steinbusch HW (1981) Distribution of serotonin-immunoreactivity in the central nervous system of the rat. Cell bodies and terminals. Neuroscience 6:557-618

Steinbusch HWM, Nieuwenhuys R (1983) The raphe nuclei of the rat brainstem: a cytoarchitectonic and immunohistochemical study. In: Emson PC (ed) Chemical neuroanatomy. Raven Press, New York, pp 131-207

Stratford TR, Wirtshafter D (1988) Evidence for a projection from the B9 serotonergic cell group to the median raphe nucleus. Brain Res Bull 21:325-328

Studer M, Lumsden A, Ariza-McNaughton L, Bradley A, Krumlauf R (1996) Altered segmental identity and abnormal migration of motor neurons in mice lacking Hoxb-1. Nature 384:630-634

Swanson LW (1992) Brain maps. Structure of the rat brain, Elsevier, Amsterdam

Swanson LW (1993) The central nervous system: major divisions and cell groups. Trends in Neurosci (separate folded map in the November 1993 number)

Swanson LW (1998) Brain maps: structure of the rat brain. 2nd rev edn., Elsevier, Amsterdam

Swanson LW (2003) Brain architecture. Oxford Univ Press, Oxford/ New York

Taber E, Brodal A, Walberg F (1960) The raphe nuclei of the brain stem in the cat I. Normal topography and cytoarchitecture and general discussion. J Comp Neurol 114:161-187

Tan K, LeDouarin NM (1991) Development of the nuclei and cell migration in the medulla oblongata. Application of the quailchick chimera system. Anat Embryol 183:321-343

Theiler K (1989) The house mouse: atlas of embryonic development. Springer, New York

Törk I (1990) Anatomy of the serotonergic system. Ann N Y Acad Sci 600:9-34

Törk I, Hornung JP (1990) Raphe nuclei and the serotonergic system. In: Paxinos G, Mai JK (eds) The human nervous system. Elsevier Academic Press, San Diego, pp 1001-1021
Tümpel S, Wiedemann LM, Krumlauf R (2009) Hox genes and segmentation of the vertebrate hindbrain. Curr Top Dev Biol $88: 103-137$

Ugrumov MV (1997) Hypothalamic monoaminergic systems in ontogenesis: development and functional significance. Int J Dev Biol 41:809-816

Ugrumov MV, Taxi J, Steinbusch HW, Tramu G, Mitskevich MS (1989) On the distribution and morpho-functional characteristics of 5-HT-immunoreactive cells in the hypothalamus of fetuses and neonatal rats. Brain Res Dev Brain Res 46:233-241

Vaage S (1969) The segmentation of the primitive neural tube in chick embryos (Gallus domesticus). A morphological, histochemical and autoradiographical investigation. Ergeb Anat Entwicklungsgesch 41:3-87

Vaage S (1973) The histogenesis of the isthmic nuclei in chick embryos (Gallus domesticus). I. A morphological study. Z Anat Entwicklungsgesch 142:283-314

Valverde F (1962) Reticular formation of the albino rat's brain stem cytoarchitecture and corticofugal connections. J Comp Neurol 119:25-53

van der Kooy D, Hunt SP, Steinbusch HW, Verhofstad AA (1981) Separate populations of cholecystokinin and 5-hydroxytryptamine-containing neuronal cells in the rat dorsal raphe, and their contribution to the ascending raphe projections. Neurosci Lett 26:25-30

van Doorninck JH, van Der Wees J, Karis A, Goedknegt E, Engel JD, Coesmans M, Rutterman M, Grosveld F, De Zeeuw CI (1999) Gata-3 is involved in the development of serotonergic neurons in the caudal raphe nuclei. J Neurosci 19:RC12

Vanderhaeghen JJ (1985) Neuronal cholecystokinin. In: Björklund A, Hökfelt T (eds) Handbook of chemical neuroanatomy, vol 4., GABA and neuropeptides in the CNS, part 1Elsevier, Amsterdam, pp 406-435

Veasey SC, Fornal CA, Metzler CW, Jacobs BL (1995) Response of serotonergic caudal raphe neurons in relation to specific motor activities in freely moving cats. J Neurosci 15:5346-5359

Vertes RP (1991) A PHA-L analysis of ascending projections of the dorsal raphe nucleus in the rat. J Comp Neurol 313:643-668

Vertes RP, Crane AM (1997) Distribution, quantification, and morphological characteristics of serotonin-immunoreactive cells of the supralemniscal nucleus (B9) and pontomesencephalic reticular formation in the rat. J Comp Neurol 378:411-424

Vertes RP, Martin GF (1988) Autoradiographic analysis of ascending projections from the pontine and mesencephalic reticular formation and the median raphe nucleus in the rat. J Comp Neurol 275:511-541

Vertes RP, Fortin WJ, Crane AM (1999) Projections of the median raphe nucleus in the rat. J Comp Neurol 407:555-582

Villar MJ, Vitale ML, Hökfelt T, Verhofstad AA (1988) Dorsal raphe serotoninergic branching neurons projecting both to the lateral geniculate body and superior colliculus: a combined retrograde tracing-immunohistochemical study in the rat. J Comp Neurol 277:126-140

Wallace JA, Lauder JM (1983) Development of the serotonergic system in the rat embryo: an immunocytochemical study. Brain Res Bull 10:459-479

Waselus M, Galvez JP, Valentino RJ, Van Bockstaele EJ (2006) Differential projections of dorsal raphe nucleus neurons to the lateral septum and striatum. J Chem Neuroanat 31:233-242

Waterhouse BD, Border B, Wahl L, Mihailoff GA (1993) Topographic organization of rat locus coeruleus and dorsal raphe nuclei: distribution of cells projecting to visual system structures. J Comp Neurol 336:345-361

Watson C (2012) Hindbrain. In: Watson C, Paxinos G, Puelles L (eds) The mouse nervous system. Elsevier Academic Press, San Diego, pp 398-423 
Watson C, Paxinos G (2010) Chemoarchitectonic atlas of the mouse brain. Elsevier Academic Press, San Diego

Watson C, Kirkcaldie M, Paxinos G (2010) The brain. An introduction to functional neuroanatomy. Elsevier Academic Press, San Diego

Weilan Y, Shimamura K, Rubenstein JLR, Hynes MA, Rosenthal A (1998) Fgf and Shh signals control dopaminergic and serotonergic cell fate in the anterior neural plate. Cell 93:755-766

Willoughby JO, Blessing WW (1987) Origin of serotonin innervation of the arcuate and ventromedial hypothalamic region. Brain Res 418:170-173

Wingate RJ, Lumsden A (1996) Persistence of rhombomeric organization in the postsegmental hindbrain. Development 122:2143-2152

Winkler C, Potter A (1914) An anatomical guide to experimental researches on the cat's brain. A series of 35 frontal sections. W Versluys, Amsterdam

Wolfer DP, Henehan-Beatty A, Stoeckli ET, Sonderegger P, Lipp HP (1994) Distribution of TAG-1/axonin-1 in fiber tracts and migratory streams of the developing mouse nervous system. J Comp Neurol 345:1-32

Wu W, Elde R, Wessendorf MW (1993) Organization of the serotonergic innervation of spinal neurons in rats. III. Differential serotonergic innervation of somatic and parasympathetic preganglionic motoneurons as determined by patterns of coexisting peptides. Neuroscience 55:223-233

Wylie CJ, Hendricks TJ, Zhang B, Wang L, Lu P, Leahy P, Fox S, Maeno H, Deneris ES (2010) Distinct transcriptomes define rostral and caudal serotonin neurons. J Neurosci 30:670-684

Zervas M, Millet S, Ahn S, Joyner AL (2004) Cell behaviors and genetic lineages of the mesencephalon and rhombomere 1. Neuron 43:345-357

Zhao ZQ, Scott M, Chiechio S, Wang JS, Renner KJ, Gereau RW, Johnson RL, Deneris ES, Chen ZF (2006) Lmxlb is required for maintenance of central serotonergic neurons and mice lacking central serotonergic system exhibit normal locomotor activity. J Neurosci 26:12781-12788 